\title{
Teoria isomorfa dos espaços de Banach
}

$C_{0}(K, X)$

Leandro Candido Batista

\author{
TESE APRESENTADA \\ $\mathrm{AO}$ \\ Instituto de Matemática e Estatística \\ DA \\ Universidade DE SÃo PAUlo \\ PARA \\ OBTENÇÃO DO TÍTULO \\ $\mathrm{DE}$ \\ DOUTOR EM CIÊNCIAS
}

Programa: Matemática

Orientador: Prof. Dr. Elói Medina Galego

Durante o desenvolvimento deste trabalho o autor recebeu auxílio financeiro do CNPq (processo 142423/2011-4) e da CAPES

São Paulo, Novembro de 2012 


\section{Teoria isomorfa dos espaços de Banach \\ $C_{0}(K, X)$}

Esta tese contém as correções e alterações sugeridas pela Comissão Julgadora durante a defesa realizada por Leandro Candido Batista em 12/11/2012.

O original encontra-se disponível no Instituto de Matemática e Estatística da Universidade de São Paulo.

Comissão Julgadora:

- Prof. Dr. Elói Medina Galego - IME-USP

- Prof. Dr. Valentin Raphael Henri Ferenczi - IME-USP

- Prof. Dr. Jorge Tulio Mujica Ascui - UNICAMP

- Prof. Dr. Antonio Roberto da Silva - UFRJ

- Prof. Dr. Leandro Fiorini Aurichi - ICMC-USP 


\section{Agradecimentos}

Ao longo deste trabalho muitos foram os que de alguma forma me ajudaram e encorajaram a alcançar o seu término. Por essa razão, desejo expressar os meus sinceros agradecimentos:

Ao Professor Doutor Elói Medina Galego, meu orientador, pela competência científica, pela disponibilidade, pela amizade e generosidade reveladas ao longo deste período.

Aos membros da Comissão Julgadora pelas valiosas sugestões que contribuiram de forma significativa para o enriquecimento deste trabalho.

À minha amada esposa, Rita Cavalcanti, pelo inestimável apoio familiar, pela paciência e compreensão reveladas ao longo destes anos.

À minha família, meus pais Joaquim e Vera e meu irmão Leonardo, pelo enorme carinho e incentivo.

Ao Professor Doutor Daniel Victor Tausk pela valiosa ajuda sanando minhas inúmeras dúvidas sempre com brilhantismo e bom humor.

Aos Professores, Aleksander Pełczyński, Yoav Benyamini e Krzysztof Jarosz, por suas inestimáveis opiniões, sugestões e interesse pelo trabalho.

Aos grandes amigos, André Pierro de Camargo, Cesar Adriano Batista e Renato Alessandro Martins, pelo apoio.

Ao Instituto de Matemática e Estatística da Universidade de São Paulo pela oportunidade.

À Coordenação de Aperfeiçoamento de Pessoal de Nível Superior, CAPES, e ao Conselho Nacional de Desenvolvimento Científico e Tecnológico, CNPq, pelo apoio financeiro.

Mais uma vez, a todos os meus sinceros agradecimentos. 


\section{Resumo}

Para um espaço localmente compacto de Hausdorff $K$ e um espaço de Banach $X$, denotamos por $C_{0}(K, X)$ o espaço de todas as funções a valores em $X$ contínuas sobre $K$ que se anulam no infinito, munido da norma do supremo. No espírito do clássico teorema de Banach-Stone 1937, estabelecemos que se $C_{0}\left(K_{1}, X\right)$ é isomorfo a $C_{0}\left(K_{2}, X\right)$, onde $X$ é um espaço de Banach de cotipo finito e tal que $X$ é separável ou $X^{*}$ tem a propriedade de Radon-Nikodým, então ou $K_{1}$ e $K_{2}$ são ambos finitos ou $K_{1}$ e $K_{2}$ tem a mesma cardinalidade. Trata-se de uma extensão vetorial de um resultado de Cengiz 1978, o caso escalar $X=\mathbb{R}$ ou $X=\mathbb{C}$.

Demonstramos também que se $K_{1}$ e $K_{2}$ são intervalos compactos de ordinais e $X$ é um espaço de Banach de cotipo finito, então a existência de um isomorfismo $T$ de $C\left(K_{1}, X\right)$ em $C\left(K_{2}, X\right)$ com $\|T\|\left\|T^{-1}\right\|<3$ implica que uma certa soma topológica finita de $K_{1}$ é homeomorfa a alguma soma topológica finita de $K_{2}$. Mais ainda, se $X^{n}$ não contém subespaço isomorfo a $X^{n+1}$ para todo $n \in \mathbb{N}$, então $K_{1}$ é homeomorfo a $K_{2}$. Em outras palavras, obtemos um teorema tipo Banach-Stone vetorial que é uma extensão de um teorema de Gordon de 1970 e ao mesmo tempo uma extensão de um teorema de Behrends e Cambern de 1988. Mostramos que se existe um isomorfismo $T$ de $C\left(K_{1}\right)$ em um subespaço de $C\left(K_{2}, X\right)$ com $\|T\|\left\|T^{-1}\right\|<3$, então a cardinalidade do $\alpha$-ésimo derivado de $K_{2}$ ou é finita ou é maior do que a cardinalidade do $\alpha$-ésimo derivado de $K_{1}$, para todo ordinal $\alpha$.

Em seguida, seja $n$ um inteiro positivo, $\Gamma$ um conjunto infinito munido da topologia discreta e $X$ um espaço de Banach de cotipo finito. Estabelecemos que se o $n$-ésimo derivado de $K$ for não vazio, então a distância de Banach-Mazur entre $C_{0}(K, X)$ e $C_{0}(\Gamma, X)$ é maior ou igual a $2 n+1$. Também demonstramos que para quaisquer inteiros positivos $n$ e $k$, a distância de Banach-Mazur entre $C\left(\left[1, \omega^{n} k\right], X\right)$ e $C_{0}(\mathbb{N}, X)$ é exatamente $2 n+1$. Estes resultados fornecem extensões vetoriais para alguns teoremas de Cambern de 1970.

Para um ordinal enumerável $\alpha$, denotando por $C(\alpha)$ o espaço de Banach das funções contínuas no intervalo de ordinal $[1, \alpha]$, obtemos cotas superiores $H(n, k)$ e cotas inferiores $G(n, k)$ para as distâncias de Banach-Mazur entre os espaços $C(\omega)$ e $C\left(\omega^{n} k\right), 1 \leq n, k<\omega$, verificando $H(n, k)$ $G(n, k)<2$. Estas estimativas fornecem uma resposta para uma questão de Bessaga e Pełczyński de 1960 sobre as distâncias de Banach-Mazur entre $C(\omega)$ e cada um dos espaços $C(\alpha), \omega \leq \alpha<\omega^{\omega}$.

Palavras-chave: Espaços de Banach, Espaços de funções contínuas a valores vetoriais, Isomorfismos, Teorema de Banach-Stone, Distâncias de Banach-Mazur. 


\section{Abstract}

For a locally compact Hausdorff space $K$ and a Banach space $X$, we denote by $C_{0}(K, X)$ the space of $X$-valued continuous functions on $K$ which vanish at infinity, endowed with the supremum norm. In the spirit of the classical 1937 Banach-Stone theorem, we prove that if $C_{0}\left(K_{1}, X\right)$ is isomorphic to $C_{0}\left(K_{2}, X\right)$, where $X$ is a Banach space having finite cotype and such that $X$ is separable or $X^{*}$ has the Radon-Nikodým property, then either $K_{1}$ and $K_{2}$ are finite or $K_{1}$ and $K_{2}$ have the same cardinality. It is a vector-valued extension of a 1978 Cengiz result, the scalar case $X=\mathbb{R}$ or $X=\mathbb{C}$.

We also prove that if $K_{1}$ and $K_{2}$ are compact ordinal spaces and $X$ is Banach space having finite cotype, then the existence of an isomorphism $T$ from $C\left(K_{1}, X\right)$ onto $C\left(K_{2}, X\right)$ with $\|T\|\left\|T^{-1}\right\|<3$ implies that some finite topological sum of $K_{1}$ is homeomorphic to some finite topological sum of $K_{2}$. Moreover, if $X^{n}$ contains no subspace isomorphic to $X^{n+1}$ for every $n \in \mathbb{N}$, then $K_{1}$ is homeomorphic to $K_{2}$. In other words, we obtain a vector-valued Banach-Stone theorem which is an extension of a 1970 Gordon theorem and at same time an improvement of a 1988 Behrends and Cambern theorem. We show that if there is an embedding $T$ of a $C\left(K_{1}\right)$ into $C\left(K_{2}, X\right)$ with $\|T\|\left\|T^{-1}\right\|<3$, then the cardinality of the $\alpha$-th derivative of $K_{2}$ is either finite or greater than the cardinality of the $\alpha$-th derivative of $K_{1}$, for every ordinal $\alpha$.

Next, let $n$ be a positive integer, $\Gamma$ an infinite set with the discrete topology and $X$ is a Banach space having finite cotype. We prove that if the $n$-th derivative of $K$ is not empty, then the Banach Mazur distance between $C_{0}(K, X)$ and $C_{0}(\Gamma, X)$ is greater than or equal to $2 n+1$. Thus, we also show that for every positive integers $n$ and $k$, the Banach Mazur distance between $C\left(\left[1, \omega^{n} k\right], X\right)$ and $C_{0}(\mathbb{N}, X)$ is exactly $2 n+1$. These results provide vector-valued versions of some 1970 Cambern theorems.

For a countable ordinal $\alpha$, writing $C(\alpha)$ for the Banach space of continuous functions on the interval of ordinal $[1, \alpha]$, we give lower bounds $H(n, k)$ and upper bounds $G(n, k)$ on the BanachMazur distances between $C(\omega)$ and $C\left(\omega^{n} k\right), 1 \leq n, k<\omega$, such that $H(n, k)-G(n, k)<2$. These estimates provide an answer to a 1960 Bessaga and Pełczyński question on the Banach-Mazur distances between $C(\omega)$ and each of the $C(\alpha)$ spaces, $\omega \leq \alpha<\omega^{\omega}$

Keywords: Banach spaces, Spaces of vector-valued continuous functions, Isomorphisms, BanachStone Theorem, Banach-Mazur distances. 


\section{Sumário}

Lista de Símbolos $\quad$ xiii

Introdução $\quad$ xv

1 Medidas vetoriais e integração vetorial $\quad 1$

1.1 Introdução . . . . . . . . . . . . . . . . . . . . . . . . . . 1

1.2 Medidas Vetoriais . . . . . . . . . . . . . . . . . . . . . 1

1.3 Integração vetorial . . . . . . . . . . . . . . . . . . . . . . . 3

1.4 Representação de funcionais em $C_{0}(K, X) \ldots \ldots \ldots \ldots \ldots \ldots$

1.5 O Teorema de Radon-Nikodým . . . . . . . . . . . . . . . . . . . . . . 9

2 Sobre isomorfismos entre espaços $C_{0}(K, X)$ e a cardinalidade de $K \quad 15$

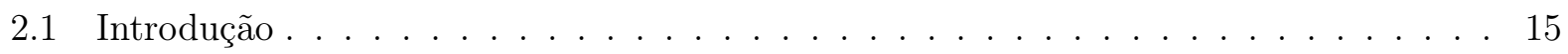

2.2 Sobre espaços $C_{0}(K, X), X$ de cotipo finito $\ldots \ldots \ldots \ldots \ldots$

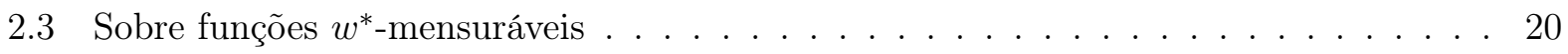

2.4 Sobre funções semicontínuas inferiormente . . . . . . . . . . . . . . . . . . 22

2.5 Demonstração do Teorema $2.8 \ldots \ldots \ldots \ldots \ldots \ldots \ldots \ldots$

3 Sobre isomorfismos entre espaços $C(K, X)$ com distorção menor que $3 \quad 27$

3.1 Introdução . . . . . . . . . . . . . . . . . . . . . . 27

3.2 Sobre isomorfismos de $C\left(K_{1}\right)$ em espaços $C\left(K_{2}, X\right) \ldots \ldots \ldots \ldots \ldots$

3.3 Demonstrações dos teoremas . . . . . . . . . . . . . . . . . . . . . . . 32

3.4 Considerações finais . . . . . . . . . . . . . . . . . . . . 35

4 Sobre distâncias de Banach-Mazur entre espaços $C_{0}(K, X)$ e $C_{0}(\Gamma, X) \quad 39$

4.1 Introdução . . . . . . . . . . . . . . . . . . . . . . 39

4.2 Resultados preliminares . . . . . . . . . . . . . . . . . . . . 41

4.3 Cotas inferiores para as distâncias entre $C_{0}(K, X)$ e $C_{0}(K, X) \ldots \ldots \ldots$. . . . 43

4.4 Cotas superiores as para distâncias entre $C\left(\left[1, \omega^{n} k\right], X\right)$ e $C_{0}(\mathbb{N}, X) \ldots \ldots$. . . . 47

5 Sobre distâncias de Banach-Mazur entre espaços $C(\omega)$ e $C(\alpha), \omega \leq \alpha<\omega^{\omega} \quad 53$

5.1 Introdução . . . . . . . . . . . . . . . . . . . . . . . 53

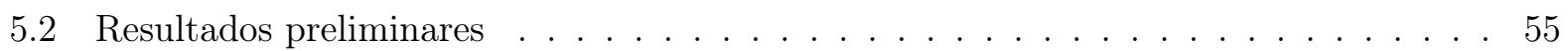

5.3 Cotas inferiores para as distâncias entre $C(K)$ e $C(L), L^{(2)}=\varnothing \ldots \ldots \ldots \ldots$ 
5.4 Cotas superiores para as distâncias entre $C(\omega)$ e $C\left(\omega^{n} k\right), 1 \leq n, k<\omega \ldots \ldots$. . . 59

Referências Bibliográficas 


\section{Lista de Símbolos}

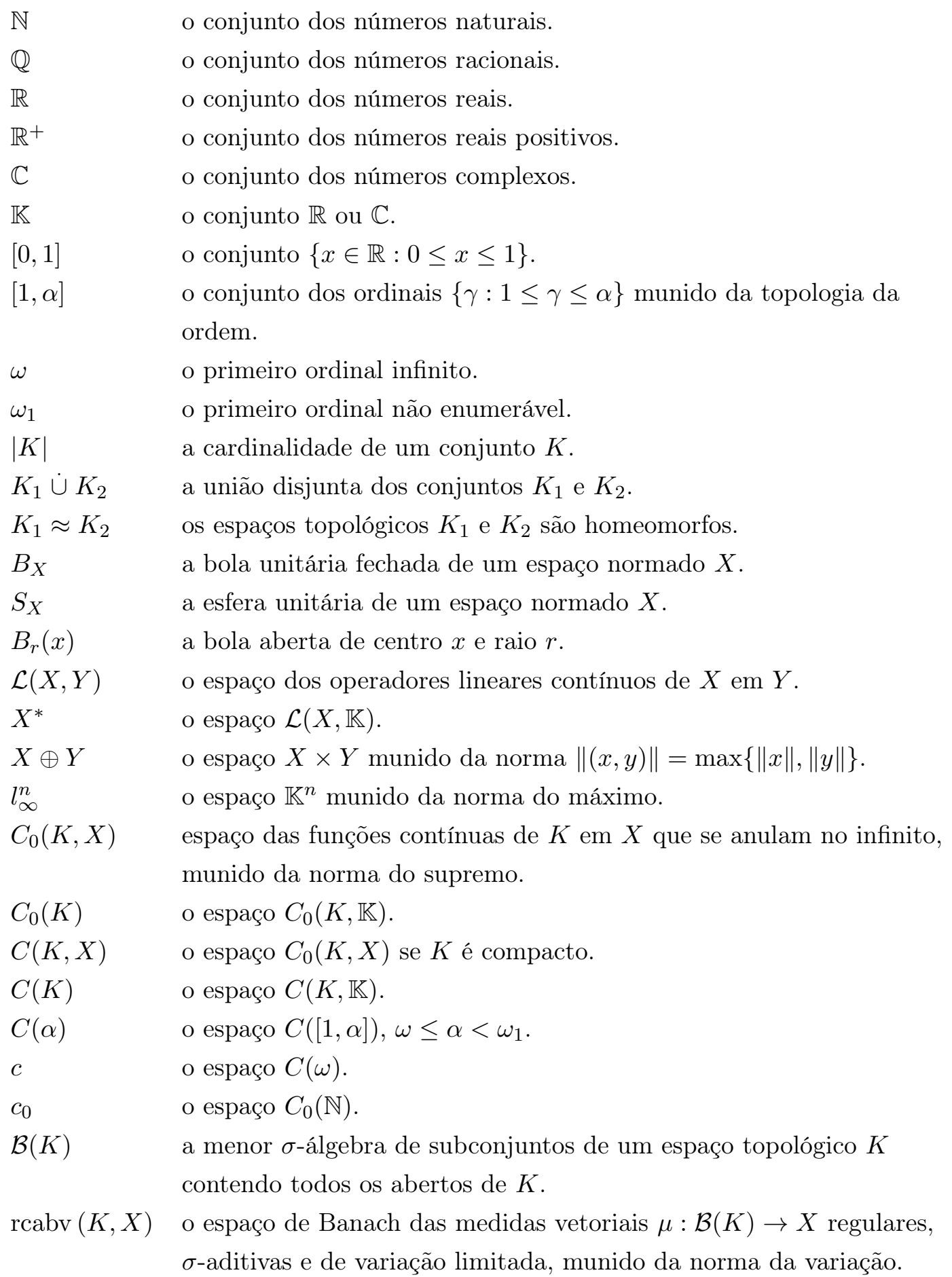


$\mathbb{1}_{A} \quad$ a função característica de um conjunto $A$.

$X \sim Y \quad$ os espaços de Banach $X$ e $Y$ são isomorfos .

$X \stackrel{\lambda}{\sim} Y$ existe um isomorfismo linear sobrejetor $T$ de $X$ em $Y$ tal que $\|T\|\left\|T^{-1}\right\|<\lambda$, para algum $1<\lambda<\infty$.

$X \hookrightarrow Y \quad$ o espaço de Banach $Y$ contém um subespaço isomorfo a $X$. 


\section{Introdução}

Neste trabalho estudamos alguns aspectos da teoria isomorfa dos espaços de $\operatorname{Banach} C_{0}(K, X)$. Um desses aspectos remonta ao clássico teorema de Banach-Stone:

Teorema (Banach-Stone). Sejam $K_{1}$ e $K_{2}$ espaços compactos de Hausdorff. Se $C\left(K_{1}\right)$ e $C\left(K_{2}\right)$ são isometricamente isomorfos, então $K_{1}$ e $K_{2}$ são homeomorfos.

Este resultado, obtido em 1932 por Banach [4] para compactos métricos e estendido em 1937 por Stone [50] para espaços compactos, foi generalizado em 1965 por Amir [3] para espaços compactos e, de forma independente, em 1967 por Cambern [13] para espaços localmente compactos. O resultado estabelece que se $K_{1}$ e $K_{2}$ são espaços localmente compactos de Haudorff e $T$ é um isomorfismo linear sobrejetor de $C_{0}\left(K_{1}\right)$ em $C_{0}\left(K_{2}\right)$, se $\|T\|\left\|T^{-1}\right\|<2$, então $K_{1}$ e $K_{2}$ são homeomorfos.

O teorema de Banach-Stone também foi generalizado para a classe dos espaços de Banach $C_{0}(K, X)$. Para espaços de Banach reais, a maior generalização até o presente momento, foi obtida em 1988 e é devida a Behrends e Cambern [6]. O resultado propõe que se $X$ é uniformemente não-quadrado, ver [33, Definição 1.1], existe $1<\lambda \leq 2$ tal que se $K_{1}$ e $K_{2}$ são espaços localmente compactos de Haudorff e $T$ é um isomorfismo linear sobrejetor de $C_{0}\left(K_{1}, X\right)$ em $C_{0}\left(K_{2}, X\right)$ com $\|T\|\left\|T^{-1}\right\|<\lambda$, então $K_{1}$ e $K_{2}$ são homeomorfos.

No que se refere às relações conjuntísticas preservadas entre espaços localmente compactos de Hausdorff $K_{1}$ e $K_{2}$ sob um isomorfismo sobrejetor $T$ de $C_{0}\left(K_{1}\right)$ em $C_{0}\left(K_{2}\right)$, um resultado não trivial obtido em 1978 por Cengiz [16], que de certa forma pode ser considerado uma versão fraca do teorema de Banach-Stone, estabelece que se os espaços $C_{0}\left(K_{1}\right)$ e $C_{0}\left(K_{2}\right)$ são isomorfos, então $K_{1}$ e $K_{2}$ têm a mesma cardinalidade.

Os resultados de Cengiz [16], Behrends e Cambern [6] sugerem o seguinte problema:

Problema. Sejam $X$ um espaço de Banach uniformemente não-quadrado, $K_{1}$ e $K_{2}$ espaços localmente compactos de Hausdorff. Suponha que $C_{0}\left(K_{1}, X\right)$ e $C_{0}\left(K_{2}, X\right)$ sejam isomorfos. Nessas condições o que pode ser dito sobre as cardinalidades de $K_{1}$ e $K_{2}$ ?

No Capítulo 2 respondemos completamente esta questão, mais ainda, obtemos um resultado envolvendo uma classe mais geral de espaços de Banach, os espaços de cotipo finito, ver [21, p. 218].

Ainda nesta linha de pesquisa devemos recordar um resultado de Gordon de 1970, ver [31]. Para uma certa classe de espaços compactos, o autor estabelece outra extensão para o Teorema de BanachStone. Mais precisamente, se $K_{1}$ e $K_{2}$ são compactos métricos enumeráveis e $T$ é um isomorfismo linear sobrejetor de $C\left(K_{1}\right)$ em $C\left(K_{2}\right)$ com $\|T\|\left\|T^{-1}\right\|<3$, então $K_{1}$ e $K_{2}$ são homeomorfos. 
No Capítulo 3, inspirado em Gordon [31], buscamos extensões de seu resultado para $C(K, X)$, onde $K$ é um compacto homeomorfo a um intervalo de ordinal e $X$ é um espaço de Banach. Nosso objetivo é resolver o seguinte problema:

Problema. Sejam $K_{1}$ e $K_{2}$ espaços compactos métricos enumeráveis e $X$ um espaço de Banach de cotipo finito. Suponha existir um isomorfismo sobrejetor $T$ de $C\left(K_{1}, X\right)$ em $C\left(K_{2}, X\right)$ com $\|T\|\left\|T^{-1}\right\|<3$. Nestas condições, o que pode ser dito a respeito de $K_{1}$ e $K_{2}$ ? Para algum desses espaços $X$ seria possível concluir que $K_{1}$ e $K_{2}$ são homeomorfos?

No Capítulo 4 abordamos outro aspecto da teoria isomorfa dos espaços de Banach $C_{0}(K, X)$. Desta vez, dado um isomorfismo sobrejetor $T$ de $C_{0}\left(K_{1}\right)$ em $C_{0}\left(K_{2}\right)$, sabendo que os espaços $K_{1}$ e $K_{2}$ não são homeomorfos procuramos quantificar a distorção $\|T\|\left\|T^{-1}\right\|$. Estudamos inicialmente o caso $K_{2}=\mathbb{N}$. Nesse sentido, voltamos nossa atenção para um tabalho de Cambern de 1968, ver [14], no qual foi estabelecido que a distância de Banach-Mazur entre os espaços $c$ e $c_{0}$ é exatamente 3. Recorde que para espaços de Banach isomorfos $X$ e $Y$ a distância de Banach-Mazur é definida por

$$
\mathrm{d}(X, Y)=\inf _{T}\left\{\|T\|\left\|T^{-1}\right\|\right\}
$$

onde $T$ percorre todos os isomorfismos sobrejetores de $X$ em $Y$. O resultado de Cambern [14] sugere o seguinte problema:

Problema. Quais são os valores de $\mathrm{d}\left(C\left(\left[1, \omega^{n} k\right]\right), c_{0}\right), 1 \leq n, k<\omega$ ?

Mais geralmente, se $\Gamma$ é um espaço topológico discreto e $K$ é um espaço localmente compacto de Hausdorff não discreto, a intuição sugere que um isomorfimo sobrejetor $T$ de $C_{0}(K)$ em $C_{0}(\Gamma)$ deve "destruir" os pontos de acumulação de $K$ e, por isso, é razoável pensar que $\|T\|\left\|T^{-1}\right\|$ cresce conforme aumenta a altura de $K$. De fato, no Capítulo 4 demonstramos que para um espaço de Banach $X$ de cotipo finito, se $T$ é um isomorfismo sobrejetor de $C_{0}(K, X)$ em $C_{0}(\Gamma, X)$ e se o $n$ ésimo derivado de $K$ for não vazio, então $\|T\|\left\|T^{-1}\right\| \geq 2 n+1$. Também apresentamos uma solução completa para o problema anterior.

No Capítulo 5, seguindo a mesma linha de trabalho do Capítulo 4, estudamos os espaços de Banach $C(\alpha), \omega \leq \alpha<\omega_{1}$. A classificação isomorfa destes espaços foi obtida em 1960 por Bessaga e Pełczyński [7]. Eles estabeleceram que se $\omega \leq \alpha \leq \beta<\omega_{1}$, então $C(\alpha)$ é isomorfo a $C(\beta)$ se e somente se existe $1 \leq n<\omega$ tal que $\alpha^{n} \leq \beta<\alpha^{n+1}$. Na ocasião, também foram obtidas estimativas para as distâncias de Banach-Mazur entre estes espaços: $n \leq \mathrm{d}(C(\alpha), C(\beta)) \leq 4^{n+3}$.

Bessaga e Pełczyński [7, p. 59] consideram o problema de se obter funções $G, H: \mathbb{N} \rightarrow \mathbb{R}^{+}$ satisfazendo

$$
\sup (H(n) / G(n))<\infty \quad \text { e } \quad G(n) \leq \mathrm{d}(C(\alpha), C(\beta)) \leq H(n), n \in \mathbb{N} .
$$

Inspirado em resultados de Cambern [14] e utilizando técnicas desenvolvidas no Capítulo 4, apresentamos estimativas como acima para o caso $\alpha=\omega$. Estas estimativas nos levam a conjecturar os valores exatos das distâncias de Banach-Mazur entre os espaços $C(\omega)$ e $C\left(\omega^{n} k\right), 1 \leq n, k<\omega$. 


\section{Capítulo 1}

\section{Medidas vetoriais e integração vetorial}

\subsection{Introdução}

Nosso objetivo neste capítulo é reunir algumas ferramentas técnicas necessárias para abordar o estudo de funcionais lineares definidos sobre espaços de funções a valores vetoriais. Vamos apresentar algumas definições e propriedades básicas em teoria de medidas vetoriais e espaços de funções contínuas que serão utilizadas ao longo deste trabalho. Para um estudo mais detalhado sobre estes tópicos recomendamos [20], [22] e [23].

\subsection{Medidas Vetoriais}

Em princípio, utilizaremos a notação e a terminologia para teoria de medida e teoria de espaços de Banach que podem ser encontradas em [20] e [35]. Iniciamos com a seguinte definição:

Definição 1.1. Sejam $K$ um conjunto não vazio, $\mathcal{A}$ uma álgebra de subconjuntos de $K$ e $X$ um espaço normado. Uma função $\mu: \mathcal{A} \rightarrow X$ é denominada medida vetorial finitamente aditiva se verificar para quaisquer $A, B \in \mathcal{A}$, com $A \cap B=\emptyset$,

$$
\mu(A \cup B)=\mu(A)+\mu(B) .
$$

Uma função $\mu: \mathcal{A} \rightarrow X$ é denominada medida vetorial $\sigma$-aditiva se para toda sequência $\left(A_{i}\right)_{i \in \mathbb{N}}$ de elementos mutuamente disjuntos de $\mathcal{A}$ com união em $\mathcal{A}$, verificar

$$
\mu\left(\bigcup_{i=1}^{\infty} A_{i}\right)=\sum_{i=1}^{\infty} \mu\left(A_{i}\right) .
$$

Observação 1.2. Estaremos focados principalmente em medidas vetoriais $\sigma$-aditivas definidas em $\sigma$-álgebras. Contudo, o conceito de medida finitamente aditiva é fundamental na investigação de operadores lineares definidos sobre espaços de funções a valores vetoriais. Por simplicidade e sem possibilidade de confusão, por medida vetorial estaremos nos referindo tanto a uma medida finitamente aditiva quanto a uma medida $\sigma$-aditiva nas condições da Definição 1.1.

Um par ordenado $(K, \Sigma)$, onde $K$ é um conjunto e $\Sigma$ é uma $\sigma$-álgebra sobre $K$, será chamado espaço mensurável.

Muitos dos resultados obtidos neste trabalho dependem do conceito de variação que recordaremos em seguida.

Definição 1.3. Sejam $(K, \Sigma)$ um espaço mensurável, $X$ um espaço de Banach e $\mu: \Sigma \rightarrow X$ uma 
medida vetorial. A variação de $\mu$ é a aplicação $|\mu|$ definida sobre $\Sigma$ por

$$
|\mu|(A)=\sup \sum_{k=1}^{n}\left\|\mu\left(A_{k}\right)\right\|, A \in \Sigma,
$$

onde o supremo é tomado sob todas as partições finitas $\left\{A_{1}, \ldots, A_{n}\right\}$ de $A$ em $\Sigma$. Sempre que uma medida vetorial $\mu$ satisfizer $|\mu|(K)<\infty$, diremos que $\mu$ tem variação limitada.

Proposição 1.4. Sejam $(K, \Sigma)$ um espaço mensurável e $X$ um espaço de Banach. Se $\mu: \Sigma \rightarrow X$ é uma medida vetorial de varição limitada e $\sigma$-aditiva (finitamente aditiva), sua variação $|\mu|: \Sigma \rightarrow \mathbb{R}$ é uma medida positiva e $\sigma$-aditiva (finitamente aditiva). Mais ainda, $|\mu|$ é a menor de todas as medidas positivas que cumprem $\mu(A) \leq|\mu|(A)$ para todo $A \in \Sigma$.

Demonstração. Claramente $|\mu|(A) \geq 0$ para todo $A \in \Sigma$ e $|\mu|(A) \leq|\mu|(B)$ sempre que $A, B \in \Sigma$ e $A \subseteq B$.

Vamos verificar o caso em que $\mu$ é $\sigma$-aditiva. Para $\mu$ finitamente aditiva, a demonstração pode ser obtida com um argumento análogo.

Seja $\left(A_{i}\right)_{i \in \mathbb{N}}$ uma sequência de elementos mutuamente disjuntos de $\Sigma$. Dado $\epsilon>0$ arbitrário, para cada $i \in \mathbb{N}$, seja $\left\{B_{1}^{i}, \ldots, B_{n_{i}}^{i}\right\}$ uma partição de $A_{i}$ em $\Sigma$ satisfazendo

$$
\sum_{j=1}^{n_{i}}\left\|\mu\left(B_{j}^{i}\right)\right\| \geq|\mu|\left(A_{i}\right)-\frac{\epsilon}{2^{i}}
$$

Então, para qualquer subconjunto finito $J \subset \mathbb{N}$ podemos escrever

$$
|\mu|\left(\bigcup_{i=1}^{\infty} A_{i}\right) \geq|\mu|\left(\bigcup_{i \in J} A_{i}\right) \geq \sum_{i \in J}\left(\sum_{j=1}^{n_{i}}\left\|\mu\left(B_{j}^{i}\right)\right\|\right) \geq \sum_{i \in J}|\mu|\left(A_{i}\right)-\epsilon .
$$

Isso implica

$$
|\mu|\left(\bigcup_{i=1}^{\infty} A_{i}\right) \geq \sum_{i=1}^{\infty}|\mu|\left(A_{i}\right)
$$

Por outro lado, dado $\epsilon>0$ arbitrário, seja $\left\{B_{1}, \ldots, B_{n}\right\}$ uma partição de $\bigcup_{i=1}^{\infty} A_{i}$ verificando

$$
\sum_{j=1}^{n}\left\|\mu\left(B_{j}\right)\right\| \geq|\mu|\left(\bigcup_{i=1}^{\infty} A_{i}\right)-\epsilon
$$

Claramente, para cada $i \in \mathbb{N}$, a coleção $\left\{A_{i} \cap B_{1}, \ldots, A_{i} \cap B_{n}\right\}$ forma uma partição de $A_{i}$ em $\Sigma$. Podemos escrever

$$
\left\|\mu\left(B_{j}\right)\right\|=\left\|\mu\left(\bigcup_{i=1}^{\infty}\left(A_{i} \cap B_{j}\right)\right)\right\|=\left\|\sum_{i=1}^{\infty} \mu\left(A_{i} \cap B_{j}\right)\right\| \leq \sum_{i=1}^{\infty}\left\|\mu\left(A_{i} \cap B_{j}\right)\right\|, 1 \leq j \leq n .
$$

Consequentemente

$$
|\mu|\left(\bigcup_{i=1}^{\infty} A_{i}\right)-\epsilon \leq \sum_{j=1}^{n}\left\|\mu\left(B_{j}\right)\right\| \leq \sum_{i=1}^{\infty}\left(\sum_{j=1}^{n}\left\|\mu\left(A_{i} \cap B_{j}\right)\right\|\right) \leq \sum_{i=1}^{\infty}|\mu|\left(A_{i}\right) .
$$


Como $\epsilon>0$ é arbitrário, deduzimos que

$$
|\mu|\left(\bigcup_{i=1}^{\infty} A_{i}\right) \leq \sum_{i=1}^{\infty}|\mu|\left(A_{i}\right) .
$$

Para a segunda parte, seja $\lambda$ outra medida positiva satisfazendo $\|\mu(A)\| \leq \lambda(A)$ para todo $A \in \Sigma$. Então, dada uma partição $\left\{A_{1}, \ldots, A_{n}\right\}$ arbitrária de $A$ em $\Sigma$, temos

$$
\sum_{i=1}^{n}\left\|\mu\left(A_{i}\right)\right\| \leq \sum_{i=1}^{n} \lambda\left(A_{i}\right)=\lambda(A),
$$

$\log$,

$$
|\mu|(A) \leq \lambda(A)
$$

\subsection{Integração vetorial}

Apresentaremos brevemente nesta seção um conceito simples de integração vetorial que terá um papel importante em nosso trabalho. Trata-se de um conceito de integração sobre medidas vetoriais a valores em duais de espaços normados. Para um estudo mais detalhado sobre este tópico recomendamos [22].

Nesta seção e ao longo deste trabalho, para um conjunto arbitrário $A$, denotaremos por $\mathbb{1}_{A}$ a função característica de $A$.

Definição 1.5. Sejam $(K, \Sigma)$ um espaço mensurável e $X$ um espaço de Banach. Uma função $f: K \rightarrow X$ será chamada de simples se existirem $v_{1}, \ldots, v_{n} \in X$ e $A_{1}, \ldots, A_{n} \in \Sigma$ tais que

$$
f=\sum_{i=1}^{n} v_{i} \cdot \mathbb{1}_{A_{i}} .
$$

Observação. É possível verificar que toda função simples $f: K \rightarrow X$ pode ser escrita como

$$
f=\sum_{i=1}^{n} v_{i} \cdot \mathbb{1}_{A_{i}},
$$

onde $v_{1}, \ldots, v_{n} \in X$ e $A_{1}, \ldots, A_{n}$ formam uma partição de $K$ em $\Sigma$.

Para um espaço mensurável $(K, \Sigma)$ e um espaço de Banach $X$, o conjunto de todas as funções simples de $K$ em $X$, munido das operações usuais de adição de funções e multiplicação de função por escalar, é um espaço vetorial. A aplicação $f \mapsto\|f\|=\sup _{x \in K}\|f(x)\|$ define uma norma sobre este espaço. Este espaço normado será denotado por $\mathcal{S}(\Sigma, X)$.

Definição 1.6. Sejam $(K, \Sigma)$ um espaço mensurável e $X$ um espaço de Banach. Dizemos que uma função $f: K \rightarrow X$ é mensurável se existe uma sequência $\left(f_{n}\right)_{n \in \mathbb{N}}$ em $\mathcal{S}(\Sigma, X)$ tal que $f_{n} \rightarrow f$ pontualmente.

Teorema 1.7. Sejam $(K, \Sigma)$ um espaço mensurável e $X$ um espaço de Banach. Se $\left(f_{n}\right)_{n \in \mathbb{N}}$ é uma sequência de funções de $K$ em $X$, mensuráveis e convergindo pontualmente a $f$, então o limite $f$ é mensurável.

Demonstração. Ver N. Dinculeanu [22, Teorema 10, p. 6]. 
Medidas vetoriais a valores em duais topológicos de espaços normados desempenham papel fundamental no estudo de funcionais lineares contínuos sobre espaços de funções a valores vetoriais. A este tipo de medida dedicaremos nosso subsequente estudo neste capítulo.

Para um espaço de Banach $X$ denotamos $X^{*}$ seu dual topológico, i.e., o espaço de todos os funcionais lineares contínuos em $X$. A dualidade entre $X^{*}$ e $X$ será denotada por $\left\langle v^{*}, v\right\rangle,\left\langle v, v^{*}\right\rangle$ ou $v^{*}(v)$.

Proposição 1.8. Sejam $(K, \Sigma)$ um espaço mensurável e $X$ um espaço de Banach. Se $\mu: \Sigma \rightarrow X^{*}$ é uma medida vetorial de variação limitada, então

$$
|\mu|(A)=\sup \left|\sum_{i=1}^{n}\left\langle\mu\left(A_{i}\right), v_{i}\right\rangle\right|, A \in \Sigma,
$$

onde o supremo é tomado sob todas as partições finitas $\left\{A_{1}, \ldots, A_{n}\right\}$ de $A$ em $\Sigma$ e $v_{1}, \ldots, v_{n} \in B_{X}$.

Demonstração. Fixe $A \in \Sigma$ arbitrário. Da definição de variação temos

$$
|\mu|(A) \geq \sup \left|\sum_{i=1}^{n}\left\langle\mu\left(A_{i}\right), v_{i}\right\rangle\right|
$$

Resta apenas demonstrar a desigualdade contrária. Fixe $\epsilon>0$ e uma partição qualquer $\left\{B_{1}, \ldots, B_{m}\right\}$ de $A$. Para cada $1 \leq j \leq m$ existe $u_{j} \in B_{X}$ satisfazendo

$$
\left\|\mu\left(B_{j}\right)\right\|-\frac{\epsilon}{m} \leq\left\langle\mu\left(B_{j}\right), u_{j}\right\rangle
$$

Então

$$
\sum_{j=1}^{m}\left\|\mu\left(B_{j}\right)\right\|-\epsilon \leq \sum_{j=1}^{m}\left\langle\mu\left(B_{j}\right), u_{j}\right\rangle \leq \sup \left|\sum_{i=1}^{n}\left\langle\mu\left(A_{i}\right), v_{i}\right\rangle\right|
$$

e deduzimos que

$$
|\mu|(A) \leq \sup \left|\sum_{i=1}^{n}\left\langle\mu\left(A_{i}\right), v_{i}\right\rangle\right|+\epsilon
$$

Por $\epsilon>0$ ser arbitrário concluímos que

$$
|\mu|(A) \leq \sup \left|\sum_{i=1}^{n}\left\langle\mu\left(A_{i}\right), v_{i}\right\rangle\right|
$$

Sejam $(K, \Sigma)$ um espaço mensurável e $\mu: \Sigma \rightarrow X^{*}$ uma medida vetorial de variação limitada. Para cada $f=\sum_{i=1}^{n} v_{i} \cdot \mathbb{1}_{A_{i}} \in \mathcal{S}(\Sigma, X)$ defina

$$
S_{\mu} f=\sum_{i=1}^{n}\left\langle\mu\left(A_{i}\right), v_{i}\right\rangle
$$

Em virtude de $\mu$ ser finitamente aditiva, o valor de $S_{\mu} f$ independe da particular representação de $f$ como uma função simples. Não é difícil verificar que a fórmula (1.1) define um funcional linear contínuo em $\mathcal{S}(\Sigma, X)$ e que, de acordo com a Proposição 1.8, verifica

$$
\left\|S_{\mu}\right\|=|\mu|(K)
$$


Denotando por $\mathcal{M}(\Sigma, X)$ o completamento de $\mathcal{S}(\Sigma, X)$, espaço cujos os elementos serão denominados funções totalmente mensuráveis, o funcional $S_{\mu}$ admite uma única extensão de mesma norma a $\mathcal{M}(\Sigma, X)$ que será denotada por

$$
\int f d \mu, f \in \mathcal{M}(\Sigma, X)
$$

Da mesma forma, dado $A \in \Sigma$, a aplicação $\mu_{A}(B)=\mu(B \cap A)$, define uma medida sobre $\Sigma$. O funcional linear associado a $\mu_{A}$ tem norma $|\mu|(A)$ e pode ser denotado

$$
\int_{A} f d \mu=\int f d \mu_{A}, f \in \mathcal{M}(\Sigma, X) .
$$

Se $A, B \in \Sigma$ são disjuntos, então $\mu_{A \cup B}=\mu_{A}+\mu_{B}$. Com a notação acima podemos escrever

$$
\int_{A \cup B} f d \mu=\int f d \mu_{A \cup B}=\int f d \mu_{A}+\int f d \mu_{B}=\int_{A} f d \mu+\int_{B} f d \mu, f \in \mathcal{M}(\Sigma, X) .
$$

No caso escalar, no qual $X=\mathbb{R}$ ou $X=\mathbb{C}$, para funções totalmente mensuráveis a integração definida acima coincide com a integração abstrata usual desenvolvida em teoria da medida. A proposição seguinte combina esses dois conceitos.

Proposição 1.9. Sejam $(K, \Sigma)$ um espaço mensurável e $\mu: \Sigma \rightarrow X^{*}$ uma medida vetorial de variação limitada. Para cada $f \in \mathcal{M}(\Sigma, X)$ temos

$$
\left|\int f d \mu\right| \leq \int\|f(x)\| d|\mu|(x)
$$

Demonstração. Seja $f \in \mathcal{M}(\Sigma, X)$. Se $f$ é simples, suponha $f=\sum_{i=1}^{n} v_{i} \cdot \mathbb{1}_{A_{i}}$ onde $A_{1}, \ldots, A_{n} \in \Sigma$ são mutuamente disjuntos. Então

$$
\begin{aligned}
\left|\int f d \mu\right| & =\left|\sum_{i=1}^{n}\left\langle\mu\left(A_{i}\right), v_{i}\right\rangle\right| \leq \sum_{i=1}^{n}\left|\left\langle\mu\left(A_{i}\right), v_{i}\right\rangle\right| \leq \sum_{i=1}^{n}\left\|\mu\left(A_{i}\right)\right\|\left\|v_{i}\right\| \\
& \leq \sum_{i=1}^{n}\left\|v_{i}\right\||\mu|\left(A_{i}\right)=\int\|f(x)\| d|\mu|(x) .
\end{aligned}
$$

Para o caso geral, seja $\left(f_{n}\right)_{n \in \mathbb{N}}$ uma sequência em $\mathcal{S}(\Sigma, X)$ convergindo uniformemente a $f$. Da continuidade do funcional $g \mapsto \int g d \mu$ temos

$$
\lim _{n \rightarrow+\infty}\left|\int f_{n} d \mu\right|=\left|\int f d \mu\right|
$$

e como claramente $\left(\left\|f_{n}\right\|\right)_{n \in \mathbb{N}}$ converge uniformemente a $\|f\|$ podemos escrever

$$
\lim _{n \rightarrow+\infty} \int\left\|f_{n}(x)\right\| d|\mu|(x)=\int\|f(x)\| d|\mu|(x) .
$$

Já verificamos a proposição para funções simples. Assim,

$$
\left|\int f_{n} d \mu\right| \leq \int\left\|f_{n}(x)\right\| d|\mu|(x), n \in \mathbb{N} .
$$

Combinando as relações (1.2), (1.3) e (1.4) temos 


$$
\left|\int f d \mu\right|=\lim _{n \rightarrow+\infty}\left|\int f_{n} d \mu\right| \leq \lim _{n \rightarrow+\infty} \int\left\|f_{n}(x)\right\| d|\mu|(x)=\int\|f(x)\| d|\mu|(x) .
$$

\subsection{Representação de funcionais em $C_{0}(K, X)$}

Nossa meta nesta seção é apresentar um breve estudo sobre funcionais lineares definidos em determinados espaços de funções contínuas. Como veremos na sequência, este estudo está intimamente ligado com o conceito de integração apresentado na Seção 1.3.

Definição 1.10. Sejam $K$ um espaço topológico localmente compacto de Hausdorff e $X$ um espaço de Banach. Dizemos que uma função $f: K \rightarrow X$ se anula no infinito se para cada $\epsilon>0$ existe um compacto $J \subset K$ tal que $\|f(x)\|<\epsilon$ para todo $x \in K \backslash J$.

Para um espaço localmente compacto de Hausdorff $K$ e um espaço de Banach $X$, o conjunto de todas as funções contínuas $f: K \rightarrow X$ que se anulam no infinito, munido das operações usuais de adição de funções e multiplicação de função por escalar, formam um espaço vetorial, mais ainda, a aplicação $f \mapsto\|f\|=\sup _{x \in K}\|f(x)\|$ define uma norma completa sobre este espaço. Este espaço de Banach será denotado por $C_{0}(K, X)$. Se $X$ for $\mathbb{R}$ ou $\mathbb{C}$, este espaço será denotado por $C_{0}(K)$. Quando $K$ for compacto estes espaços serão denotados por $C(K, X)$ e $C(K)$ respectivamente.

Observação 1.11. Sejam $K$ um espaço topológico localmente compacto de Hausdorff não compacto, $\bar{K}=K \dot{\cup}\{\infty\}$ o compactificado de Aleksandrov de $K$ e $X$ um espaço de Banach. O espaço $C_{0}(K, X)$ pode ser identificado de maneira natural com o subespaço de $C(\bar{K}, X)$ das funções contínuas $f: \bar{K} \rightarrow X$ satisfazendo $f(\infty)=0$.

Teorema 1.12 (Lema de Urysohn). Sejam K um espaço localmente compacto de Hausdorff, $U$ um aberto em $K, F \subset U$ e $F$ é compacto. Então existe $f \in C_{0}(K)$ tal que $0 \leq f(x) \leq 1$ para todo $x \in K, f(x)=1$ se $x \in F$ e $f(x)=0$ se $x \in K \backslash U$.

Demonstração. Se $K$ for compacto, por $K$ ser de Hausdorff, então $K$ é normal. Podemos aplicar o Lema de Urysohn para espaços topológicos normais [25, Teorema 1.5.11] e concluir que existe $f \in C(K)$ tal que $0 \leq f(x) \leq 1$ para todo $x \in K, f(x)=1$ se $x \in F$ e $f(x)=0$ se $x \in K \backslash U$.

Se $K$ for não compacto, como na Observação 1.11, identificamos o espaço $C_{0}(K)$ com o subespaço de $C(\bar{K})$ das funções contínuas $f: \bar{K} \rightarrow \mathbb{K}$ satisfazendo $f(\infty)=0$. Por $K$ ser localmente compacto de Hausdorff, seu compactificado de Aleksandrov $\bar{K}$ é normal. Por $F \subset K$ ser compacto, o conjunto $F$ é fechado em $\bar{K}$. Podemos aplicar novamente o [25, Teorema 1.5.11] e obter uma função $f \in C(\bar{K})$ tal que $0 \leq f(x) \leq 1$ para todo $x \in \bar{K}, f(x)=1$ se $x \in F$ e $f(x)=0$ se $x \in \bar{K} \backslash U$. Por $f(\infty)=0$, temos que $f \in C_{0}(K)$.

Para um espaço topológico $K$, a menor $\sigma$-álgebra contendo todos os abertos de $K$ é chamada $\sigma$-álgebra de Borel e será denotada por $\mathcal{B}(K)$. Os elementos de $\mathcal{B}(K)$ serão chamados de borelianos de $K$. Uma medida vetorial ou escalar $\mu$ definida sobre $\mathcal{B}(K)$ será chamada de medida de Borel.

Para medidas de Borel, um conceito fundamental em nosso trabalho é o de regularidade que recordaremos em seguida.

Definição 1.13. Seja $K$ um espaço topológico. Uma medida positiva $\mu: \mathcal{B}(K) \rightarrow \mathbb{R}$ será denominada regular se para todo boreliano $B$, satisfizer

$$
\mu(B)=\inf \{\mu(U): B \subset U, U \text { é aberto }\}=\sup \{\mu(L): L \subset B, L \text { é compacto }\} .
$$


Se $\mu$ for uma medida vetorial de Borel, diremos que $\mu$ é regular quando $|\mu|$ for regular no sentido acima.

Sejam $K$ um espaço localmente compacto de Hausdorff e $X$ um espaço de Banach. O conjunto de todas medidas $\mu: \mathcal{B}(K) \rightarrow X$ regulares, $\sigma$-aditivas e de variação limitada, munido das operações usuais de adição de medidas e multiplicação de medida por escalar, é um espaço vetorial. A aplicação $\mu \mapsto\|\mu\|=|\mu|(K)$ define uma norma completa sobre este espaço que será denotado por $\operatorname{rcabv}(K, X)$. Se $X$ for $\mathbb{R}$ ou $\mathbb{C}$ este espaço será denotado simplesmente por $\operatorname{rcabv}(K)$.

Observação 1.14. Por simplicidade, para um espaço topológico $K$ localmente compacto de Hausdorff estaremos assumindo de maneira implícita a $\sigma$-álgebra $\mathcal{B}(K)$. Também por simplicidade, para o espaço mensurável $(K, \mathcal{B}(K))$ e um espaço de Banach $X$, o espaço das funções simples mensuráveis munido da norma do supremo, introduzido na Seção 1.3, será denotado por $\mathcal{S}(K, X)$ e seu completamento por $\mathcal{M}(K, X)$.

Proposição 1.15. Sejam $K$ um espaço localmente compacto de Hausdorff e $X$ um espaço de Banach. Para toda função $f \in C_{0}(K, X)$ existe uma sequência $\left(f_{n}\right)_{n \in \mathbb{N}}$ em $\mathcal{S}(K, X)$ convergindo uniformemente a $f$ e tal que $\left\|f_{n}\right\| \leq\|f\|$ para todo $n \in \mathbb{N}$.

Demonstração. Seja $f \in C_{0}(K, X)$ e sem perda de generalidade vamos supor que $\|f\|=1$. Fixado $n \in \mathbb{N}$, considere o conjunto $L_{n}=\left\{x \in K:\|f(x)\| \geq \frac{1}{n}\right\}$ que é não vazio e compacto, pois a função $f$ é contínua e se anula no infinito. Para cada $x \in L_{n}$ defina

$$
U_{x}=f^{-1}\left(B_{\frac{1}{n}}(f(x))\right)
$$

Note-se que $\left\{U_{x}: x \in K\right\}$ é um recobrimento aberto de $L_{n}$. Por compacidade, existem pontos distintos $x_{1}, \ldots, x_{m} \in L_{n}$ tais que

$$
L_{n} \subset U_{x_{1}} \cup \ldots \cup U_{x_{m}} .
$$

Em seguida, para cada $1 \leq i \leq m$ defina

$$
A_{i}=U_{x_{i}} \backslash \bigcup_{j<i} U_{x_{j}} \quad \text { e } \quad f_{n}=\sum_{i=1}^{m} f\left(x_{i}\right) \cdot \mathbb{1}_{A_{i}} .
$$

Para cada $x \in K$, se $x \in K \backslash\left(U_{x_{1}} \cup \ldots \cup U_{x_{m}}\right)$, então $x \notin L_{n}$ e $f_{n}(x)=0$, logo

$$
\left\|f(x)-f_{n}(x)\right\|=\|f(x)\|<\frac{1}{n} .
$$

Por outro lado, se $x \in U_{x_{1}} \cup \ldots \cup U_{x_{m}}$, então existe um único $1 \leq i \leq m$ tal que $x \in A_{i}$. Temos

$$
\left\|f(x)-f_{n}(x)\right\|=\left\|f(x)-f\left(x_{i}\right)\right\|<\frac{1}{n} .
$$

Portanto $\left\|f-f_{n}\right\| \leq \frac{1}{n}$ e dessa forma, podemos construir uma sequência $\left(f_{n}\right)_{n \in \mathbb{N}}$ de funções simples convergindo uniformemente a $f$. Mais ainda, segue imediatamente da construção de cada $f_{n}$ que $\left\|f_{n}\right\| \leq\|f\|$.

Teorema 1.16. Sejam $K$ um espaço localmente compacto de Hausdorff e $\mu \in \operatorname{rcabv}\left(K, X^{*}\right)$. Para cada aberto $U \subseteq K$ seja $\mathcal{F}_{U}=\left\{f \in C_{0}(K, X):\|f\| \leq 1\right.$ e $f(x)=0$ se $\left.x \in K \backslash U\right\}$. Então

$$
|\mu|(U)=\sup _{\mathcal{F}_{U}}\left|\int f d \mu\right| .
$$


Demonstração. Seja $U \subseteq K$ um aberto não vazio. Dado $\epsilon>0$, de acordo com a Proposição 1.8, existe uma função simples $g=\sum_{i=1}^{n} v_{i} \cdot \mathbb{1}_{A_{i}}$, onde $v_{1}, \ldots, v_{n} \in B_{X}$ e $A_{1}, \ldots, A_{n}$ formam uma partição de $U$ em $\mathcal{B}(K)$, satisfazendo

$$
|\mu|(U)<\frac{\epsilon}{2}+\left|\sum_{i=1}^{n}\left\langle\mu\left(A_{i}\right), v_{i}\right\rangle\right|=\frac{\epsilon}{2}+\left|\int g d \mu\right| .
$$

Devido à regularidade de $|\mu|$, existem abertos $U_{1}, U_{2}, \ldots, U_{n}$ e compactos $J_{1}, J_{2}, \ldots, J_{n}$ satisfazendo

$$
J_{i} \subset A_{i} \subset U_{i} \subset U \text { e }|\mu|\left(U_{i} \backslash J_{i}\right)<\frac{\epsilon}{2 n\left(\left\|v_{i}\right\|+1\right)}, \quad 1 \leq i \leq n .
$$

Aplicando o Lema de Urysohn 1.12, podemos obter funções $h_{i} \in C_{0}(K), 1 \leq i \leq n$, tais que $0 \leq h_{i}(x) \leq 1$ para todo $x \in K, h_{i}(x)=1$ se $x \in J_{i}$ e $h_{i}(x)=0$ se $x \in K \backslash U_{i}$. Como os compactos $J_{1}, \ldots, J_{n}$ são mutuamente disjuntos, as funções $h_{1}, \ldots, h_{n}$ podem ser escolhidas com suportes mutuamente disjuntos. Defina $h: K \rightarrow X$ por

$$
h(x)=\sum_{i=1}^{n} v_{i} \cdot h_{i}(x) .
$$

Claramente $h \in \mathcal{F}_{U}$. Utilizando a Proposição 1.9 podemos escrever

$$
\begin{aligned}
\left|\int g d \mu-\int h d \mu\right| & =\left|\int(g-h) d \mu\right| \leq \int\|g-h\| d|\mu| \leq \sum_{i=1}^{n}\left(\left\|v_{i}\right\| \int\left|\mathbb{1}_{A_{i}}-h_{i}\right| d|\mu|\right) \\
& \leq \sum_{i=1}^{n}\left(\left\|v_{i}\right\| \int \mathbb{1}_{\left(U_{i} \backslash K_{i}\right)} d|\mu|\right) \leq \sum_{i=1}^{n}\left(\left\|v_{i}\right\| \cdot|\mu|\left(U_{i} \backslash K_{i}\right)\right) \\
& \leq \sum_{i=1}^{n} \frac{\left\|v_{i}\right\| \epsilon}{2 n\left(\left\|v_{i}\right\|+1\right)}<\frac{\epsilon}{2} .
\end{aligned}
$$

Então, retomando a relação (1.5), deduzimos

$$
|\mu|(U)<\frac{\epsilon}{2}+\left|\int g d \mu\right|<\epsilon+\left|\int h d \mu\right| \leq \epsilon+\sup _{\mathcal{F}_{U}}\left|\int f d \mu\right| .
$$

Por $\epsilon$ ser arbitrário, resulta

$$
|\mu|(U) \leq \sup _{\mathcal{F}_{U}}\left|\int f d \mu\right| .
$$

Por outro lado, fixe $f \in \mathcal{F}_{U}$ arbitrária. Se $f$ não for a função nula, podemos supor sem perda de generalidade que $\|f\|=1$. Neste caso, seja $\left(f_{n}\right)_{n \in \mathbb{N}}$ uma sequência em $\mathcal{S}(K, X)$, construída como na demonstração da Proposição 1.15, que converge uniformemente a $f$ e que satisfaz $\left\|f_{n}\right\| \leq 1$ para todo $n \in \mathbb{N}$.

Dado $n \in \mathbb{N}$, por $f_{n}(x)=0$ se $x \in K \backslash U$, podemos escrever $f_{n}=\sum_{i=1}^{r_{n}} v_{i} \cdot \mathbb{1}_{A_{i}}$, onde $v_{1}, \ldots, v_{r_{n}} \in$ $B_{X}$ e $A_{1}, \ldots, A_{r_{n}}$ formam uma partição de $U$ em $\mathcal{B}(K)$. Assim,

$$
\left|\int f_{n} d \mu\right|=\left|\sum_{i=1}^{r_{n}}\left\langle\mu\left(A_{i}\right), v_{i}\right\rangle\right| \leq|\mu|(U) .
$$


Passando-se ao limite na expressão acima para $n$ tendendo ao infinito obtemos

$$
\left|\int f d \mu\right| \leq|\mu|(U)
$$

Por $f \in \mathcal{F}_{U}$ ser arbitrária, temos

$$
\sup _{\mathcal{F}_{U}}\left|\int f d \mu\right| \leq|\mu|(U)
$$

Para um espaço localmente compacto de Hausdorff $K$ e um espaço de Banach $X$, de acordo com a Proposição 1.15, temos $C_{0}(K, X) \subset \mathcal{M}(K, X)$. Assim, dado um funcional linear contínuo $\varphi$ sobre $C_{0}(K, X)$, por uma aplicação do teorema Hahn-Banach, $\varphi$ se estende a um funcional linear contínuo $\bar{\varphi}$ sobre $\mathcal{M}(K, X)$ de mesma norma. Note-se que ao funcional $\bar{\varphi}$ podemos associar a aplicação $\mu: \mathcal{B}(K) \rightarrow X^{*}$ definida para cada $A \in \mathcal{B}(K)$ e cada $v \in X$ por

$$
\langle\mu(A), v\rangle=\bar{\varphi}\left(\mathbb{1}_{A} \cdot v\right) .
$$

Não é difícil verificar que $\mu$ é uma medida vetorial finitamente aditiva e que satisfaz

$$
\|\varphi\|=|\mu|(K) \quad \text { e } \quad \bar{\varphi}(f)=\int f d \mu, f \in C_{0}(K, X) .
$$

Naturalmente, a extensão $\bar{\varphi}$ não está univocamente determinada assim como a medida $\mu$ correspondente. Para medidas escalares, o clássico Teorema de Representação de Riesz [45, Teorema 6.19] estabelece que o dual de $C_{0}(K)$ pode ser identificado com $\operatorname{rcabv}(K)$ via teoria de integração. Um resultado de I. Singer determina que dentre todas as medidas $\mu$ que cumprem (1.6) existe uma única em $\operatorname{rcabv}\left(K, X^{*}\right)$. Trata-se de uma extensão do Teorema de Representação de Riesz para os espaços $C_{0}(K, X)$.

Teorema 1.17 (Representação de Riesz-Singer). Existe um isomorfismo isométrico entre $C_{0}(K, X)^{*}$ $e \operatorname{rcabv}\left(K, X^{*}\right)$, onde cada funcional $\varphi \in C_{0}(K, X)^{*}$ e a medida $\mu \in \operatorname{rcabv}\left(K, X^{*}\right)$ correspondente se relacionam pela seguinte fórmula integral

$$
\varphi(f)=\int f d \mu, f \in C_{0}(K, X)
$$

com $\|\varphi\|=|\mu|(K)$.

Para a demonstração deste teorema, no caso em que $K$ é compacto, recomendamos [32] e também [48, Lema 1.6, p. 193]. Para $K$ localmente compacto, o teorema pode ser obtido do caso compacto como explicado em [11, p. 2]. Para uma demonstração detalhada do caso localmente compacto recomendamos [40].

Observação 1.18. A demonstração original do Teorema 1.17 descoberta por I. Singer em [49] é incompleta. Este resultado, até onde sabemos, foi completado pela primeira vez por J. Gil de Lamadrid em [30].

\subsection{O Teorema de Radon-Nikodým}

Nesta seção apresentaremos um teorema tipo Radon-Nikodým para medidas vetoriais. Este resultado terá importantes aplicações nos subsequentes capítulos. 
Sejam $(K, \Sigma)$ um espaço mensurável e $X$ um espaço de Banach. Recordamos que uma medida vetorial $\mu: \Sigma \rightarrow X$ é absolutamente contínua com relação a uma medida positiva $\lambda: \Sigma \rightarrow \mathbb{R}$ e escrevemos $\mu \ll \lambda$, se

$$
\lim _{\lambda(A) \rightarrow 0} \mu(A)=0 .
$$

Observação. Devemos notar que $\mu \ll \lambda$ não significa que $\mu(A)=0$ sempre que $\lambda(A)=0$, a menos que $\mu$ e $\lambda$ sejam ambas $\sigma$-aditivas.

Recordamos em seguida um teorema clássico, [45, Teorema 6.10].

Teorema 1.19 (Radon-Nikodým). Sejam $\lambda: \Sigma \rightarrow \mathbb{R}$ uma medida positiva e $\sigma$-aditiva, $\mu: \Sigma \rightarrow \mathbb{K}$ uma medida $\sigma$-aditiva de variação limitada satisfazendo $\mu \ll \lambda$. Então existe uma função $\gamma: K \rightarrow \mathbb{K}$ mensurável, integrável e satisfazendo

$$
\mu(A)=\int_{A} \gamma d \lambda \quad e \quad|\mu|(A)=\int_{A}|\gamma| d \lambda, A \in \Sigma .
$$

Para medidas vetoriais $\mu: \Sigma \rightarrow X$ sabemos que não existem, em geral, densidades $\gamma$ mensuráveis satisfazendo conclusão semelhante a do Teorema 1.19, isto só ocorre quando $X$ tem a propriedade de Radon-Nikodým.

Um espaço de Banach $X$ tem a propriedade de Radon-Nikodým (ou P.R.N.) se para qualquer espaço mensurável $(K, \Sigma)$, qualquer medida positiva e $\sigma$-aditiva $\lambda: \Sigma \rightarrow \mathbb{R}$ e qualquer medida vetorial $\sigma$-aditiva de variação limitada $\mu: \Sigma \rightarrow X$ tal que $\mu \ll \lambda$, existir uma função $\gamma: K \rightarrow X$ mensurável, Bochner integrável e que verifica, para todo $A \in \Sigma$, a relação

$$
\mu(A)=\int_{A} \gamma d \lambda . \text { (Bochner) }
$$

Exemplos de espaços de Banach com essa propriedade são: espaços reflexivos [20, Corolário 13, p. 76] e espaços duais separáveis [20, Teorema 1, p. 79].

Inspirado em [22, Teorema 34, p. 37], apresentaremos uma versão do teorema de Radon-Nikodým para medidas a valores em $X^{*}$ sem a imposição dos espaços envolvidos possuirem a propriedade de Radon-Nikodým. Todavia, a densidade $\gamma$ obtida é apenas $w^{*}$-mensurável.

Definição 1.20. Sejam $(K, \Sigma)$ um espaço mensurável e $X$ um espaço de Banach. Dizemos que uma função $f: K \rightarrow X^{*}$ é $w^{*}$-mensurável se para todo $v \in X$ a função numérica

$$
x \mapsto\langle f(x), v\rangle
$$

for mensurável no sentido usual.

Recordamos que uma propriedade $P(x)$ definida para cada $x \in K$ é dita verdadeira $\mu$-qs ou $\mu$-quase sempre se o conjunto $N=\{x \in K: P(x)$ é falsa $\}$ está contido em algum $A \in \Sigma$ tal que $\mu(A)=0$.

Teorema 1.21. Sejam $K$ um espaço localmente compacto de Hausdorff, $X$ um espaço de Banach separável e $\mu \in \operatorname{rcabv}\left(K, X^{*}\right)$. Existe uma função $\gamma: K \rightarrow X^{*} w^{*}$-mensurável, tal que $\|\gamma(x)\|=1$ para todo $x \in K$ e que verifica

$$
\langle\mu(A), u\rangle=\int_{A}\langle\gamma(x), u\rangle d|\mu|(x), A \in \mathcal{B}(K) \text { e } u \in X .
$$

Demonstração. Para cada $v \in X$ defina a aplicação $\mu_{v}: \mathcal{B}(K) \rightarrow \mathbb{K}$ por

$$
\mu_{v}(A)=\langle\mu(A), v\rangle, A \in \mathcal{B}(K) .
$$


Claramente $\mu_{v} \in \operatorname{rcabv}(K)$ e $\mu_{v} \ll|\mu|$. De acordo com o Teorema de Radon-Nikodým 1.19, existe uma função $\gamma_{v}: K \rightarrow \mathbb{K}$ mensurável, integrável e satisfazendo

$$
\mu_{v}(A)=\int_{A} \gamma_{v} d|\mu| \text { e }\left|\mu_{v}\right|(A)=\int_{A}\left|\gamma_{v}\right| d|\mu|, A \in \mathcal{B}(K) .
$$

Seja $\mathcal{N}(v)=\left\{x \in K:\left|\gamma_{v}\right|(x)>\|v\|\right\}$ e defina para cada $n \in \mathbb{N}$ o conjunto

$$
A_{n}=\left\{x \in K:\left|\gamma_{v}\right|(x) \geq\|v\|+\frac{1}{n}\right\} .
$$

$\operatorname{Temos} \mathcal{N}(v)=\bigcup_{n \in \mathbb{N}} A_{n} \mathrm{e}$

$$
\left(\|v\|+\frac{1}{n}\right)|\mu|\left(A_{n}\right) \leq \int_{A_{n}}\left|\gamma_{v}\right| d|\mu|=\left|\mu_{v}\right|\left(A_{n}\right) \leq\|v\| \cdot|\mu|\left(A_{n}\right), n \in \mathbb{N} .
$$

Então, $|\mu|\left(A_{n}\right)=0$ para todo $n \in \mathbb{N}$ e deduzimos que $|\mu|(\mathcal{N}(v))=0$.

Concluímos que para cada $v \in X$ existe uma função mensurável $\gamma_{v}: K \rightarrow \mathbb{K}$ que verifica (1.7), um boreliano $\mathcal{N}(v)$ com $|\mu|(\mathcal{N}(v))=0$ e vale

$$
\left|\gamma_{v}\right|(x) \leq\|v\|, x \in K \backslash \mathcal{N}(v) .
$$

Para cada $u, v \in X$ e $a, b \in \mathbb{K}$ temos

$$
\mu_{a u+b v}=a \mu_{u}+b \mu_{v}
$$

Então, para cada $A \in \mathcal{B}(K)$ vale a relação

$$
\int_{A} \gamma_{a u+b v} d|\mu|=\mu_{a u+b v}(A)=a \mu_{u}(A)+b \mu_{v}(A)=\int_{A}\left(a \gamma_{u}+b \gamma_{v}\right) d|\mu|
$$

e portanto

$$
\gamma_{a u+b v}(x)=a \gamma_{u}(x)+b \gamma_{u}(x), \mu-q s .
$$

Podemos fixar um boreliano $\mathcal{M}(a, b, u, v)$ tal que $|\mu|(\mathcal{M}(a, b, u, v))=0$ de forma que a relação (1.9) se verifique para todo $x \in K \backslash \mathcal{M}(a, b, u, v)$.

Em seguida, seja $\left\{v_{n}: n \in \mathbb{N}\right\}$ uma sequência densa em $X$. Denote por $\mathbb{K}_{\mathbb{Q}}$ o corpo $\mathbb{Q}$ ou $\mathbb{Q}+i \mathbb{Q}$, conforme $X$ seja um espaço de Banach sobre $\mathbb{R}$ ou $\mathbb{C}$ respectivamente e seja $X_{0}$ o espaço normado gerado sobre $\mathbb{K}_{\mathbb{Q}}$ por $\left\{v_{n}: n \in \mathbb{N}\right\}$. Claramente, $X_{0}$ é enumerável e denso em $X$.

Defina

$$
\mathcal{N}=\bigcup_{\substack{a, b \in \mathbb{K}_{\mathbb{Q}} \\ u, v \in X_{0}}}(\mathcal{N}(a u+b v) \cup \mathcal{M}(a, b, u, v))
$$

e observe que $\mathcal{N} \in \mathcal{B}(K)$ e $|\mu|(\mathcal{N})=0$.

Para cada $v \in X_{0}$ vamos redefinir $\gamma_{v}$ em $\mathcal{N}$ por 0 . Assim, quaisquer que sejam $u, v \in X_{0} \mathrm{e}$ $a, b \in \mathbb{K}_{\mathbb{Q}}$, as relações (1.8) e (1.9) são verdadeiras em todo $K$.

Para cada $x \in K$ considere $\varphi_{x}: X_{0} \rightarrow \mathbb{K}$ por

$$
\varphi_{x}(v)=\gamma_{v}(x), v \in X_{0}
$$

Em virtude de (1.8) e (1.9) serem verdadeiras em todo $K$, a função $\varphi_{x}$ é linear e contínua em $X_{0}$. Portanto, o funcional $\varphi_{x}$ admite uma única extensão de mesma norma a $X$ e que por simplicidade também será denotada por $\varphi_{x}$.

Defina a função $\gamma: K \rightarrow X^{*} \operatorname{como} \gamma(x)=\varphi_{x}, x \in K$. 
Para demonstrar que $\gamma$ é $w^{*}$-mensurável fixe $u \in X$ arbitrário e seja $\left(u_{n}\right)_{n \in \mathbb{N}}$ uma sequência em $X_{0}$ convergindo a $u$. Para cada $n \in \mathbb{N}$, por construção, a aplicação

$$
x \mapsto \varphi_{x}\left(u_{n}\right)=\gamma_{u_{n}}(x)
$$

é mensurável. Consequentemente, a aplicação

$$
x \mapsto\langle\gamma(x), u\rangle=\varphi_{x}(u)=\lim _{n \rightarrow+\infty} \varphi_{x}\left(u_{n}\right)=\lim _{n \rightarrow+\infty} \gamma_{u_{n}}(x)
$$

é mensurável, pois trata-se de um limite pontual de funções mensuráveis.

Em seguida, fixe $A \in \mathcal{B}(K)$ e $u \in X$ arbitrários. Seja $\left(u_{n}\right)_{n \in \mathbb{N}}$ uma sequência em $X_{0}$ convergindo a $u$. Aplicando o Teorema da Convergência Dominada de Lebesgue [45, Teorema 1.34] obtemos

$$
\begin{aligned}
\langle\mu(A), u\rangle & =\lim _{n \rightarrow+\infty}\left\langle\mu(A), u_{n}\right\rangle=\lim _{n \rightarrow+\infty} \mu_{u_{n}}(A)=\lim _{n \rightarrow+\infty} \int_{A} \gamma_{u_{n}}(x) d|\mu|(x) \\
& =\int_{A} \lim _{n \rightarrow+\infty} \gamma_{u_{n}}(x) d|\mu|(x)=\int_{A} \varphi_{x}(u) d|\mu|(x)=\int_{A}\langle\gamma(x), u\rangle d|\mu| .
\end{aligned}
$$

Concluímos que

$$
\langle\mu(A), u\rangle=\int_{A}\langle\gamma(x), u\rangle d|\mu|(x), A \in \mathcal{B}(K) \text { e } u \in X .
$$

Vamos verificar agora que a função $\gamma$ pode ser redefinida em um conjunto de medida nula de modo a satisfazer, além da relação (1.11), $\|\gamma(x)\|=1$ para todo $x \in K$.

Seja $\left\{e_{n}: n \in \mathbb{N}\right\}$ uma sequência densa em $S_{X}$. Por $x \mapsto\left\langle\gamma(x), e_{n}\right\rangle$ ser mensurável para todo $n$, decorre que

$$
x \mapsto\|\gamma(x)\|=\sup _{n \in \mathbb{N}}\left|\left\langle\gamma(x), e_{n}\right\rangle\right|
$$

é mensurável. De acordo com (1.11) temos

$$
\left|\left\langle\mu(A), e_{n}\right\rangle\right|=\left|\int_{A}\left\langle\gamma(x), e_{n}\right\rangle d\right| \mu|(x)| \leq \int_{A}\|\gamma(x)\| d|\mu|(x), A \in \mathcal{B}(K) \text { e } n \in \mathbb{N} .
$$

Deduzimos que

$$
|\mu|(A) \leq \int_{A}\|\gamma(x)\| d|\mu|(x), A \in \mathcal{B}(K) .
$$

Por outro lado, em virtude de $\|\gamma(x)\| \leq 1$ para todo $x \in K$, temos

$$
\int_{A}\|\gamma(x)\| d|\mu|(x) \leq|\mu|(A), \quad A \in \mathcal{B}(K) .
$$

Consequentemente

$$
|\mu|(A)=\int_{A}\|\gamma(x)\| d|\mu|(x), \quad A \in \mathcal{B}(K) .
$$

Por (1.12), inferimos que $\|\gamma(x)\|=1,|\mu|$-qs. Assim, podemos redefinir $\gamma$ em um conjunto de medida nula de modo a satisfazer $\|\gamma(x)\|=1$ para todo $x \in K$.

O Teorema 1.21 é verdadeiro sob condições mais gerais e sem a hipótese de $X$ ser separável. A demonstração envolve o uso de Teoria de Lifting e pode ser encontrada em [20, Teorema 5, p. 269], veja também [15, Teorema 1.5.3, p.22]. Para benefício do leitor enunciaremos, sem demonstração, 
este resultado.

Teorema 1.22. Sejam $(K, \Sigma)$ um espaço mensurável, $\lambda: \Sigma \rightarrow \mathbb{R}$ uma medida positiva, $\sigma$-aditiva e completa, $\mu: \Sigma \rightarrow X^{*}$ uma medida $\sigma$-aditiva e de varição limitada tal que $\mu \ll \lambda$. Então existe uma função $\gamma: K \rightarrow X^{*} w^{*}$-mensurável verificando as seguintes condições:

(a) A função $x \rightarrow\|\gamma(x)\|$ é mensurável e integrável com respeito a $\lambda$.

(b) Para todo $v \in X$ e todo $A \in \Sigma$

$$
\langle\mu(A), v\rangle=\int_{A}\langle\gamma(x), v\rangle d \lambda(x) .
$$

(c) Para todo $A \in \Sigma$

$$
|\mu|(A)=\int_{A}\|\gamma(x)\| d \lambda(x) .
$$

Proposição 1.23. Sejam $K$ um espaço localmente compacto de Hausdorff e $X$ um espaço de Banach. Se $X$ é separável ou $X^{*}$ tem a propriedade de Radon-Nikodým, então para cada $\mu \in$ $\operatorname{rcabv}\left(K, X^{*}\right)$ existe uma função $\gamma: K \rightarrow X^{*} w^{*}$-mensurável verificando as seguintes condições:

(a) $\|\gamma(x)\|=1$ para todo $x \in K$

(b) Para toda $f \in C_{0}(K, X)$, a função $x \mapsto\langle\gamma(x), f(x)\rangle$ é mensurável

(c) Para toda $f \in C_{0}(K, X)$

$$
\int f d \mu=\int\langle\gamma(x), f(x)\rangle d|\mu|(x)
$$

Mais ainda, no caso em que $X^{*}$ tem a propriedade de Radon-Nikodým, $\gamma$ pode ser escolhida mensurável.

Demonstração. Se $X$ for separável, de acordo com o Teorema 1.21, existe uma função $\gamma: K \rightarrow X^{*}$ $w^{*}$-mensurável, tal que $\|\gamma(x)\|=1$ para todo $x \in K$ e verificando

$$
\langle\mu(A), v\rangle=\int_{A}\langle\gamma(x), v\rangle d|\mu|(x), A \in \mathcal{B}(K) \text { e } v \in X
$$

Se $X$ tem a propriedade de Radon-Nikodým, em virtude de $\mu \ll|\mu|$, existe por definição uma função $\gamma: K \rightarrow X^{*}$ mensurável, Bochner integrável e que verifica, para todo $A \in \mathcal{B}(K)$,

$$
\mu(A)=\int_{A} \gamma d|\mu| . \text { (Bochner) }
$$

Observe que a relação (1.13) também é verdadeira neste caso. Mais ainda, de acordo com [20, Teorema 4.(iv), p. 46], vale

$$
|\mu|(A)=\int_{A}\|\gamma(x)\| d|\mu|(x), A \in \mathcal{B}(K)
$$

e decorre que $\|\gamma(x)\|=1,|\mu|$-qs. Assim, podemos redefinir $\gamma$ em um conjunto de medida nula de modo a satifazer, além da relação (1.13), $\|\gamma(x)\|=1$ para todo $x \in K$.

Para ambos os casos, $X$ é separável ou $X^{*}$ tem a propriedade de Radon-Nikodým, seja $f \in$ $C_{0}(K, X)$ arbitrária. De acordo com a Proposição 1.15, podemos fixar uma sequência $\left(f_{n}\right)_{n \in \mathbb{N}}$ em $\mathcal{S}(K, X)$ convergindo uniformemente a $f$ e tal que $\left\|f_{n}\right\| \leq\|f\|$ para todo $n \in \mathbb{N}$. 
Claramente, para todo $n \in \mathbb{N}$, a aplicação $x \mapsto\left\langle\gamma(x), f_{n}(x)\right\rangle$ é mensurável. Consequentemente,

$$
x \mapsto\langle\gamma(x), f(x)\rangle=\lim _{n \rightarrow \infty}\left\langle\gamma(x), f_{n}(x)\right\rangle
$$

é mensurável, pois trata-se de um limite pontual de funções mensuráveis.

Além disso, para todo $x \in K$ e todo $n \in \mathbb{N}$ vale

$$
\left|\left\langle\gamma(x), f_{n}(x)\right\rangle\right| \leq\|\gamma(x)\|\left\|f_{n}(x)\right\| \leq\|f\| .
$$

Aplicando o Teorema da Convergência Dominada de Lebesgue [45, Teorema 1.34] obtemos

$$
\lim _{n \rightarrow \infty} \int\left\langle\gamma(x), f_{n}(x)\right\rangle d|\mu|(x)=\int\langle\gamma(x), f(x)\rangle d|\mu|(x) .
$$

Em seguida, fixe $n \in \mathbb{N}$. Podemos supor que

$$
f_{n}=\sum_{i=1}^{r_{n}} v_{i} \cdot \mathbb{1}_{B_{i}}
$$

onde $B_{1}, \ldots, B_{n}$ é uma partição de $K$ em $\mathcal{B}(K)$. Utilizando a relação (1.13) escrevemos

$$
\begin{aligned}
\int f_{n} d \mu & =\sum_{i=1}^{r_{n}}\left\langle\mu\left(B_{i}\right), v_{i}\right\rangle=\sum_{i=1}^{r_{n}} \int_{B_{i}}\left\langle\gamma(x), v_{i}\right\rangle d|\mu|(x) \\
& =\sum_{i=1}^{r_{n}} \int \mathbb{1}_{B_{i}}(x) \cdot\left\langle\gamma(x), v_{i}\right\rangle d|\mu|(x)=\sum_{i=1}^{r_{n}} \int\left\langle\gamma(x), v_{i} \cdot \mathbb{1}_{B_{i}}(x)\right\rangle d|\mu|(x) \\
& =\int\left\langle\gamma(x), \sum_{i=1}^{r_{n}} v_{i} \cdot \mathbb{1}_{B_{i}}(x)\right\rangle d|\mu|(x)=\int\left\langle\gamma(x), f_{n}(x)\right\rangle d|\mu|(x) .
\end{aligned}
$$

Concluímos que

$$
\int f_{n} d \mu=\int\left\langle\gamma(x), f_{n}(x)\right\rangle d|\mu|(x), n \in \mathbb{N} .
$$

Combinando as relações (1.14) e (1.15) e utilizando a continuidade do funcional linear $f \mapsto \int f d \mu$ obtemos

$$
\int f d \mu=\lim _{n \rightarrow \infty} \int f_{n} d \mu=\lim _{n \rightarrow \infty} \int\left\langle\gamma(x), f_{n}(x)\right\rangle d|\mu|(x)=\int\langle\gamma(x), f(x)\rangle d|\mu|(x) .
$$




\section{Capítulo 2}

\section{Sobre isomorfismos entre espaços de Banach $C_{0}(K, X)$ e a cardinalidade de $K$}

\subsection{Introdução}

Dado um espaço topológico localmente compacto de Hausdorff $K$ e um espaço de Banach $X$ denotamos por $C_{0}(K, X)$ o espaço das funções contínuas de $K$ em $X$ que se anulam no infinito, munido da norma do supremo. Se $X$ for $\mathbb{R}$ ou $\mathbb{C}$, este espaço será denotado por $C_{0}(K)$. Se $K$ for compacto estes espaços serão denotados por $C(K, X)$ e $C(K)$ respectivamente.

Definição 2.1. Sejam $X$ e $Y$ espaços de Banach. Um isomorfismo de $X$ em $Y$ é um operador linear $T: X \rightarrow Y$ contínuo, injetor e com imagem fechada. O número $\|T\|\left\|T^{-1}\right\|$ será denominado distorção de $T$.

Dados espaços de Banach $X$ e $Y$, se existir um isomorfismo sobrejetor de $X$ em $Y$, diremos que $X$ e $Y$ são isomorfos e escreveremos $X \sim Y$. Para enfatizar que existe um isomorfismo sobrejetor com distorção estritamente menor que $\lambda$, para algum $1<\lambda<\infty$, escreveremos $X \stackrel{\lambda}{\sim} Y$. Se existir um isomorfismo sobrejetor $T: X \rightarrow Y$ com distorção $\|T\|\left\|T^{-1}\right\|=1$ diremos que $X$ e $Y$ são isometricamente isomorfos.

Para espaços topológicos compactos de Hausdorff $K_{1}$ e $K_{2}$, o clássico teorema de Banach-Stone afirma que se $C\left(K_{1}\right)$ e $C\left(K_{2}\right)$ forem isometricamente isomorfos, então $K_{1}$ e $K_{2}$ são homeomorfos (mais simplesmente, $K_{1} \approx K_{2}$ ). Este resultado foi obtido por S. Banach [4] para compactos métricos e estendido por M. H. Stone [50] para compactos de Hausdorff. De forma independente, em [3] e [13], este resultado foi generalizado como se segue:

Teorema 2.2 (Amir-Cambern). Sejam $K_{1}$ e $K_{2}$ espaços localmente compactos de Hausdorff. Então

$$
C_{0}\left(K_{1}\right) \stackrel{2}{\sim} C_{0}\left(K_{2}\right) \Longrightarrow K_{1} \approx K_{2}
$$

Quando $K_{1}$ é compacto e $K_{2}$ é não compacto, foi estabelecido por Cambern [9] que 2 é a melhor constante para o teorema acima. Para o caso no qual $K_{1}$ e $K_{2}$ são compactos este fato foi estabelecido por Cohen [18].

Surge em seguida a questão sobre quais relações topológicas são preservadas sob a existência de um isomorfismo sobrejetor $T: C_{0}\left(K_{1}\right) \rightarrow C_{0}\left(K_{2}\right)$ com $\|T\|\left\|T^{-1}\right\| \leq n$. Um fato interessante e não trivial estabelecido por Cengiz [16], sem a hipótese de limitação sobre a distorção, é uma versão fraca do Teorema 2.2 que apresentaremos em seguida. A cardinalidade de um conjunto $K$ será denotada por $|K|$.

Teorema 2.3 (Cengiz). Sejam $K_{1}$ e $K_{2}$ espaços localmente compactos de Hausdorff. Então

$$
C_{0}\left(K_{1}\right) \sim C_{0}\left(K_{2}\right) \Longrightarrow\left|K_{1}\right|=\left|K_{2}\right| \text {. }
$$


O teorema de Banach-Stone foi também generalizado para espaços de funções contínuas assumindo valores em um espaço de Banach. Para espaços de Banach reais, a maior generalização até então é devida a Behrends e Cambern, veja [6]. Para mais generalizações vetoriais do Teorema 2.2 veja também [34].

Teorema 2.4 (Behrends-Cambern). Seja X um espaço de Banach uniformemente não-quadrado. Então existe $1<\lambda \leq 2$ tal que para quaisquer espaços localmente compactos de Hausdorff $K_{1}$ e $K_{2}$,

$$
C_{0}\left(K_{1}, X\right) \stackrel{\lambda}{\sim} C_{0}\left(K_{2}, X\right) \Longrightarrow K_{1} \approx K_{2} .
$$

Recordamos que um espaço de Banach $X$ é uniformemente não-quadrado, ver [33], se existir $0<\delta$ tal que

$$
x, y \in B_{X} \Longrightarrow\left\|\frac{x-y}{2}\right\| \leq 1-\delta \text { ou }\left\|\frac{x+y}{2}\right\| \leq 1-\delta .
$$

Como exemplos de espaços de Banach uniformemente não quadrados destacamos os espaços $l_{p}$, $1<p<\infty$, ver [17]. Por outro lado, é simples verificar que $c_{0}$ e $l_{1}$ não possuem essa propriedade.

Os teoremas 2.3 e 2.4 sugerem, de maneira natural, o seguinte problema:

Problema 2.5. Sejam $X$ um espaço de Banach uniformemente não-quadrado, $K_{1}$ e $K_{2}$ espaços localmente compactos de Hausdorff tais que

$$
C_{0}\left(K_{1}, X\right) \sim C_{0}\left(K_{2}, X\right) .
$$

Nessas condições o que pode ser dito sobre as cardinalidades de $K_{1}$ e $K_{2}$ ?

Neste capítulo daremos uma resposta completa a esta questão. Demonstraremos um resultado mais geral no que diz respeito a isomorfismos entre espaços $C_{0}(K, X)$. Devemos recordar, ver [38] ou [21, p. 218], a seguinte definição:

Definição 2.6. Dizemos que um espaço de Banach $X \neq\{0\}$ tem cotipo finito se existir $2 \leq q<\infty$ e uma constante $\kappa>0$ tal que para todo $n \in \mathbb{N}$ e para toda sequência de vetores $v_{1}, v_{2}, \ldots, v_{n} \in X$, vale

$$
\left(\sum_{i=1}^{n}\left\|v_{i}\right\|^{q}\right)^{\frac{1}{q}} \leq \kappa\left(\int_{0}^{1}\left\|\sum_{i=1}^{n} r_{i}(t) v_{i}\right\|^{2} d t\right)^{\frac{1}{2}},
$$

onde $r_{i}:[0,1] \rightarrow \mathbb{R}$ denotam as funções de Rademacher, definidas por

$$
r_{i}(t)=\operatorname{sign}\left(\sin 2^{i} \pi t\right) .
$$

Observação 2.7. Note-se que cotipo finito é uma propriedade estável sob isomorfismos sobrejetores, i.e., para espaços de Banach $X$ e $Y$, se $X$ tem cotipo finito e $X \sim Y$, então $Y$ tem cotipo finito.

Inspirado por [16] vamos demonstrar a seguinte extensão vetorial do Teorema 2.3.

Teorema 2.8. Seja $X$ um espaço de Banach de cotipo finito. Se $X$ é separável ou se $X^{*}$ tem a propriedade de Radon-Nikodým, então para quaisquer espaços localmente compactos de Hausdorff $K$ e $J$ temos

$$
C_{0}(K, X) \sim C_{0}(J, X) \Longrightarrow|K||J|<\omega_{0} \quad \text { ou } \quad|K|=|J| .
$$

O Teorema 2.8 pode, em um certo sentido, ser considerado uma versão fraca do Teorema 2.4 e fornece imediatamente uma solução para o Problema 2.5. De fato, recorde que um espaço de Banach $X$ é uniformemente convexo, ver [17], se para todo $0<\epsilon \leq 2$ existe $0<\delta$ verificando

$$
x, y \in B_{X} \quad \text { e }\|x-y\| \geq \epsilon \Longrightarrow\left\|\frac{x+y}{2}\right\| \leq 1-\delta .
$$


Por [12, Lema 1] e [21, Teorema 14.1, p. 283], se $X$ é uniformemente convexo então tem cotipo finito. De acordo com um resultado de P. Enflo [24], todo espaço uniformemente não quadrado admite uma norma equivalente uniformemente convexa. Assim, ver Observação 2.7, se $X$ é uniformemente não quadrado então $X$ tem cotipo finito. Um resultado devido a R. C. James [33, Teorema 1.1] afirma que se $X$ é uniformemente não-quadrado então é reflexivo, logo, $X^{*}$ é reflexivo e, segundo um teorema devido a Pettis [20, Corolário 13, p. 76], tem a propriedade de RadonNikodým.

Observação 2.9. Segue diretamente das definições que se $X$ é uniformemente convexo, então $X$ é uniformemente não quadrado. Destacamos que a recíproca não é verdadeira. De fato, considere o espaço

$$
X_{2, \lambda}=\left(l_{2},\|\cdot\|_{2, \lambda}\right), 1<\lambda<\sqrt{2},
$$

onde $\|x\|_{2, \lambda}=\max \left\{\|x\|_{2}, \lambda \cdot\|x\|_{\infty}\right\}$. É fácil verificar que $X_{2, \lambda}$ é um espaço de Banach uniformemente não quadrado. Por outro lado, $X_{2, \lambda}$ não é uniformemente convexo pois existem $x, y \in$ $S_{X_{2, \lambda}}$ tais que

$$
x \neq y \quad \text { e } \quad\left\|\frac{x+y}{2}\right\| \geq 1 .
$$

Note-se que o Teorema 2.8 cobre os casos $l_{1}$ e $X=l_{p}(\Gamma)$, onde $\Gamma$ é um conjunto discreto arbitrário e $1<p<\infty$. Não sabemos responder quando as asserções deste teorema permanecem verdadeiras para o caso remanescente $X=l_{1}(\Gamma)$, onde $\Gamma$ é um conjunto não enumerável.

O clássico teorema de Milyutin [44, Teorema 21.5.10] mostra que não podemos remover a hipótese de cotipo finito no caso em que $X$ é separável. De fato, a conclusão do Teorema 2.8 não vale para uma classe muito grande de espaços de Banach separáveis. Por exemplo, de acordo com [41, Teorema 1], considere a família dos espaços mutuamente não isomorfos $C\left([0,1], l_{p}\right)$ com $1 \leq p<\infty$. Para todo compacto métrico enumerável $K$ temos

$$
C\left(K, C\left([0,1], l_{p}\right)\right) \sim C\left(K \times[0,1], l_{p}\right) \sim C\left([0,1] \times[0,1], l_{p}\right) \sim C\left([0,1], C\left([0,1], l_{p}\right)\right) .
$$

Mais ainda, recordando que para todo conjunto não enumerável $\Gamma$ o espaço $C_{0}(\Gamma)^{*}=l_{1}(\Gamma)$ tem a propriedade de Radon-Nikodým, ver [20, Corolário 8, p. 83], e vale a relação

$$
C_{0}\left(\mathbb{N}, C_{0}(\Gamma)\right) \sim C_{0}(\Gamma) \sim C_{0}\left(\Gamma, C_{0}(\Gamma)\right),
$$

concluímos que a hipótese de cotipo finito no Teorema 2.8 também não pode ser removida no caso em que $X^{*}$ tem a propriedade de Radon-Nikodým.

Organizamos este capítulo do seguinte modo. Na Seção 2.2 vamos estabelecer alguns resultados sobre espaços de Banach de cotipo finito que serão necessários na demonstração do Teorema 2.8. $\mathrm{Na}$ Seção 2.3 demonstraremos um resultado envolvendo funções $w^{*}$-mensuráveis que nos permitirá fornecer uma prova unificada do Teorema 2.8 para os dois casos distintos: $X$ é separável ou $X^{*}$ tem a propriedade de Radon-Nikodým.

Na Seção 2.4 vamos estabelecer um lema envolvendo funções semicontínuas inferiormente associadas a operadores lineares de $C_{0}\left(K_{1}, X\right)$ em $C_{0}\left(K_{2}, X\right)$ e finalmente na Seção 2.5 demonstraremos o Teorema 2.8 .

\subsection{Sobre espaços $C_{0}(K, X), X$ de cotipo finito}

Nesta seção vamos estabelecer alguns resultados auxiliares sobre espaços de Banach de cotipo finito. Um ingrediente fundamental na demonstração destes resultados é o Teorema 1.17 (Representação de Riesz-Singer). 
Proposição 2.10. Sejam $X$ um espaço de Banach de cotipo finito, $K$ e $J$ espaços localmente compactos de Hausdorff. Seja $T$ um isomorfismo de $C_{0}(K, X)$ em $C_{0}(J, X)$ e defina para cada $y \in J$ o conjunto

$$
K_{y}=\left\{x \in K:\left|T^{*}\left(\varphi \cdot \delta_{y}\right)\right|(\{x\})>0 \text { para algum } \varphi \in S_{X^{*}}\right\},
$$

onde $\delta_{y}$ denota a medida de Dirac concentrada em y. Então

(a) $K_{y}$ é enumerável para todo $y \in J$.

(b) Se $J$ é infinito e para cada $x \in K$ existir algum $y \in J$ tal que $x \in K_{y}$, então $|K| \leq|J|$.

Demonstração. (a) Assumiremos que $K_{y}$ é não enumerável para algum $y \in J$ e mostraremos que esta suposição implica uma contradição.

Com efeito, sob esta suposição, existe algum $0<\epsilon<1$ tal que

$$
\left\{x \in K:\left|T^{*}\left(\varphi \cdot \delta_{y}\right)\right|(\{x\})>\epsilon \text { para algum } \varphi \in S_{X^{*}}\right\}
$$

é infinito.

Por $X$ ter cotipo finito $2 \leq q<\infty$, de acordo com a Definição 2.6, existe uma constante $Q>0$ tal que para qualquer sequência de vetores $v_{1}, v_{2}, \ldots, v_{m} \in X$, se $0<\delta \leq\left\|v_{i}\right\|$ para cada $1 \leq i \leq m$, existe uma escolha apropriada de escalares $r_{i}= \pm 1$ verificando

$$
\left\|\sum_{i=1}^{m} r_{i} \cdot v_{i}\right\| \geq \delta \cdot Q \cdot \sqrt[q]{m} .
$$

Fixe $n \in \mathbb{N}$ satisfazendo $\epsilon \cdot Q \cdot \sqrt[q]{n}>2\|T\|$. Fixe também pontos distintos $x_{1}, \ldots, x_{n} \in K$ e $\varphi_{1}, \ldots, \varphi_{n} \in S_{X^{*}}$ tais que

$$
\left\|T^{*}\left(\varphi_{i} \cdot \delta_{y}\right)\left(\left\{x_{i}\right\}\right)\right\|=\left|T^{*}\left(\varphi_{i} \cdot \delta_{y}\right)\right|\left(\left\{x_{i}\right\}\right)>\epsilon, 1 \leq i \leq n .
$$

Existem vetores $v_{1}, \ldots, v_{n}$ na bola unitária de $X$ verificando

$$
\left\langle T^{*}\left(\varphi_{i} \cdot \delta_{y}\right)\left(\left\{x_{i}\right\}\right), v_{i}\right\rangle>\epsilon, 1 \leq i \leq n .
$$

Devido à regularidade das medidas $T^{*}\left(\varphi_{i} \cdot \delta_{y}\right)$ podemos fixar $U_{1}, \ldots, U_{n}$, vizinhanças mutuamente disjuntas de $x_{1}, \ldots, x_{n}$ respectivamente, satisfazendo

$$
\left|T^{*}\left(\varphi_{i} \cdot \delta_{y}\right)\right|\left(U_{i} \backslash\left\{x_{i}\right\}\right) \leq \frac{\epsilon}{2} .
$$

Aplicando o Lema de Urysohn 1.12, podemos encontrar funções $h_{i} \in C_{0}(K)$ com $0 \leq h_{i}(x) \leq 1$ para todo $x \in K, h_{i}\left(x_{i}\right)=1$ e $h_{i}(x)=0$ se $x \in K \backslash U_{i}$. Defina $f_{i} \in C_{0}(K, X)$ por $f_{i}=v_{i} \cdot h_{i}$. Então, das relações (2.2) e (2.3),

$$
\begin{aligned}
\left\|T f_{i}(y)\right\| & \geq\left|\left\langle\varphi_{i}, T f_{i}(y)\right\rangle\right|=\left|\int f_{i} d T^{*}\left(\varphi_{i} \cdot \delta_{y}\right)\right| \\
& \geq\left|\left\langle T^{*}\left(\varphi_{i} \cdot \delta_{y}\right)\left(\left\{x_{i}\right\}\right), v_{i}\right\rangle\right|-\left|\int f_{i} d T^{*}\left(\varphi_{i} \cdot \delta_{y}\right)-\left\langle T^{*}\left(\varphi_{i} \cdot \delta_{y}\right)\left(\left\{x_{i}\right\}\right), v_{i}\right\rangle\right| \\
& >\epsilon-\left|\int f_{i} d T^{*}\left(\varphi_{i} \cdot \delta_{y}\right)-\int_{x_{i}} f_{i} d T^{*}\left(\varphi_{i} \cdot \delta_{y}\right)\right| \\
& \geq \epsilon-\left|T^{*}\left(\varphi_{i} \cdot \delta_{y}\right)\right|\left(U_{i} \backslash\left\{x_{i}\right\}\right) \geq \frac{\epsilon}{2} .
\end{aligned}
$$


De acordo com (2.1) existe uma escolha apropriada de escalares $r_{i}= \pm 1$ tais que

$$
\left\|\sum_{i=1}^{n} r_{i} \cdot T f_{i}(y)\right\| \geq \frac{\epsilon \cdot Q \cdot \sqrt[q]{n}}{2} .
$$

Em virtude de $U_{i} \cap U_{j}=\varnothing$, se $i \neq j$, e $\left\|f_{i}\right\| \leq 1,1 \leq i \leq n$, temos

$$
\left\|\sum_{i=1}^{n} r_{i} \cdot f_{i}\right\| \leq 1
$$

Por (2.4) e pela escolha de $\epsilon$,

$$
\|T\| \geq\left\|T\left(\sum_{i=1}^{n} r_{i} \cdot f_{i}\right)\right\| \geq\left\|T\left(\sum_{i=1}^{n} r_{i} \cdot f_{i}\right)(y)\right\|=\left\|\sum_{i=1}^{n} r_{i} \cdot T f_{i}(y)\right\|>\|T\|,
$$

o que é uma contradição.

(b) Segue de nossa hipótese que

$$
K=\bigcup_{y \in J} K_{y}
$$

De acordo com o item (a), o conjunto $K_{y}$ é enumerável para todo $y \in K$. Portanto $|K| \leq|Y|$.

A soma direta de espaços de Banach $X$ e $Y$ munida da norma do máximo será denotada $X \oplus Y$. Especificamente, $X \oplus Y$ denota o espaço $X \times Y$ munido da norma $\|(x, y)\|=\max \{\|x\|$, $\|y\|\}$. Por questão de simplicidade, denotaremos por $X^{n}$ a soma direta de $n$ cópias de $X$, munida da norma do máximo.

Recordamos o seguinte resultado:

Teorema 2.11 (Samuel). Sejam X e $Y$ espaços de Banach. Então $X$ ou $Y$ contém um subespaço isomorfo a $c_{0}$ se e somente se $X \oplus Y$ contém um subespaço isomorfo a $c_{0}$.

Demonstração. Ver C. Samuel [46, Teorema 1].

Observe que o Teorema 2.8, no caso em que $K$ ou $J$ são finitos, é consequência imediata do seguinte resultado:

Proposição 2.12. Sejam X um espaço de Banach de cotipo finito e $K$ um espaço topológico de Hausdorff finito. Então para todo espaço localmente compacto de Hausdorff $J$ vale

$$
C_{0}(K, X) \sim C_{0}(J, X) \Longrightarrow|J|<\omega_{0} .
$$

Demonstração. Por $K$ ser finito e discreto existe $n \in \mathbb{N}$ tal que

$$
X^{n} \sim C_{0}(K, X) \sim C_{0}(J, X) .
$$

Então $J$ deve ser finito. Do contrário, $C_{0}(J, X)$, e consequentemente $X^{n}$, contém um subespaço isomorfo a $c_{0}$. De acordo com o Teorema $2.11, X$ contém um subespaço isomorfo a $c_{0}$ o que contradiz a hipótese de $X$ ter cotipo finito. 


\subsection{Sobre funções $w^{*}$-mensuráveis}

Nesta seção apresentaremos um resultado envolvendo funções $w^{*}$-mensuráveis que será fundamental na demonstração do principal resultado deste capítulo. Recordamos que dado um espaço mensurável $(K, \Sigma)$ e um espaço de Banach $X$, uma função $\gamma: K \rightarrow X^{*}$ é $w^{*}$-mensurável se para todo $v \in X$ a função numérica $y \mapsto\langle\gamma(y), v\rangle$ for mensurável no sentido usual.

Proposição 2.13. Sejam $X$ um espaço de Banach, J um espaço localmente compacto de Hausdorff, $\mu \in \operatorname{rcabv}(J)$ e $\gamma: J \rightarrow X^{*}$ uma função tal que $\|\gamma(y)\|=1$ para todo $y \in J$. Com relação à $\sigma$-álgebra de Borel de J, suponha que

(a) X é separável e $\gamma$ é $w^{*}$-mensurável ou

(b) $\gamma$ é mensurável.

Então para cada $\epsilon>0$ existe um compacto $J_{0} \subset J$ tal que $|\mu|\left(J \backslash J_{0}\right) \leq \epsilon$ e para cada $v \in X$ a restrição da função numérica $y \mapsto\langle\gamma(y), v\rangle$ a $J_{0}$ é contínua.

Demonstração. (a) Considere a esfera $S_{X^{*}}$ munida da topologia fraca*. Seja $\left\{v_{n}: n \in \mathbb{N}\right\}$ uma sequência densa em $S_{X^{*}}$ e defina

$$
\mathrm{d}(\psi, \varphi)=\sum_{n=1}^{\infty} \frac{1}{2^{n}}\left|\left\langle\psi, v_{n}\right\rangle-\left\langle\varphi, v_{n}\right\rangle\right|, \quad \psi, \varphi \in S_{X^{*}}
$$

Demonstra-se que d é uma métrica que induz a topologia de $S_{X^{*}}$.

Fixe $\epsilon>0$ arbitrário. Para cada $n \in \mathbb{N}$, por $\gamma(J) \subseteq S_{X^{*}}$ e por $S_{X^{*}}$ ser compacto na topologia fraca* $^{*}$, ver [26, Teorema 3.37, p. 99], podemos recobrir $\gamma(J)$ com uma coleção finita de bolas abertas $\left\{U_{1}, \ldots, U_{r_{n}}\right\}$ de raio $\frac{1}{2 n}$, onde $r_{n}$ é o menor inteiro positivo para o qual existe tal coleção.

Para cada $1 \leq i \leq r_{n}$ suponha

$$
U_{i}=\left\{\psi \in S_{X^{*}}: \mathrm{d}\left(\psi, \varphi_{i}\right)<\frac{1}{2 n}\right\}
$$

e considere a função $\eta_{i}: J \rightarrow \mathbb{R}$ definida por

$$
\eta_{i}(y)=\mathrm{d}\left(\gamma(y), \varphi_{i}\right), y \in J .
$$

Por $\gamma$ ser $w^{*}$-mensurável, $\eta_{i}$ é mensurável pois se trata de um limite pontual de funções mensuráveis. Mais ainda, por

$$
\eta_{i}^{-1}\left(\left[0, \frac{1}{2 n}[)=\gamma^{-1}\left(U_{i}\right),\right.\right.
$$

deduzimos que $\gamma^{-1}\left(U_{i}\right)$ é um boreliano para todo $1 \leq i \leq r_{n}$. Então, se

$$
A_{i}=U_{i} \backslash \bigcup_{j<i} U_{j} \quad \text { e } \quad B_{i}=\gamma^{-1}\left(A_{i}\right), 1 \leq i \leq r_{n},
$$

podemos concluir que $B_{1}, \ldots, B_{r_{n}}$ são borelianos mutuamente disjuntos satisfazendo

$$
J=\bigcup_{1 \leq i \leq r_{n}} B_{i}
$$

Devido à regularidade de $|\mu|$ existe para cada $1 \leq i \leq r_{n}$, um compacto não vazio $L_{i} \subset B_{i}$ satisfazendo

$$
|\mu|\left(B_{i} \backslash L_{i}\right) \leq \frac{\epsilon}{r_{n} \cdot 2^{n}}
$$


Para cada $1 \leq i \leq r_{n}$ fixe $y_{i} \in L_{i}$ e defina

$$
\gamma_{n}=\sum_{i=1}^{r_{n}} \gamma\left(y_{i}\right) \cdot \mathbb{1}_{L_{i}}
$$

Em virtude de $L_{1}, \ldots, L_{r_{n}}$ serem mutuamente disjuntos, se $y \in L_{1} \cup \ldots \cup L_{r_{n}}$ então

$$
\mathrm{d}\left(\gamma(y), \gamma_{n}(y)\right)<\frac{1}{n} .
$$

Para cada $n \in \mathbb{N}$ defina $K_{n}=L_{1} \cup \ldots \cup L_{r_{n}}$. Observe que

$$
|\mu|\left(J \backslash K_{n}\right)=|\mu|\left(\left(\bigcup_{1 \leq i \leq r_{n}} B_{i}\right) \backslash K_{n}\right)=\sum_{i=1}^{r_{n}}|\mu|\left(B_{i} \backslash L_{i}\right) \leq \frac{\epsilon}{2^{n}}
$$

e que a restrição de $\gamma_{n}$ a $K_{n}$ é contínua.

Defina $J_{0}=\bigcap_{n=1}^{\infty} K_{n}$. Temos

$$
|\mu|\left(J \backslash J_{0}\right)=|\mu|\left(\bigcup_{n=1}^{\infty}\left(J \backslash K_{n}\right)\right) \leq \sum_{n=1}^{\infty}|\mu|\left(J \backslash K_{n}\right) \leq \epsilon .
$$

Por construção, a sequência $\left(\gamma_{n}\right)_{n \in \mathbb{N}}$, restrita a $J_{0}$, converge uniformemente, na métrica d, para a restrição de $\gamma$ a $J_{0}$. Assim, considerando sobre $X^{*}$ a topologia fraca*, a restrição de $\gamma$ a $J_{0}$ é contínua. De forma equivalente, para cada $v \in X$, a restrição da função numérica $y \mapsto\langle\gamma(y), v\rangle$ a $J_{0}$ é contínua.

(b) Se a função $\gamma$ é mensurável, então existe uma sequência de funções simples $\left(\gamma_{n}\right)_{n \in \mathbb{N}}$ convergindo pontualmente a $\gamma$. Dado $\epsilon>0$, o Teorema de Egorov [22, Teorema 42, p. 18] fornece um conjunto mensurável $N \subset J$ tal que $|\mu|(N) \leq \frac{\epsilon}{2}$ e a sequência $\left(\gamma_{n}\right)_{n \in \mathbb{N}}$ converge a $\gamma$ uniformemente sobre $J \backslash N$, na métrica induzida pela norma.

Dado $n \in \mathbb{N}$ suponha

$$
\gamma_{n}=\sum_{i=1}^{r_{n}} v_{i} \cdot \mathbb{1}_{B_{i}}
$$

onde $v_{1}, \ldots, v_{r_{n}}$ são vetores em $X$ e $B_{1}, \ldots, B_{r_{n}}$ são borelianos não vazios, mutuamente disjuntos e verificam

$$
J=\bigcup_{1 \leq i \leq r_{n}} B_{i}
$$

Devido à regularidade de $|\mu|$ podemos encontrar para cada $1 \leq i \leq r_{n}$, um compacto $L_{i} \subset B_{i} \backslash N$ verificando

$$
|\mu|\left(\left(B_{i} \backslash N\right) \backslash L_{i}\right) \leq \frac{\epsilon}{r_{n} \cdot 2^{n+1}} .
$$

Defina $K_{n}=L_{1} \cup \ldots \cup L_{r_{n}}, n \in \mathbb{N}$. Temos

$$
|\mu|\left((J \backslash N) \backslash K_{n}\right)=|\mu|\left(\left(\bigcup_{1 \leq i \leq r_{n}}\left(B_{i} \backslash N\right)\right) \backslash K_{n}\right)=\sum_{i=1}^{r_{n}}|\mu|\left(\left(B_{i} \backslash N\right) \backslash L_{i}\right) \leq \frac{\epsilon}{2^{n+1}}
$$

e claramente a restrição de $\gamma_{n}$ a $K_{n}$ é contínua. 
Defina $J_{0}=\bigcap_{n=1}^{\infty} K_{n}$. Então

$$
|\mu|\left((J \backslash N) \backslash J_{0}\right)=|\mu|\left(\bigcup_{n=1}^{\infty}\left((J \backslash N) \backslash K_{n}\right)\right) \leq \sum_{n=1}^{\infty}|\mu|\left((J \backslash N) \backslash K_{n}\right) \leq \frac{\epsilon}{2} .
$$

Resulta que

$$
|\mu|\left(J \backslash J_{0}\right)=|\mu|\left((J \backslash N) \backslash J_{0}\right)+|\mu|\left(N \backslash J_{0}\right) \leq \epsilon .
$$

Por construção, a sequência $\left(\gamma_{n}\right)_{n \in \mathbb{N}}$, restrita ao compacto $J_{0}$, converge uniformemente, na métrica induzida pela norma, para a restrição da função $\gamma$ a $J_{0}$, logo, a restrição de $\gamma$ a $J_{0}$ é contínua. Concluímos que para cada $v \in X$, a restrição da função numérica $y \mapsto\langle\gamma(y), v\rangle$ a $J_{0}$ é contínua.

\subsection{Sobre funções semicontínuas inferiormente}

Nesta seção apresentaremos um resultado envolvendo funções semicontínuas inferiormente que será utilizado posteriormente. Recordamos que uma função $f$ a valores reais é denominada semicontínua inferiormente se o conjunto $f^{-1}(] r,+\infty[)$ for aberto para todo $r \in \mathbb{R}$, ver [47, Definição 6.3.1].

Lema 2.14. Sejam $X$ um espaço de Banach, $K$ e $J$ espaços localmente compactos de Hausdorff, $S$ um operador linear contínuo de $C_{0}(K, X)$ em $C_{0}(J, X)$ e $\gamma$ uma função de $J$ em $X^{*}$ satisfazendo $\|\gamma(y)\|=1$ para todo $y \in J$ e tal que para todo $v \in X$ a função numérica $y \mapsto\langle\gamma(y)$, $v\rangle$ é contínua. Então para cada aberto $U \subset K$ a função

$$
y \longmapsto\left|S^{*}\left(\gamma(y) \cdot \delta_{y}\right)\right|(U)
$$

é semicontínua inferiormente.

Demonstração. De início, observe que para cada $f \in C_{0}(K, X)$ a função

$$
y \longmapsto\left|\int f d S^{*}\left(\gamma(y) \cdot \delta_{y}\right)\right|=|\langle\gamma(y), S f(y)\rangle|
$$

é contínua. De fato, dado $f \in C_{0}(K, X)$ fixe $y_{0} \in J$. Em virtude de $\|\gamma(y)\|=1$ para todo $y \in J$, vale

$$
\begin{aligned}
\left|\langle\gamma(y), S f(y)\rangle-\left\langle\gamma\left(y_{0}\right), S f\left(y_{0}\right)\right\rangle\right|= & \left|\left\langle\gamma(y), S f(y)-S f\left(y_{0}\right)\right\rangle+\left\langle\gamma(y)-\gamma\left(y_{0}\right), S f\left(y_{0}\right)\right\rangle\right| \\
& \leq\left\|S f(y)-S f\left(y_{0}\right)\right\|+\left|\left\langle\gamma(y)-\gamma\left(y_{0}\right), S f\left(y_{0}\right)\right\rangle\right|
\end{aligned}
$$

A relação acima e a continuidade das funções $y \mapsto\left\langle\gamma(y), S f\left(y_{0}\right)\right\rangle$ e $S f: J \rightarrow X$ implicam a continuidade da função $y \mapsto|\langle\gamma(y), S f(y)\rangle|$.

Em seguida, dado um aberto $U \subset K$, considere a coleção

$$
\mathcal{F}_{U}=\left\{f \in C_{0}(K, X):\|f\| \leq 1 \text { e } f(x)=0 \text { para todo } x \in K \backslash U\right\} .
$$

Como visto acima, $y \mapsto|\langle\gamma(y), S f(y)\rangle|$ é contínua para todo $f \in \mathcal{F}_{U}$. Portanto

$$
y \longmapsto \sup _{\mathcal{F}_{U}}\left|\int f d S^{*}\left(\gamma(y) \cdot \delta_{y}\right)\right|
$$

é semicontínua inferiormente. 
Por uma aplicação do Teorema 1.16 concluímos que a função

$$
y \longmapsto \sup _{\mathcal{F}_{U}}\left|\int f d S^{*}\left(\gamma(y) \cdot \delta_{y}\right)\right|=\left|S^{*}\left(\gamma(y) \cdot \delta_{y}\right)\right|(U)
$$

é semicontínua inferiormente.

\subsection{Demonstração do Teorema 2.8}

Finalmente, demonstraremos o principal resultado deste capítulo.

Demonstração do Teorema 2.8. Podemos assumir, de acordo com a Proposição 2.12, que $K$ e $J$ são ambos infinitos. Demonstraremos que $|K| \leq|J|$ e então, devido à simetria dos argumentos, também teremos $|J| \leq|K|$.

Vamos aplicar o item (b) da Proposição 2.10 para provar que $|K| \leq|J|$. Bastará estabelecer que para cada $x \in K$ existe algum $y \in J$ tal que $x \in K_{y}$.

Por contradição, suponha existir $x \in K$ tal que para cada $y \in J$ e para cada $\varphi \in S_{X^{*}}$

$$
\left|T^{*}\left(\varphi \cdot \delta_{y}\right)\right|(\{x\})=0 .
$$

Fixe $\Phi \in S_{X^{*}}$ e considere a medida vetorial $\mu_{x} \in \operatorname{rcabv}\left(J, X^{*}\right)$ definida por

$$
\mu_{x}=\left(T^{-1}\right)^{*}\left(\Phi \cdot \delta_{x}\right) .
$$

De acordo com a Proposição 1.23 existe uma função $w^{*}$-mensurável $\gamma: J \rightarrow X^{*}$, que é mensurável quando $X^{*}$ tem a propriedade de Radon-Nikodým, tal que $\|\gamma(y)\|=1$ para todo $y \in J$ e que verifica a relação

$$
\int f d \mu_{x}=\int\langle\gamma(y), f(y)\rangle d\left|\mu_{x}\right|(y), f \in C_{0}(J, X) .
$$

Em seguida, fixe $\epsilon>0$ satisfazendo

$$
\epsilon<\min \left\{\left|\mu_{x}\right|(J), \frac{1}{2\left(\|T\|+\left|\mu_{x}\right|(J)\right)}\right\} .
$$

Aplicando a Proposição 2.13, obtemos um compacto $J_{0} \subseteq J$ tal que

$$
\left|\mu_{x}\right|\left(J \backslash J_{0}\right) \leq \epsilon
$$

e para cada $v \in X$ a restrição da função numérica $y \mapsto\langle\gamma(y), v\rangle$ a $J_{0}$ é contínua.

Para cada $y \in J$ defina

$$
\Gamma_{y}=\left|T^{*}\left(\gamma(y) \cdot \delta_{y}\right)\right| .
$$

Por nossa suposição em (2.5) temos $\Gamma_{y}(\{x\})=0$ para todo $y \in J$.

Seja $\left\{U_{i}: i \in I\right\}$ a coleção de todas as vizinhanças abertas de $x$, dirigida sob a ordem parcial da inclusão reversa, i.e., $i \leq j$ se e somente se $U_{j} \subseteq U_{i}$. Defina para cada $i \in I$ o seguinte conjunto mensurável:

$$
C_{i}=\overline{\left\{y \in J_{0}: \Gamma_{y}\left(\overline{U_{i}}\right) \leq \epsilon\right\}} .
$$

Em virtude de $\Gamma_{y}(\{x\})=0$ para todo $y \in J_{0}$ e devido à regularidade de cada uma das medidas $\Gamma_{y}$ temos

$$
J_{0}=\bigcup_{i \in I} C_{i}
$$


Para completar a demonstração do Teorema 2.8 precisamos verificar duas afirmações referentes às medidas $\Gamma_{y}$ e $\mu_{x}$.

Afirmação 1. $\Gamma_{y}(K)=\sup _{i \in I} \Gamma_{y}\left(K \backslash \overline{U_{i}}\right), y \in J$.

Claramente $\sup _{i \in I} \Gamma_{y}\left(K \backslash \overline{U_{i}}\right) \leq \Gamma_{y}(K)$. Precisamos apenas verificar a desigualdade contrária.

Fixe $y \in J$. Em virtude de $\Gamma_{y}(\{x\})=0$ vale $\Gamma_{y}(K \backslash\{x\})=\Gamma_{y}(K)$. Dado $\delta>0$ arbitrário, devido à regularidade de $\Gamma_{y}$, existe um compacto $K_{0} \subset K \backslash\{x\}$ que verifica

$$
\Gamma_{y}(K)<\delta+\Gamma_{y}\left(K_{0}\right)
$$

Por $K$ ser um espaço localmente compacto de Hausdorff, existe algum aberto $V$ de fecho compacto tal que

$$
K_{0} \subset V \subset \bar{V} \subset K \backslash\{x\} .
$$

Por $\left\{U_{i}\right\}_{i \in I}$ consistir de todas as vizinhanças abertas de $x$. Existe $i_{0} \in I$ tal que $U_{i_{0}}=K \backslash \bar{V}$. Temos $K_{0} \subset K \backslash \overline{K \backslash \bar{V}}=K \backslash \overline{U_{i_{0}}}$ e portanto

$$
\sup _{i \in I} \Gamma_{y}\left(K \backslash \overline{U_{i}}\right) \leq \Gamma_{y}(K)<\delta+\Gamma_{y}\left(K_{0}\right) \leq \delta+\Gamma_{y}\left(K \backslash \overline{U_{i_{0}}}\right) \leq \delta+\sup _{i \in I} \Gamma_{y}\left(K \backslash \overline{U_{i}}\right) .
$$

Por $\delta>0$ ser arbitrário, deduzimos que

$$
\Gamma_{y}(K)=\sup _{i \in I} \Gamma_{y}\left(K \backslash \overline{U_{i}}\right) .
$$

Isso estabelece a Afirmação 1.

Afirmação 2. $\left|\mu_{x}\right|\left(J_{0}\right)=\sup _{i \in I}\left|\mu_{x}\right|\left(C_{i}\right)$.

Observe que a coleção $\left\{C_{i}\right\}_{I}$ é dirigida sob ordem parcial da inclusão direta, i.e., $i \leq j$ se e somente se $C_{i} \subseteq C_{j}$. Então é possível obter uma sequência $i_{1} \leq i_{2} \leq i_{3} \leq \ldots$ satisfazendo

$$
C_{i_{1}} \subseteq C_{i_{2}} \subseteq C_{i_{3}} \ldots \quad \text { e } \quad \lim _{n \rightarrow \infty}\left|\mu_{x}\right|\left(C_{i_{n}}\right)=\sup _{i \in I}\left|\mu_{x}\right|\left(C_{i}\right) .
$$

Seja $C=\bigcup_{n=1}^{\infty} C_{i_{n}}$. As relações em (2.8) implicam

$$
\left|\mu_{x}\right|(C)=\lim _{n \rightarrow \infty}\left|\mu_{x}\right|\left(C_{i_{n}}\right)=\sup _{i \in I}\left|\mu_{x}\right|\left(C_{i}\right) .
$$

Para estabelecer a Afirmação 2 demonstraremos que $\left|\mu_{x}\right|\left(J_{0}\right)=\left|\mu_{x}\right|(C)$. É suficiente, tendo em vista a regularidade $\left|\mu_{x}\right|$, verificar que $\left|\mu_{x}\right|(L)=0$ para qualquer compacto $L \subseteq J_{0} \backslash C$. É útil observar que para qualquer compacto $L \subseteq J_{0} \backslash C$ vale

$$
\left|\mu_{x}\right|\left(C_{i} \cap L\right)=0, i \in I .
$$

De fato, dado $i \in I$ seja $n \in \mathbb{N}$ arbitrário. Existe $j \in I$ tal que $i \leq j$ e $i_{n} \leq j$. Então

$$
\begin{aligned}
\left|\mu_{x}\right|(C) & \geq\left|\mu_{x}\right|\left(C_{j}\right)=\left|\mu_{x}\right|\left(C_{j} \cap L\right)+\left|\mu_{x}\right|\left(C_{j} \cap\left(J_{0} \backslash L\right)\right) \\
& \geq\left|\mu_{x}\right|\left(C_{i} \cap L\right)+\left|\mu_{x}\right|\left(C_{i_{n}}\right),
\end{aligned}
$$

pois $C_{i_{n}} \subset C_{j} \cap\left(J_{0} \backslash L\right)$. Passando-se ao limite na expressão acima para $n$ tendendo ao infinito obtemos $\left|\mu_{x}\right|\left(C_{i} \cap L\right)=0$. 
Seja $L \subseteq J_{0} \backslash C$ um compacto e suponha $\left|\mu_{x}\right|(L)>0$. Defina

$$
M=\sup _{y \in L} \Gamma_{y}(K) .
$$

Temos $M \leq\|T\|<\infty$, pois para cada $y \in L$ vale

$$
\Gamma_{y}(K)=\left|T^{*}\left(\gamma(y) \cdot \delta_{y}\right)\right|(K)=\left\|T^{*}\left(\gamma(y) \cdot \delta_{y}\right)\right\| \leq\|T\| .
$$

Seja $R: C_{0}(J, X) \rightarrow C\left(J_{0}, X\right)$ a restrição natural a $J_{0}$ e considere o operador linear $S=R \circ T:$ $C_{0}(K, X) \rightarrow C\left(J_{0}, X\right)$. Recorde que $\|\gamma(y)\|=1$ para todo $y \in J_{0}$ e para todo $v \in X$ a função $y \mapsto\langle\gamma(y), v\rangle$ é contínua em $J_{0}$. Aplicando o Lema 2.14, deduzimos que para todo conjunto aberto $U \subset K$, a função

$$
y \longmapsto\left|S^{*}\left(\gamma(y) \cdot \delta_{y}\right)\right|(U)=\left|T^{*}\left(\gamma(y) \cdot \delta_{y}\right)\right|(U)=\Gamma_{y}(U),
$$

definida em $J_{0}$, é semicontínua inferiormente. Então, para cada $i \in I$ o conjunto

$$
A_{i}=\left\{y \in L: \Gamma_{y}\left(K \backslash \overline{U_{i}}\right)>M-\epsilon\right\}
$$

é aberto em $L$. Mais ainda, para cada $y \in A_{i}$ temos

$$
\Gamma_{y}(K)=\Gamma_{y}\left(K \backslash \overline{U_{i}}\right)+\Gamma_{y}\left(\overline{U_{i}}\right)>M-\epsilon+\Gamma_{y}\left(\overline{U_{i}}\right) .
$$

Consequentemente,

$$
\Gamma_{y}\left(\overline{U_{i}}\right)<\epsilon-M+\Gamma_{y}(K) \leq \epsilon .
$$

Concluímos que $A_{i} \subset L \cap C_{i}, i \in I$, e então, por consequência de (2.9), $\left|\mu_{x}\right|\left(A_{i}\right)=0, i \in I$.

Seja $y \in L$ tal que $\Gamma_{y}(K)>M-\epsilon$. De acordo com a Afirmação 1 existe $i_{0} \in I$ tal que $\Gamma_{y}\left(K \backslash \overline{U_{i_{0}}}\right)>M-\epsilon$. Então $y \in A_{i_{0}}$ e segue que $F_{0}=L \backslash A_{i_{0}}$ é um subconjunto fechado e próprio de $L$ satisfazendo

$$
\left|\mu_{x}\right|\left(F_{0}\right)=\left|\mu_{x}\right|(L) .
$$

Seja $\mathcal{G}$ a coleção de todos os subconjuntos fechados próprios $F \subset L$ com $\mu(F)=\mu(L)$. Tal coleção, como acabamos de ver, é não vazia. Mais ainda, a relação $F_{1} \leq F_{2}$ se e somente se $F_{2} \subseteq F_{1}$ é uma ordem parcial sobre $\mathcal{G}$.

Fixe $\mathcal{C}=\left\{F_{i}: i \in \Lambda\right\}$ um subconjunto totalmente ordenado de $\mathcal{G}$. Observe que $\mathcal{C}$ é uma coleção de fechados no compacto $L$ com a propriedade da interseção finita e portanto

$$
F=\bigcap_{i \in \Lambda} F_{i} \neq \varnothing
$$

Se $\left|\mu_{x}\right|(F)<\left|\mu_{x}\right|(L)$, então devido à regularidade de $\left|\mu_{x}\right|$, existe $U \subset L$, aberto em $L$, contendo $F$ e verificando

$$
\left|\mu_{x}\right|(F) \leq\left|\mu_{x}\right|(U)<\left|\mu_{x}\right|(L)
$$

Dessa forma, $\left\{F_{i} \backslash U: i \in \Lambda_{0}\right\}$ é uma coleção de subconjuntos fechados de $L$ com a propriedade da interseção finita e com interseção vazia. Isto contradiz o fato de $L$ ser compacto. Então, $\left|\mu_{x}\right|(F)=$ $\left|\mu_{x}\right|(L)$ e $F$ é uma cota superior de $\mathcal{C}$ em $\mathcal{G}$.

Concluímos que todo subconjunto totalmente ordenado de $\mathcal{G}$ tem cota superior em $\mathcal{G}$. De acordo com o Lema de Zorn [47, p. 36], existe em $\mathcal{G}$ um elemento maximal $F_{\text {max }}$.

Repetindo todo o processo acima, desta vez com

$$
M=\sup _{y \in F_{\max }} \Gamma_{y}(K) \text { e } A_{i}=\left\{y \in F_{\max }: \Gamma_{y}\left(K \backslash \overline{U_{i}}\right)>M-\epsilon\right\}
$$


é possível obter um subconjunto fechado próprio $F_{0} \subset F_{\max }$ verificando

$$
\left|\mu_{x}\right|\left(F_{0}\right)=\left|\mu_{x}\right|\left(F_{\max }\right)=\left|\mu_{x}\right|(L),
$$

o que contradiz a maximalidade de $F_{\max }$. Resulta que $\left|\mu_{x}\right|(L)=0$ e isso estabelece a Afirmação 2 .

Vamos agora retomar a demonstração do Teorema 2.8.

Seja $v \in X$ tal que $\|v\|=1$ e $\frac{1}{2}<\langle\Phi, v\rangle$. Aplicando o Lema de Urysohn 1.12, podemos fixar funções $h_{i} \in C_{0}(K), i \in I$, tais que $0 \leq h_{i} \leq 1, h_{i}(x)=1$ e $h_{i}(z)=0$ se $z \in K \backslash U_{i}$. Defina $f_{i} \in C_{0}(K, X)$ por $f_{i}=v \cdot h_{i}$. Recordando a definição de $\mu_{x}$ escrevemos

$$
\frac{1}{2}<\left\langle\Phi, f_{i}(x)\right\rangle=\int f_{i} d\left(\Phi \cdot \delta_{x}\right)=\int T f_{i} d\left(\left(T^{-1}\right)^{*}\left(\Phi \cdot \delta_{x}\right)\right)=\int T f_{i} d \mu_{x} .
$$

Então, de acordo com a relação (2.6), vale

$$
\frac{1}{2}<\int T f_{i} d \mu_{x}=\int\left\langle\gamma(y), T f_{i}(y)\right\rangle d\left|\mu_{x}\right|(y), i \in I .
$$

Vamos dividir esta integral em três partes. Por $\|\gamma(y)\|=1$ para todo $y \in J$ e por (2.7) deduzimos

$$
\left|\int_{J \backslash J_{0}}\left\langle\gamma(y), T f_{i}(y)\right\rangle d\right| \mu_{x}|(y)||\leq\|T\|| \mu_{x} \mid\left(J \backslash J_{0}\right) \leq\|T\| \epsilon .
$$

Por outro lado, para cada $y \in J_{0}$ e $i \in I$,

$$
\left|\left\langle\gamma(y), T f_{i}(y)\right\rangle\right|=\left|\int T f_{i} d \gamma(y) \cdot \delta_{y}\right|=\left|\int f_{i} d T^{*}\left(\gamma(y) \cdot \delta_{y}\right)\right| \leq \Gamma_{y}\left(\overline{U_{i}}\right) .
$$

Recorde que para cada $v \in X$, a restrição da função $y \mapsto\langle\gamma(y), v\rangle$ a $J_{0}$ é contínua e que $\|\gamma(y)\|=1$ para cada $y \in J$. Demonstra-se, como no Lema 2.14, que $y \mapsto\left|\left\langle\gamma(y), T f_{i}(y)\right\rangle\right|, i \in I$, é contínua em $J_{0}$. Então, sempre que $y \in C_{i}$, ver (2.12), temos $\left|\left\langle\gamma(y), T f_{i}(y)\right\rangle\right| \leq \epsilon$ e podemos escrever

$$
\left|\int_{C_{i}}\left\langle\gamma(y), T f_{i}(y)\right\rangle d\right| \mu_{x}|(y)| \leq \int_{C_{i}}\left|\left\langle\gamma(y), T f_{i}(y)\right\rangle\right| d\left|\mu_{x}\right|(y) \leq \epsilon\left|\mu_{x}\right|(J) .
$$

Reunindo as relações (2.11), (2.13) e utilizando novamente o fato de $\|\gamma(y)\|=1$ para todo $y \in J$ temos para cada $i \in I$,

$$
\begin{aligned}
\int\left\langle\gamma(y), T f_{i}(y)\right\rangle d\left|\mu_{x}\right|(y) & =\int_{J \backslash J_{0}}+\int_{J_{0} \backslash C_{i}}+\int_{C_{i}}\left\langle\gamma(y), T f_{i}(y)\right\rangle d\left|\mu_{x}\right|(y) \\
& \leq \epsilon\|T\|+\left|\int_{J_{0} \backslash C_{i}}\left\langle\gamma(y), T f_{i}(y)\right\rangle d\right| \mu_{x}|(y)|+\epsilon\left|\mu_{x}\right|(J) \\
& \leq \epsilon\left(\|T\|+\left|\mu_{x}\right|(J)\right)+\|T\|\left(\left|\mu_{x}\right|\left(J_{0}\right)-\left|\mu_{x}\right|\left(C_{i}\right)\right) .
\end{aligned}
$$

Finalmente, combinando as relações (2.10),(2.14) e utilizando a Afirmação 2, concluímos que

$$
\frac{1}{2} \leq \epsilon\left(\|T\|+\left|\mu_{x}\right|(J)\right) .
$$

Isto contradiz a escolha de $\epsilon$. 


\section{Capítulo 3}

\section{Sobre isomorfismos entre espaços de Banach $C(K, X)$ com distorção menor que 3}

\subsection{Introdução}

Retomando o resultado de Amir e Cambern, o Teorema 2.2 do capítulo anterior, no caso particular onde $K_{1}$ e $K_{2}$ são espaços compactos, por desconhecer isomorfismos sobrejetores $T$ : $C\left(K_{1}\right) \rightarrow C\left(K_{2}\right)$ com $2 \leq\|T\|\left\|T^{-1}\right\|<3$, Amir conjecturou que $K_{1}$ e $K_{2}$ seriam homeomorfos se $\|T\|\left\|T^{-1}\right\|<3$. Mais precisamente, o número 2 poderia substituído por 3 no Teorema 2.2, ver [3]. Reforçando esta conjectura, ver [31], Gordon estabeleceu:

Teorema 3.1 (Gordon). Sejam $K_{1}$ e $K_{2}$ espaços compactos métricos enumeráveis. Então

$$
C\left(K_{1}\right) \stackrel{3}{\sim} C\left(K_{2}\right) \Longrightarrow K_{1} \approx K_{2} .
$$

Entretanto, Cohen [18] provou ser falsa a conjectura de Amir para a classe dos espaços compactos métricos não enumeráveis. Em outras palavras, existem compactos métricos $K_{1}$ e $K_{2}$ não homeomorfos e um isomorfismo sobrejetor $T: C\left(K_{1}\right) \rightarrow C\left(K_{2}\right)$ com $\|T\|\left\|T^{-1}\right\|=2$. Para a construção de diversos exemplos veja [19].

Inspirado no trabalho de Gordon [31], buscamos neste capítulo extensões em $C(K, X)$, onde $K$ é um compacto homeomorfo a um intervalo de ordinal e $X$ é um espaço de Banach, para os teoremas de Amir-Cambern 2.2, Gordon 3.1 e Behrends-Cambern 2.4. Estes resultados sugerem a seguinte pergunta natural:

Problema 3.2. Sejam $K_{1}$ e $K_{2}$ espaços compactos métricos enumeráveis. Suponha existir um espaço de Banach $X$ de cotipo finito, tal que

$$
C\left(K_{1}, X\right) \stackrel{3}{\sim} C\left(K_{2}, X\right)
$$

Nestas condições, o que pode ser dito a respeito de $K_{1}$ e $K_{2}$ ? Para algum desses espaços $X$ é possível concluir que $K_{1} \approx K_{2}$ ?

A dificuldade em se obter uma solução para este problema e também extensões vetoriais para o Teorma de Amir-Cambern 2.2 pode ser resumida na seguinte proposição:

Proposição 3.3. Para cada $\epsilon>0$ existe um espaço de Banach $X$ de cotipo finito tal que para cada compacto métrico enumerável $K$ existe um compacto métrico enumerável $J$ satisfazendo

$$
C(K, X) \stackrel{1+\epsilon}{\sim} C(J, X) \text { e } K \not \approx J .
$$


Demonstração. Fixe $1<p<\infty$ tal que $\sqrt[p]{2}<1+\epsilon$. Seja $l_{p} \oplus l_{p}$ a soma direta de duas cópias de $l_{p}$ com a noma do máximo. Defina o operador $T: l_{p} \rightarrow l_{p} \oplus l_{p}$, para cada $\left(x_{n}\right)_{n \in \mathbb{N}} \in l_{p}$, por

$$
T\left(\left(x_{n}\right)_{n \in \mathbb{N}}\right)=\left(\left(x_{2 n}\right)_{n \in \mathbb{N}},\left(x_{2 n-1}\right)_{n \in \mathbb{N}}\right) .
$$

É fácil verificar que $T$ é um isomorfismo sobrejetor que satisfaz

$$
\|T\|\left\|T^{-1}\right\|=\sqrt[p]{2}<1+\epsilon
$$

Seja $K$ um compacto métrico enumerável. A partir do isomorfismo acima, de maneira natural, é possível construir um isomorfismo de $C\left(K, l_{p}\right)$ em $C\left(K, l_{p} \oplus l_{p}\right)$ com distorção estritamente menor que $1+\epsilon$.

Pelo clássico teorema de Mazurkiewicz e Sierpiński, ver [39] ou [47, Teorema 8.6.10], $K$ é homeomorfo a um intervalo de ordinal $\left[1, \omega^{\alpha} m\right]$ com $0 \leq \alpha<\omega_{1}$ e $1 \leq m<\omega$. Podemos substituir $C\left(K, l_{p}\right)$ por $C\left(\left[1, \omega^{\alpha} m\right], l_{p}\right)$ e $C\left(K, l_{p} \oplus l_{p}\right)$ por $C\left(\left[1, \omega^{\alpha} m\right], l_{p} \oplus l_{p}\right)$ pois estes espaços são isometricamente isomorfos. Dessa forma,

$$
C\left(\left[1, \omega^{\alpha} m\right], l_{p}\right) \stackrel{1+\epsilon}{\sim} C\left(\left[1, \omega^{\alpha} m\right], l_{p} \oplus l_{p}\right) .
$$

Naturalmente vale a relação

$$
C\left(\left[1, \omega^{\alpha} m\right], l_{p} \oplus l_{p}\right)=C\left(\left[1, \omega^{\alpha} m\right], l_{p}\right) \oplus C\left(\left[1, \omega^{\alpha} m\right], l_{p}\right)=C\left(\left[1, \omega^{\alpha} 2 m\right], l_{p}\right) .
$$

Fixe $J=\left[1, \omega^{\alpha} 2 m\right]$. Combinando (3.2) e (3.1) obtemos

$$
C\left(K, l_{p}\right)=C\left(\left[1, \omega^{\alpha} m\right], l_{p}\right) \stackrel{1+\epsilon}{\sim} C\left(\left[1, \omega^{\alpha} 2 m\right], l_{p}\right)=C\left(J, l_{p}\right)
$$

e de acordo com [47, Proposição 8.6.9], $K \not \approx J$.

Contudo, é possível obter respostas para o Problema 3.2. O primeiro resultado nos permite deduzir que para intervalos compactos de ordinais $K_{1}$ e $K_{2}$, uma particular soma topológica de espaços $K_{1}$ é homeomorfa a uma particular soma topológica de espaços $K_{2}$. Mais precisamente, temos o seguinte teorema:

Teorema 3.4. Sejam $X$ um espaço de Banach de cotipo finito, $\alpha$ e $\beta$ ordinais quaisquer $e 1 \leq$ $m, n<\omega$. Então

$$
C\left(\left[1, \omega^{\alpha} m\right], X\right) \stackrel{3}{\sim} C\left(\left[1, \omega^{\beta} n\right], X\right) \Longrightarrow \alpha=\beta
$$

No teorema acima, de acordo com a Proposição 3.3, não podemos concluir que $m=n$ em geral. Também não sabemos se o número 3 pode ser substituido por 4 nem mesmo no caso escalar, onde $X=\mathbb{R}$ ou $X=\mathbb{C}$. Contudo, impondo certa restrição geométrica ao espaço de Banach $X$ podemos obter um novo resultado. Por simplicidade, denotaremos por $X^{n}$ a soma direta de $n$ cópias de um espaço de Banach $X$, munida da norma do máximo.

Teorema 3.5. Seja $X$ um espaço de Banach de cotipo finito tal que $X^{n}$ não contenha cópia de $X^{n+1}$ para todo $n \in \mathbb{N}$. Então, para quaisquer intervalos compactos de ordinais $K_{1}$ e $K_{2}$,

$$
C\left(K_{1}, X\right) \stackrel{3}{\sim} C\left(K_{2}, X\right) \Longrightarrow K_{1} \approx K_{2}
$$

Observe que qualquer espaço vetorial de dimensão finita $X$ não trivial verifica a condição geométrica do Teorema 3.5. Em particular, se $K_{1}$ e $K_{2}$ são intervalos de ordinais enumeráveis e $X=\mathbb{R}$ ou $X=\mathbb{C}$, por [47, Teorema 8.6.10], o Teorema 3.5 coincide com o Teorema 3.1. Este resultado permite, para certos espaços compactos $K_{1}$ e $K_{2}$ e certos espaços de Banach $X$, substituir $1<\lambda \leq 2$ por 3 no Teorema 2.4. 
Os resultados obtidos neste capítulo são baseados nas noções de derivado, compactos dispersos e altura. Estes conceitos serão apresentados na sequência.

Definição 3.6. Seja $K$ um espaço topológico. Para um número ordinal $\alpha$, o $\alpha$-derivado de CantorBendixson de $K, K^{(\alpha)}$ é definido por indução transfinita: $K^{(0)}=K, K^{(1)}$ é o conjunto dos pontos não isolados de $K$ e

$$
K^{(\alpha)}= \begin{cases}\left(K^{(\beta)}\right)^{(1)} & \text { se } \alpha=\beta+1 \\ \bigcap_{\beta<\alpha} K^{(\beta)} & \text { se } \alpha \text { é um ordinal limite. }\end{cases}
$$

Definição 3.7. Um espaço topológico compacto $K$ é chamado de disperso se todo subconjunto fechado $L \subset K$ tem um ponto isolado em $L$.

Observação. Um compacto $K$ é disperso se e somente se $K^{(\alpha)}=\varnothing$ para algum ordinal $\alpha$. De fato, se $K$ não fosse disperso, existiria um conjunto perfeito não vazio $L \subset K$ (i.e., um conjunto fechado tal que todos seus pontos são de acumulação em $L)$. Então $L \subset K^{(\alpha)}$ para todo ordinal $\alpha$ e consequentemente $K^{(\alpha)} \neq \varnothing$. Por outro lado, se $K^{(\alpha)} \neq \varnothing$ para todo $\alpha$, fixe um ordinal $\alpha_{0}$ satisfazendo $K^{\left(\alpha_{0}\right)}=K^{\left(\alpha_{0}+1\right)}$. Claramente $K^{\left(\alpha_{0}\right)}$ é um subconjunto perfeito de $K$ e portanto $K$ não é disperso por definição.

Definição 3.8. Se $K$ for um compacto disperso, o menor ordinal $\alpha$ tal que $K^{(\alpha)}=\varnothing$ será chamado de altura de $K$ e será denotado por ht $(K)$.

As técnicas desenvolvidas neste capítulo permitem também obter uma extensão a valores vetoriais de outro resultado de Gordon em [31]. Um teorema sobre a estabilidade da cardinalidade dos $\alpha$-derivados dos compactos $K_{1}$ e $K_{2}$ via um isomorfismo entre os espaços $C\left(K_{1}, X\right)$ e $C\left(K_{2}, X\right)$ com distorção estritamente menor que 3 .

Teorema 3.9. Sejam $X$ um espaço de Banach de cotipo finito, $K_{1}$ e $K_{2}$ compactos de Hausdorff. Então

$$
C\left(K_{1}, X\right) \stackrel{3}{\sim} C\left(K_{2}, X\right) \Longrightarrow\left|K_{1}^{(\alpha)}\right|=\left|K_{2}^{(\alpha)}\right|
$$

para todo ordinal $\alpha$, diferente de $-1+\mathrm{ht}\left(K_{2}\right)$ no caso em que $K_{2}$ é disperso.

Todos os resultados acima são consequências do estudo sobre isomorfismos de espaços $C\left(K_{1}\right)$ em espaços $C\left(K_{2}, X\right)$ com distorção menor que 3 , onde $K_{1}$ e $K_{2}$ são espaços compactos de Hausdorff e $X$ é um espaço de Banach de cotipo finito. Este estudo será apresentado na Seção 3.2. Na Seção 3.3 demonstraremos os Teoremas 3.4, 3.5 e 3.9.

Na Seção 3.4 apresentaremos uma coleção consistindo de $2^{\omega_{0}}$ espaços de Banach separáveis de dimensão infinita satisfazendo as hipóteses do Teorema 3.5. Em contraste com a Proposição 3.3, cada um desses espaços contém cópia complementada de algum $l_{p}, 1 \leq p<\infty$.

\subsection{Sobre isomorfismos de $C\left(K_{1}\right)$ em espaços $C\left(K_{2}, X\right)$}

Vamos desenvolver nesta seção um resultado fundamental para as demonstrações dos teoremas deste capítulo. Precisamos enriquecer nossa terminologia com mais algumas definições.

Sejam $K_{1}$ e $K_{2}$ espaços topológicos. Denotaremos por $\Lambda: K_{1} \rightrightarrows K_{2}$ uma função definida em $K_{1}$ e assumindo valores nas partes de $K_{2}$, mais precisamente, $\Lambda(x) \subset K_{2}$ para todo $x \in K_{1}$. A possibilidade de que $\Lambda(x)=\varnothing$ para algum $x \in K_{1}$ será admitida. Para um estudo mais detalhado sobre esta classe de funções recomendamos [8].

Definição 3.10. Sejam $K_{1}$ e $K_{2}$ espaços topológicos e uma função $\Lambda: K_{1} \rightrightarrows K_{2}$ como acima. 
(a) O gráfico da função $\Lambda$, denotado por Graf, é o conjunto

$$
\operatorname{Graf}(\Lambda)=\left\{(x, y) \in K_{1} \times K_{2}: y \in \Lambda(x)\right\} .
$$

(b) A imagem da função $\Lambda$, denotada por $R(\Lambda)$, é a projeção de $\operatorname{Graf}(\Lambda)$ sobre a segunda coordenada

$$
R(\Lambda)=\left\{y \in K_{2}: \text { Existe } x \in K_{1} \text { tal que } y \in \Lambda(x)\right\}
$$

(c) A imagem inversa de um conjunto $F \subset K_{2}$, denotada por $\Lambda^{-1}(F)$, é definida por

$$
\Lambda^{-1}(F)=\left\{x \in K_{1}: \Lambda(x) \cap F \neq \varnothing\right\} .
$$

Observação. Naturalmente o gráfico $\operatorname{Graf}(\Lambda)$ caracteriza a função $\Lambda$, i.e., $\operatorname{Graf}\left(\Lambda_{1}\right)=\operatorname{Graf}\left(\Lambda_{2}\right)$ se e somente se $\Lambda_{1}(x)=\Lambda_{2}(x)$ para todo $x$.

Teorema 3.11. Sejam $X$ um espaço de Banach de cotipo finito, $K_{1}$ e $K_{2}$ espaços topológicos compactos de Hausdorff. Suponha existir um isomorfismo $T$ de $C\left(K_{1}\right)$ em $C\left(K_{2}, X\right)$ com $\|T\|\left\|T^{-1}\right\|<3$. Então

(a) Se $K_{2}$ for disperso então $K_{1}$ é disperso e $\mathrm{ht}\left(K_{1}\right) \leq \mathrm{ht}\left(K_{2}\right)$.

(b) Para todo ordinal $\alpha, K_{2}^{(\alpha)}$ é finito ou $\left|K_{1}^{(\alpha)}\right| \leq\left|K_{2}^{(\alpha)}\right|$.

Demonstração. Sem perda de generalidade podemos supor que $\|f\| \leq\|T f\|$ para cada $f \in C_{0}\left(K_{1}\right)$, pois do contrário podemos simplesmente substituir $T$ pelo isomorfismo $\left\|T^{-1}\right\| T$ que claramente possui estas propriedades. Em seguida, fixamos $\epsilon=(1-\|T\| / 3) / 2$ e definimos para cada $x \in K_{1}$ os conjuntos

$$
\begin{aligned}
& \mathcal{F}_{x}=\left\{f \in C\left(K_{1}\right): 0 \leq f \leq 1 \text { e } f(x)>\|T\| / 3\right\}, \\
& \Lambda_{x}=\left\{y \in K_{2}:\|T f(y)\| \geq \epsilon \text { para cada } f \in \mathcal{F}_{x}\right\} .
\end{aligned}
$$

Para demonstrar os itens (a) e (b) do teorema precisamos verificar três afirmações referentes aos conjuntos $\Lambda_{x}$.

Afirmação 1. $\Lambda_{x}$ é um conjunto fechado e não vazio para todo $x \in K_{1}$.

De fato, $\Lambda_{x}$ é a interseção de uma coleção de conjuntos fechados e portanto é fechado. Para demonstrar que é não vazio vamos verificar que esta coleção de fechados tem a propriedade da interseção finita. Sejam $f_{1}, \ldots, f_{n} \in \mathcal{F}_{x}$ e fixe $g=\min _{1 \leq i \leq n} f_{i}$. Então $g \in \mathcal{F}_{x}$ e para cada $1 \leq i \leq n$ vale

$$
\left\|1+2 g-2 f_{i}\right\| \leq 1
$$

Em virtude de

$$
\|T(1+2 g)\| \geq\|1+2 g\|>1+2\|T\| / 3
$$

existe um ponto $y \in K_{2}$ tal que

$$
\|T(1+2 g)(y)\|>1+2\|T\| / 3 .
$$

Mostraremos que $\left\|T f_{i}(y)\right\| \geq \epsilon$ para todo $i$. De fato, se isso fosse falso para algum $1 \leq i \leq n$ então

$$
\begin{aligned}
\|T\| & \geq\left\|T\left(1+2 g-2 f_{i}\right)\right\| \geq\left\|T\left(1+2 g-2 f_{i}\right)(y)\right\| \\
& \geq\|T(1+2 g)(y)\|-2\left\|T f_{i}(y)\right\|>1+2\|T\| / 3-2 \epsilon
\end{aligned}
$$


o que contradiz a escolha de $\epsilon$.

Afirmação 2. Seja $\Lambda: K_{1} \rightrightarrows K_{2}$ definida, para todo $x \in K_{1}$, por $\Lambda(x)=\Lambda_{x}$. Então existe $m \in \mathbb{N}$ tal que $\left|\Lambda^{-1}(y)\right| \leq m$ para todo $y \in K_{2}$.

Sejam $y \in K_{2}$ e $x_{1}, \ldots, x_{n}$ elementos distintos de $\Lambda^{-1}(y)$. Aplicando o Lema de Urysohn 1.12 podemos fixar funções $f_{i} \in \mathcal{F}_{x_{i}}, 1 \leq i \leq n$, com suportes mutuamente disjuntos e satisfazendo

$$
\left\|T f_{i}(y)\right\| \geq \epsilon .
$$

Por hipótese, $X$ tem cotipo finito $2 \leq q<\infty$. Segue da Definição 2.6 que existe uma constante $Q>0$ tal que para quaisquer vetores $v_{1}, v_{2}, \ldots, v_{p}$ em $X$, sempre que $0<\delta \leq\left\|v_{i}\right\|$ para cada $1 \leq i \leq p$, existe uma escolha apropriada de escalares $r_{i}= \pm 1$ verificando

$$
\delta \cdot Q \cdot \sqrt[q]{p} \leq\left\|\sum_{i=1}^{p} r_{i} \cdot v_{i}\right\|
$$

Dessa forma, em virtude de $0<\epsilon \leq\left\|T f_{i}(y)\right\|$ para cada $1 \leq i \leq n$, existe uma escolha apropriada de escalares $r_{i}= \pm 1$ satisfazendo

$$
\epsilon \cdot Q \cdot \sqrt[q]{n} \leq\left\|\sum_{i=1}^{n} r_{i} \cdot T f_{i}(y)\right\|
$$

Como as funções $f_{i}$ têm suportes mutuamente disjuntos, vale $\left\|\sum_{i=1}^{n} r_{i} \cdot f_{i}\right\| \leq 1$. Então

$$
\epsilon \cdot Q \cdot \sqrt[q]{n} \leq\left\|\sum_{i=1}^{n} r_{i} \cdot T f_{i}(y)\right\|=\left\|T\left(\sum_{i=1}^{n} r_{i} \cdot f_{i}\right)(y)\right\| \leq\left\|T\left(\sum_{i=1}^{n} r_{i} \cdot f_{i}\right)\right\| \leq\|T\| \leq 3
$$

e consequentemente $n \leq\left(\frac{3}{\epsilon Q}\right)^{q}$. Podemos concluir que se $m$ é um inteiro positivo tal que $\left(\frac{3}{\epsilon Q}\right)^{q}<$ $m$, então $\left|\Lambda^{-1}(y)\right| \leq m$ para todo $y \in K_{2}$.

Afirmação 3. A imagem $R=R(\Lambda)$ é um subconjunto fechado de $K_{1}$ e $K_{1}^{(\alpha)} \subset \Lambda^{-1}\left(R^{(\alpha)}\right)$ para todo ordinal $\alpha$.

Para verificar essa afirmação considere o gráfico da função $\Lambda$

$$
\operatorname{Graf}(\Lambda)=\left\{(x, y) \in K_{1} \times K_{2}: y \in \Lambda_{x}\right\} .
$$

Seja $\left(\left(x_{i}, y_{i}\right)\right)_{i \in I}$ uma rede em $\operatorname{Graf}(\Lambda)$ convergindo a $(x, y)$. Dado $f \in \mathcal{F}_{x}$ então $f(x)>\|T\| / 3$ e, por continuidade, existe $i_{0} \in I$ tal que $f\left(x_{i}\right)>\|T\| / 3$ para cada $i \geq i_{0}$. Portanto $f \in \mathcal{F}_{x_{i}}$ para todo $i \geq i_{0}$. Em virtude de $y_{i} \in \Lambda_{x_{i}}$ temos $\left\|T f\left(y_{i}\right)\right\| \geq \epsilon$ para cada $i \geq i_{0}$ e por continuidade $\|T f(y)\| \geq \epsilon$. Deduzimos que $(x, y) \in \operatorname{Graf}(\Lambda)$ e isso demonstra que $\operatorname{Graf}(\Lambda)$ é fechado. Em particular o conjunto $R=R(\Lambda)$, que é a projeção de $\operatorname{Graf}(\Lambda)$ sobre a segunda coordenada, também é fechado.

Vamos demonstrar por indução transfinita que $K_{1}^{(\alpha)} \subset \Lambda^{-1}\left(R^{(\alpha)}\right)$, ou seja, que $\Lambda_{x} \cap R^{(\alpha)} \neq \varnothing$ para todo $x \in K_{1}^{(\alpha)}$.

Se $\alpha=0$ então $\Lambda_{x} \subset R$ e, de acordo com a Afirmação $1, \Lambda_{x} \cap R^{(0)}=\Lambda_{x} \neq \varnothing$ para todo $x \in K_{1}$.

Agora, assuma que $\alpha$ é um ordinal limite e fixe $x \in K_{1}^{(\alpha)}$. Da hipótese de indução, para todo $\beta<\alpha$ temos

$$
\Lambda_{x} \cap R^{(\beta)} \neq \varnothing
$$


Estes conjuntos formam uma coleção decrescente de compactos não vazios e portanto tem interseção não vazia. Temos

$$
\Lambda_{x} \cap R^{(\alpha)}=\Lambda_{x} \cap\left(\bigcap_{\beta<\alpha} R^{(\beta)}\right)=\bigcap_{\beta<\alpha}\left(\Lambda_{x} \cap R^{(\beta)}\right) \neq \varnothing .
$$

Em seguida, suponha que $\alpha=\delta+1$. Dado $x \in K_{1}^{(\alpha)}$ existe uma rede $\left(x_{i}\right)_{i \in I}$ no conjunto $K_{1}^{(\delta)} \backslash\{x\}$ convergindo a $x$. Aplicando a hipótese de indução, para cada $i \in I$ podemos fixar $y_{i} \in \Lambda_{x_{i}} \cap R^{(\delta)}$. Em virtude de $R^{(\delta)}$ ser um conjunto compacto existe uma sub-rede, que denotaremos por $\left(y_{j}\right)_{j \in J}$, convergindo a algum $y \in R^{(\delta)}$. Considere também a sub-rede $\left(x_{j}\right)_{j \in J}$ a qual converge para $x$. Dessa forma $\left(\left(x_{j}, y_{j}\right)\right)_{j \in J}$ é uma rede em $\operatorname{Graf}(\Lambda)$ convergindo a $(x, y)$. Concluímos que $y \in \Lambda_{x}$ pois $\operatorname{Graf}(\Lambda)$ é fechado. Observe que para cada $j_{0} \in J$ existe $j \geq j_{0}$ tal que $y_{j} \neq y$, pois do contrário seria possível obter infinitos elementos distintos $\left\{x_{j_{1}}, x_{j_{2}}, \ldots\right\}$ satisfazendo

$$
y \in \bigcap_{1 \leq n} \Lambda_{x_{j_{n}}}
$$

o que contradiz a Afirmação 2. Portanto $y \in R^{(\delta+1)}$.

Agora estamos em condições de demonstrar os itens (a) e (b) de nosso teorema.

(a) Da Afirmação 3 deduzimos que se $K_{2}$ for disperso então $K_{1}$ é disperso e vale a relação

$$
\operatorname{ht}\left(K_{1}\right) \leq \operatorname{ht}(R) \leq \operatorname{ht}\left(K_{2}\right) .
$$

(b) De acordo com a Afirmação 2 existe $m \in \mathbb{N}$ tal que $\left|\Lambda^{-1}(y)\right| \leq m$ para cada $y \in K_{2}$. Se para algum ordinal $\alpha$ o conjunto $K_{2}^{(\alpha)}$ é infinito, então

$$
\left|K_{1}^{(\alpha)}\right| \leq\left|\Lambda^{-1}\left(R^{(\alpha)}\right)\right|=\left|\bigcup_{y \in R^{(\alpha)}} \Lambda^{-1}(y)\right| \leq\left|R^{(\alpha)}\right| \cdot m \leq\left|K_{2}^{(\alpha)}\right| .
$$

\subsection{Demonstrações dos teoremas}

Nosso objetivo nesta seção é demonstrar os Teoremas 3.4, 3.5 e 3.9. Iniciamos com o Teorema 3.4 .

Demonstração do Teorema 3.4. Seja $T$ um isomorfismo de $C\left(\left[1, \omega^{\alpha} m\right], X\right)$ em $C\left(\left[1, \omega^{\beta} n\right], X\right)$ sobrejetor com $\|T\|\left\|T^{-1}\right\|<3$. O espaço $C\left(\left[1, \omega^{\alpha} m\right], X\right)$ contém um subespaço isometricamente isomorfo a $C\left(\left[1, \omega^{\alpha} m\right]\right)$, portanto, a restrição de $T$ a este subespaço é um isomorfismo de $C\left(\left[1, \omega^{\alpha} m\right]\right)$ em $C\left(\left[1, \omega^{\beta} n\right], X\right)$ com distorção estritamente menor que 3. Aplicando o item (a) do Teorema 3.11 deduzimos que

$$
\alpha+1=\operatorname{ht}\left(\left[1, \omega^{\alpha} m\right]\right) \leq \operatorname{ht}\left(\left[1, \omega^{\beta} n\right]\right)=\beta+1,
$$

$\operatorname{logo}, \alpha \leq \beta$. Em virtude de $T$ ser um isomorfismo sobrejetor, de maneira análoga verifica-se que $\beta \leq \alpha$.

Em seguida, demonstraremos o Teorema 3.9. 
Demonstração do Teorema 3.9. Seja $T$ um isomorfismo sobrejetor de $C\left(K_{1}, X\right)$ em $C\left(K_{2}, X\right)$ com distorção $\|T\|\left\|T^{-1}\right\|<3$. Distinguimos dois casos:

Caso 1: $K_{2}$ é disperso. O espaço $C\left(K_{1}, X\right)$ contém um subespaço isometricamente isomorfo a $C\left(K_{1}\right)$, portanto, a restrição de $T$ a este subespaço é um isomorfismo de $C\left(K_{1}\right)$ em $C\left(K_{2}, X\right)$ satisfazendo as hipóteses do Teorema 3.11. Aplicando o item (a) do Teorema 3.11 deduzimos que $K_{1}$ é disperso e ht $\left(K_{1}\right) \leq \mathrm{ht}\left(K_{2}\right)$. Mais ainda, restringindo $T^{-1}$ a $C\left(K_{2}\right)$ e aplicando novamente o item (a) do Teorema 3.11 podemos concluir que ht $\left(K_{2}\right) \leq \mathrm{ht}\left(K_{1}\right)$. Logo ht $\left(K_{2}\right)=\mathrm{ht}\left(K_{1}\right)$.

Se $\alpha>-1+$ ht $\left(K_{2}\right)$, então os conjuntos $K_{1}^{(\alpha)}$ e $K_{2}^{(\alpha)}$ são ambos vazios e portanto têm a mesma cardinalidade. Por outro lado, se $\alpha<-1+\mathrm{ht}\left(K_{2}\right)$, então $K_{1}^{(\alpha)}$ e $K_{2}^{(\alpha)}$ são ambos infinitos. Aplicando o item (b) do Teorema 3.11 duas vezes obtemos $\left|K_{1}^{(\alpha)}\right|=\left|K_{2}^{(\alpha)}\right|$.

Caso 2: $K_{2}$ não é disperso. Procendendo como no Caso 1 concluímos que $K_{1}$ também não é disperso. Então, para todo ordinal $\alpha$ os conjuntos $K_{1}^{(\alpha)}$ e $K_{2}^{(\alpha)}$ são ambos infinitos. Aplicando o item (b) do Teorema 3.11 duas vezes obtemos $\left|K_{1}^{(\alpha)}\right|=\left|K_{2}^{(\alpha)}\right|$. Isto completa a demonstração do Teorema 3.9.

Recordamos que um conjunto fechado não vazio $A \subset K$ admite um operador de extensão simultânea regular se existe um operador linear contínuo $L: C(A) \rightarrow C(K)$ satisfazendo $\|L\|=1$, $L\left(\mathbb{1}_{A}\right)=\mathbb{1}_{K} \mathrm{e}$

$$
\left.L f\right|_{A}=f, f \in C(A) .
$$

É bem conhecido que tais extensões existem sempre que $K$ é metrizável [47, Teorema 21.1.4]. No caso em que $K$ for o intervalo de ordinal $[1, \alpha]$, podemos construir um operador de extensão simultânea regular $L: C(A) \rightarrow C(K)$ do seguinte modo. Para cada $f \in C(A)$ definimos, para cada $\beta \leq \alpha$, $L f(\beta)=f(\gamma)$ onde

$$
\gamma= \begin{cases}\inf \{[\beta, \alpha] \cap A\} & \text { se }[\beta, \alpha] \cap A \neq \varnothing \\ \sup A & \text { se }[\beta, \alpha] \cap A=\varnothing .\end{cases}
$$

Demonstra-se, sem dificuldades, que $L$ é um operador de extensão simultânea regular de $C(A)$ em $C([1, \alpha])$, ver [2, Proposição 1.1].

Proposição 3.12. Sejam $X$ um espaço de Banach de cotipo finito, $K_{1}$ e $K_{2}$ espaços compactos de Hausdorff, $T: C\left(K_{1}, X\right) \rightarrow C\left(K_{2}, X\right)$ um isomorfismo satisfazendo $\|f\| \leq\|T f\|$ para todo $f \in C\left(K_{1}, X\right)$ e $\|T\|<3, \epsilon=(1-\|T\| / 3) / 2$ e $A \subset K_{1}$ um subconjunto fechado admitindo um operador de extensão simultânea regular. Para todo ordinal $\alpha$, se $A^{(\alpha)} \neq \varnothing$, então para cada $\gamma \in C\left(K_{1}, X\right)$ verificando $\|\gamma\|=1 e\|\gamma(x)\|=1$ para todo $x \in A$, existe $y \in K_{2}^{(\alpha)}$ tal que $\|T \gamma(y)\| \geq \epsilon$.

Demonstração. Fixe $\gamma \in C\left(K_{1}, X\right)$ tal que $\|\gamma\|=1$ e $\|\gamma(x)\|=1$ para todo $x \in A$. Seja $L: C(A) \rightarrow$ $C\left(K_{1}\right)$ um operador de extensão simultânea regular e considere o operador $T^{A}: C(A) \rightarrow C\left(K_{2}, X\right)$ definido por

$$
T^{A} f=T(\gamma \cdot L f)
$$

Então $\left\|T^{A}\right\|<3$ e $\|f\| \leq\left\|T^{A} f\right\|$ para todo $f \in C(A)$. De fato, para cada $x \in A$

$$
\left\|T^{A} f\right\|=\|T(\gamma \cdot L f)\| \geq\|\gamma \cdot L f\| \geq\|\gamma(x) \cdot f(x)\|=|f(x)| .
$$

Fixe $\epsilon_{A}=\left(1-\left\|T^{A}\right\| / 3\right) / 2$. Nessas condições, como na prova do Teorema 3.11, para todo $x \in A$ podemos definir os conjuntos $\mathcal{F}_{x}^{A}, \Lambda_{x}^{A}$ e uma função $\Lambda^{A}: A \rightrightarrows K_{2} \operatorname{com}$ imagem $R_{A}=R\left(\Lambda^{A}\right) \subseteq K_{2}$ 
associados ao operador $T^{A}$. De acordo com a Afirmação 3 no referido teorema, para todo número ordinal $\alpha$ temos

$$
A^{(\alpha)} \subset\left(\Lambda^{A}\right)^{-1}\left(R_{A}^{(\alpha)}\right)
$$

Em particular, se $A^{(\alpha)} \neq \varnothing$, então existe um $y \in R_{A}^{(\alpha)} \subset K_{2}^{(\alpha)}$ tal que

$$
\|T \gamma(y)\|=\left\|T^{A_{1}} \mathbb{1}_{A}(y)\right\| \geq \epsilon_{A} \geq \epsilon .
$$

Estamos agora em condições de demonstrar o Teorema 3.5.

Demonstração do Teorema 3.5. Seja $T$ um isomorfismo sobrejetor de $C\left(K_{1}, X\right)$ em $C\left(K_{2}, X\right)$ com $\|T\|\left\|T^{-1}\right\|<3$. Se $K_{1}$ e $K_{2}$ são intervalos compactos de ordinais, por [47, Teorema 8.6.5], existem ordinais $\alpha, \beta$ e $1 \leq n, m<\omega$ tais que

$$
K_{1} \approx\left[1, \omega^{\alpha} n\right] \text { e } K_{2} \approx\left[1, \omega^{\beta} m\right] .
$$

Aplicando o Teorema 3.4 obtemos $\alpha=\beta$. Precisamos verificar que $m=n$. Substituindo o operador $T$ por $\left\|T^{-1}\right\| T$ se necessário, vamos supor que $\|f\| \leq\|T f\|$ para cada $f \in C\left(K_{1}, X\right)$.

Em virtude de $K_{1}$ ser um compacto de Hausdorff disperso, $K_{1}$ é totalmente desconexo e portanto é zero-dimensional, i. e., possui uma base constituída apenas de conjuntos aberto-fechados, ver [47, Proposição 8.2.2].

Escreva $K_{1}^{(\alpha)}=\left\{x_{1}, \ldots, x_{n}\right\}$. Por $K_{1}$ ser de Hausdorff, os pontos $x_{1}, \ldots, x_{n}$ podem ser separados por abertos mutuamente disjuntos; por $K_{1}$ ser zero-dimensional, podemos diminuir estes conjuntos abertos para conjuntos abertos-fechados. Obtemos dessa forma conjuntos abertos-fechados $U_{1}, \ldots, U_{n}$ mutuamente disjuntos satisfazendo $x_{j} \in U_{j}$ para todo $1 \leq j \leq n$. Para cada $1 \leq j \leq n-1$ defina $A_{j}=U_{j}$ e $A_{n}$ como o conjunto complementar da reunião de $U_{1}, U_{2}, \ldots, U_{n-1}$. Assim, os conjuntos $A_{1}, \ldots, A_{n}$ são abertos-fechados mutuamente disjuntos tais que $x_{j} \in A_{j} \mathrm{e}$

$$
K_{1}=\bigcup_{j=1}^{n} A_{j}
$$

Em seguida, identificamos $X^{n}$ e $X^{m} \operatorname{com} C\left(K_{1}^{(\alpha)}, X\right)$ e $C\left(K_{2}^{(\alpha)}, X\right)$ respectivamente. Associamos a cada $z=\left(z_{1}, \ldots, z_{n}\right) \in X^{n}$ a função

$$
\gamma_{z}=\sum_{j=1}^{n} z_{j} \cdot \mathbb{1}_{A_{j}} \in C\left(K_{1}, X\right) .
$$

Observe que $\left\|\gamma_{z}\right\|=\|z\|, z \in X^{n}$. Considere a aplicação $S: X^{n} \rightarrow X^{m}$ definida por

$$
S(z)=\left.T \gamma_{z}\right|_{K_{2}^{(\alpha)}}, z \in X^{n}
$$

Claramente $S$ é um operador linear e verifica $\|S\| \leq\|T\|$. Seja $z=\left(z_{1}, \ldots, z_{n}\right) \in B_{X^{n}}$ e, sem perda de generalidade, suponha que $\|z\|=\left\|z_{1}\right\|=1$.

Fixe $\epsilon=(1-\|T\| / 3) / 2$. Aplicando a Proposição $3.12 \operatorname{com} A=A_{1}$ e $\gamma=\gamma_{z}$, concluímos que existe um $y \in K_{2}^{(\alpha)}$ tal que

$$
\|S z\| \geq\|S z(y)\|=\left\|T \gamma_{z}(y)\right\| \geq \epsilon .
$$

Deduzimos que para todo $z \in X^{n}$ vale

$$
\epsilon\|z\| \leq\|S z\| \leq\|T\|\|z\| .
$$


Por hipótese, $X^{m}$ não contém subespaço isomorfo a $X^{m+1}$ e isso nos permite concluir que $n \leq m$. Demonstra-se de maneira análoga que $m \leq n$.

\subsection{Considerações finais}

Apresentaremos nesta seção uma coleção de espaços de Banach $X$ satisfazendo as condições do Teorema 3.5, i.e, $X$ tem cotipo finito e $X^{n}$ não contém subespaço isomorfo a $X^{n+1}$ para todo $n \in \mathbb{N}$. Claramente, qualquer espaço de dimensão finita não trivial possui essa propriedade e nosso objetivo é obter exemplos de dimensão infinita. Para exibir tais exemplos devemos recordar algumas definições e alguns teoremas. As definições apresentadas nesta seção pode ser encontradas em [1], [27] e [28].

Para espaços de Banach $X$ e $Y$, denotamos por $\mathcal{L}(X, Y)$ o espaço de todos os operadores lineares contínuos de $X$ em $Y$.

Definição 3.13. Sejam $X$ e $Y$ espaços de Banach. Um operador $T \in \mathcal{L}(X, Y)$ é denominado

(a) Fredholm: se seu núcleo tem dimensão finita e sua imagem tem codimensão finita

(b) Inessencial: se para todo $S \in \mathcal{L}(Y, X)$ o operador $I_{X}-S \circ T$ for Fredholm, onde $I_{X}$ denota o operador identidade em $X$.

(c) Estritamente Singular: se não existir supespaço fechado $Z$ de $X$, de dimensão infinita, tal que a restrição de $T$ a $Z$ seja um isomorfismo sobre sua imagem.

(d) Improjetivo: se não existir supespaço fechado $Z$ de $X$, de dimensão infinita, tal que a restrição de $T$ a $Z$ seja um isomorfismo sobre sua imagem e $T(Z)$ seja um subespaço complementado de $Y$.

Definição 3.14. Dois espaços de Banach $X$ e $Y$ são essencialmente incomparáveis se todo operador $T \in \mathcal{L}(X, Y)$ for inessencial.

Definição 3.15. Um espaço de Banach $X$ de dimensão infinita é hereditariamente indecomponível (ou H.I.) se nenhum subespaço de $X$ pode ser escrito como direta de dois subespaços fechados de dimensão infinita.

Vamos precisar de alguns teoremas que apresentaremos na sequência.

Teorema 3.16 (Aiena-González). Sejam $X$ e $Y$ espaços de Banach. Se $X$ é hereditariamente indecomponivel, para todo $T \in \mathcal{L}(X, Y), T$ é improjetivo se e somente se $T$ é inessencial.

Demonstração. Ver P. Aiena e M. González [1, Teorema 4.11].

Teorema 3.17 (Galego). Se $\left(X_{1}, Y_{1}\right)$ e $\left(X_{2}, Y_{2}\right)$ são pares de espaços de Banach essencialmente incomparáveis verificando $X_{1} \oplus Y_{2} \sim X_{2} \oplus Y_{1}$, então existem $m, n \in \mathbb{N}$ tais que

$$
X_{1} \oplus \mathbb{K}^{m} \sim X_{2} \oplus \mathbb{K}^{n} .
$$

Demonstração. Ver E. M. Galego [29, Observação 3.3].

Teorema 3.18. Seja $1 \leq p<\infty$. Todo subespaço de dimensão infinita de $l_{p}$ contém um subespaço que é isomorfo a $l_{p}$. 
Demonstração. Ver J. Lindenstrauss e L. Tzafriri [36, Proposição 2.a.2, p. 53].

Teorema 3.19. Seja $1 \leq p<\infty$. Todo subespaço de dimensão infinita e complementado de $l_{p}$ é isomorfo a $l_{p}$.

Demonstração. Ver J. Lindenstrauss e L. Tzafriri [36, Teorema 2.a.3, p. 54]

Teorema 3.20. Seja $T \in \mathcal{L}(X, Y)$ um operador com imagem fechada e tal que $\operatorname{dim} \operatorname{ker} T<\infty$. Seja $S \in \mathcal{L}(X, Y)$ um operador estritamente singular. Então $T+S$ tem imagem fechada e $\operatorname{dim} \operatorname{ker} T+S<$ $\infty$.

Demonstração. Ver J. Lindenstrauss e L. Tzafriri [36, Teorema 2.c.10, p. 79].

Dados espaços de Banach $X$ e $Y$, utilizaremos a notação $Y \hookrightarrow X$ para dizer que $Y$ é isomorfo a algum subespaço de $X$.

Teorema 3.21 (Ferenczi). Seja $n \in \mathbb{N}$. Para cada $i=1, \ldots, n$ seja $X_{i}$ um espaço de Banach hereditariamente indecomponível. Então não existem espaços de Banach de dimensão infinita $Y_{1}, \ldots, Y_{n+1}$ tais que

$$
Y_{1} \oplus \ldots \oplus Y_{n+1} \hookrightarrow X_{1} \oplus \ldots \oplus X_{n} .
$$

Demonstração. Ver V. Ferenczi [28, Corolário 2].

Teorema 3.22 (Samuel). Sejam $X$ e $Y$ espaços de Banach $e 1 \leq p<\infty$. Então $X$ ou $Y$ contém um subespaço isomorfo a $l_{p}$ se e somente se $X \oplus Y$ contém um subespaço isomorfo a $l_{p}$.

Demonstração. Ver C. Samuel [46, Teorema 1].

Denote por $H$ o espaço de Banach separável uniformemente convexo e hereditariamente indecomponível introduzido por Ferenczi em [27] e para cada $1 \leq p<\infty$ defina

$$
H_{p}=l_{p} \oplus H .
$$

Temos o seguinte resultado:

Proposição 3.23. Para cada $1 \leq p<\infty$ e para cada $n \in \mathbb{N}, H_{p}^{n}$ não contém subespaço isomorfo a $H_{p}^{n+1}$. Mais ainda, se $1 \leq p<q<\infty$ então

$$
H_{p} \nsim H_{q} .
$$

Demonstração. De início, observe que todo operador $S \in \mathcal{L}\left(H, l_{r}\right), 1 \leq r<\infty$, é improjetivo. De fato, suponha um subespaço de dimensão infinita $Z$ em $H$ tal que a restrição a $Z$ seja um isomorfismo e $S(Z)$ seja complementado em $l_{r}$. De acordo com Teorema 3.19 temos $Z \sim l_{r}$, o que é um absurdo pois $H$ é hereditariamente indecomponível.

Também pelo fato de $H$ ser hereditariamente indecomponível, segue do Teorma 3.16 que todo operador linear improjetivo $S \in \mathcal{L}\left(H, l_{r}\right)$ é inessencial. Logo, todo operador $S \in \mathcal{L}\left(H, l_{r}\right)$ é inessencial.

Sejam $1 \leq p, q<\infty$. Pelo argumento acima, $\left(l_{p}, H\right)$ e $\left(l_{q}, H\right)$ são pares de espaços de Banach essencialmente incomparáveis. Assim, se $H_{p}=l_{p} \oplus H \sim l_{q} \oplus H=H_{q}$, pelo Teorema 3.17, existem $m, n \in \mathbb{N}$ tais que

$$
l_{p} \oplus \mathbb{K}^{m} \sim l_{q} \oplus \mathbb{K}^{n}
$$


Deduzimos que $p=q$.

Em seguida, dado $1 \leq p<\infty$, assuma que $H_{p}^{n}$ contenha um subespaço isomorfo a $H_{p}^{n+1}$ para algum $n \in \mathbb{N}$. Nessas condições existe um isomorfismo $T: H^{n+1} \rightarrow l_{p} \oplus H^{n}$. Seja $P$ a projeção natural de $l_{p} \oplus H^{n}$ em $l_{p}$.

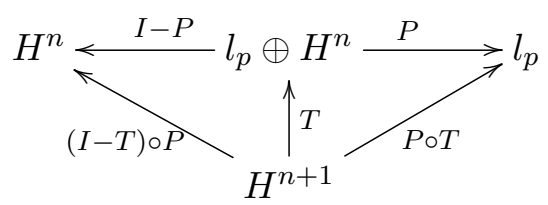

Vamos demonstrar que o operador $P \circ T$ é estritamente singular. Com efeito, suponha por absurdo que exista em $H^{n+1}$ um subespaço de dimensão infinita $Y$ tal que a restrição de $P \circ T$ a $Y$ seja um isomorfismo. Então

$$
Y \hookrightarrow l_{p}
$$

De acordo com o Teorema 3.18,

$$
l_{p} \hookrightarrow Y \hookrightarrow H^{n+1}
$$

e pelo Teorema 3.22

$$
l_{p} \hookrightarrow H,
$$

o que contradiz a hipótese de $H$ ser hereditariamente indecomponível.

Então $P \circ T$ é estritamente singular. Deduzimos, pelo Teorema 3.20, que o operador $(I-P) \circ T$ : $H^{n+1} \rightarrow H^{n}$ tem imagem fechada e seu núcleo é um espaço vetorial de dimensão finita $V$. Seja $W$ um espaço de Banach tal que $H^{n+1}=W \oplus V$. A restrição de $(I-P) \circ T$ a $W$ é um isomorfismo sobre sua imagem, portanto, $H^{n}$ contém um subespaço isomorfo a $W$. Simbolicamente

$$
W \hookrightarrow H^{n} .
$$

Seja $Z$ um subespaço de $H$, tal que $H=V \oplus Z$. Temos

$$
H^{n+1} \oplus Z \sim W \oplus V \oplus Z \hookrightarrow H^{n} \oplus V \oplus Z \sim H^{n+1},
$$

o que, de acordo com o Teorema 3.21, é impossível. 


\section{Capítulo 4}

\section{Sobre distâncias de Banach-Mazur entre espaços $C_{0}(K, X)$ e $C_{0}(\Gamma, X) \operatorname{com} \Gamma$ discreto}

\subsection{Introdução}

Neste capítulo, nosso principal interesse é estudar a distância de Banach-Mazur entre os espaços $C_{0}(K, X)$, onde $K$ denota um espaço topológico localmente compacto de Hausdorff, e os espaços $C_{0}(\Gamma, X)$, onde $\Gamma$ denota um conjunto infinito munido da topologia discreta. Recordamos que para espaços de Banach isomorfos $X$ e $Y$, a distância de Banach-Mazur entre estes espaços é definida por

$$
\mathrm{d}(X, Y)=\inf _{T}\left\{\|T\|\left\|T^{-1}\right\|\right\}
$$

onde $T$ percorre todos os isomorfismos sobrejetores de $X$ em $Y$.

A fonte de nossa pesquisa remonta a Banach. Em 1932, ele afirmou que d $\left(c, c_{0}\right) \leq 4$; ver $[4$, p. 181]. Banach chegou a essa conclusão utilizando o seguinte isomorfismo $T_{\lambda}$ de $c$ em $c_{0}$ :

$$
T_{\lambda}\left(x_{1}, x_{2}, x_{3}, \ldots\right)=\left(\lambda x, x_{1}-x, x_{2}-x, x_{3}-x, \ldots\right),
$$

com $\lambda=1$ e $\left(x_{n}\right)_{n \in \mathbb{N}}$ converge a $x$. Uma melhor estimativa para essa distância pode ser obtida de (4.1) fixando-se $\lambda=2$ a saber: $\mathrm{d}\left(c, c_{0}\right) \leq 3$. Finalmente, em 1968 Cambern [14], veja também [10], calculou o valor exato desta distância:

$$
\mathrm{d}\left(c, c_{0}\right)=3 .
$$

Surge a questão sobre os valores exatos das distâncias de Banach-Mazur entre os espaços $C(K)$, onde $K$ é um compacto de Hausdorff, e o espaço $c_{0}$. Pelo clássico teorema de Mazurkiewicz e Sierpiński [47, Teorema 8.6.10] e por um teorema de Bessaga e Pełczyński [7, Teorema 1] deduzimos que se um espaço $C(K)$ é isomorfo a $c_{0}$, então $K$ é homeomorfo a um intervalo de ordinal $\left[1, \omega^{n} k\right]$, $1 \leq n, k<\omega$. De maneira natural, temos a seguinte pergunta:

Problema 4.1. Quais são os valores de $\mathrm{d}\left(C\left(\left[1, \omega^{n} k\right]\right), c_{0}\right), 1 \leq n, k<\omega$ ?

O objetivo do presente capítulo é duplo. Em primeiro lugar, fornecer uma extensão vetorial de (4.2). Em segundo lugar, resolver completamente o Problema 4.1.

Os principais resultados deste capítulo envolvem a noção de derivado, ver Definição 3.6. O primeiro resultado fornece cotas inferiores para distâncias de Banach-Mazur entre certos espaços $C_{0}(K, X)$. Trata-se de uma generalização do principal resultado de [14], o caso onde $n=1$ e $X=\mathbb{R}$ ou $X=\mathbb{C}$. 
Teorema 4.2. Sejam $1 \leq n<\omega, \Gamma$ um conjunto infinito munido da topologia discreta, $K$ um espaço topológico localmente compacto de Hausdorff e $X$ um espaço de Banach de cotipo finito. Então

$$
C_{0}(K, X) \sim C_{0}(\Gamma, X) \text { e } K^{(n)} \neq \varnothing \Longrightarrow \mathrm{d}\left(C_{0}(K, X), C_{0}(\Gamma, X)\right) \geq 2 n+1 .
$$

Para se obter cotas superiores para distâncias mencionadas no Problema 4.1 demonstramos:

Teorema 4.3. Sejam $1 \leq n, k<\omega$ e $X$ um espaço de Banach. Então

$$
\mathrm{d}\left(C\left(\left[1, \omega^{n} k\right], X\right), C_{0}(\mathbb{N}, X)\right) \leq 2 n+1 .
$$

Então, como consequência imediata dos Teoremas 4.2 e 4.3 obtemos a seguinte generalização de (4.2) que ao mesmo tempo resolve o Problema 4.1.

Corolário 4.4. Sejam $1 \leq n, k<\omega$ e X um espaço de Banach de cotipo finito. Então

$$
\mathrm{d}\left(C\left(\left[1, \omega^{n} k\right], X\right), C_{0}(\mathbb{N}, X)\right)=2 n+1 .
$$

Não sabemos responder quando as asserções do Corolário 4.4 permanecem verdadeiras sem a hipótese de $X$ ser um espaço de Banach de cotipo finito.

O Teorema 4.2 pode ser aplicado para se obter algumas generalizações de resultados clássicos sobre espaços $C_{0}(\Gamma)$. Por exemplo, é bem conhecido que se um espaço $C(K)$ é isomorfo a um espaço $C_{0}(\Gamma)$, onde $\Gamma$ é um conjunto infinito munido da topologia discreta, então $K^{(\omega)}=\varnothing$. Tal resultado pode ser obtido por uma combinação de teoremas de [5], [7] e [43], veja também [37, Teorema 1.1]. Todavia, como consequência imediata do Teorema 4.2, temos a seguinte extensão vetorial deste resultado.

Corolário 4.5. Sejam $\Gamma$ um conjunto discreto infinito, $K$ um espaço localmente compacto de Hausdorff e $X$ um espaço de Banach de cotipo finito. Então

$$
C_{0}(K, X) \sim C_{0}(\Gamma, X) \Longrightarrow K^{(\omega)}=\varnothing .
$$

Demonstração. Seja $T$ um isomorfismo sobrejetor de $C_{0}(K, X)$ em $C_{0}(\Gamma, X)$. Seja $n \in \mathbb{N}$ tal que $\|T\|\left\|T^{-1}\right\|<2 n+1$. Então, pelo Teorema $4.2, K^{(n)}=\varnothing$.

Utilizando o principal resultado do Capítulo 2, mais precisamente o Teorema 2.8, e o Corolário 4.5, para um espaço de Banach $X$ de cotipo finito, separável ou tal que $X^{*}$ tem a propriedade de Radon-Nikodým, temos um classificação de todos os compactos de Hausdorff $K$ tais que $C(K, X) \sim$ $C_{0}(\mathbb{N}, X)$.

Corolário 4.6. Sejam $X$ um espaço de Banach de cotipo finito e $K$ um compacto de Hausdorff. Se $X$ é separável ou $X^{*}$ tem a propriedade de Radon-Nikodým, então

$$
C(K, X) \sim C_{0}(\mathbb{N}, X) \Longrightarrow K \approx\left[1, \omega^{n} k\right], \operatorname{com} 1 \leq k, n<\omega .
$$

Demonstração. De acordo com o Teorema 2.8, se $C(K, X) \sim C_{0}(\mathbb{N}, X)$, então $K$ é enumerável. De acordo com [47, Teorema 8.6.10], o compacto $K$ é homeomorfo a um intervalo de ordinal enumerável. De acordo com o Corolário 4.5 temos $K^{(\omega)}=\varnothing$. Consequentemente, $K \approx\left[1, \omega^{n} k\right]$ onde $1 \leq k, n<$ $\omega$. 
Finalmente, o clássico teorema de Milyutin [44, Teorema 21.5.10] mostra que a hipótese de cotipo finito em geral não pode ser removida no Corolário 4.5. De fato,

$$
\begin{aligned}
C_{0}(\mathbb{N}, C([0,1])) & \sim C([1, \omega], C([0,1])) \sim C([1, \omega] \times[0,1]) \\
& \sim C([0,1]) \sim C([0,1] \times[0,1]) \sim C([0,1], C[0,1]),
\end{aligned}
$$

entretanto, $[0,1]^{(\omega)}=[0,1]$.

\subsection{Resultados preliminares}

Vamos estabelecer nesta seção duas proposições que terão um importante papel na Seção 4.3. Para um espaço de Banach $X$ denotaremos por $S_{X}$ a esfera unitária de $X$. Para um subconjunto $J$ de um espaço topológico $K$ denotaremos por $\stackrel{\circ}{J}$ o conjunto dos pontos interiores de $J$.

Proposição 4.7. Sejam $K$ um espaço localmente compacto de Hausdorff tal que $K^{(n)} \neq \varnothing$ para algum $1 \leq n<\omega, \Gamma$ um conjunto infinito munido da topologia discreta e $X$ um espaço de Banach de cotipo finito. Fixe $v \in S_{X}$ e $0<\epsilon<1$. Se $T$ é um isomorfismo de $C_{0}(K, X)$ em $C_{0}(\Gamma, X)$ com $\left\|T^{-1}\right\|=1$ então existem pontos $x_{1}, \ldots, x_{n} \in K$, subconjuntos compactos $J_{1}, \ldots, J_{n}$ de $K$ e funções $h_{1}, \ldots, h_{n}$ em $C_{0}(K)$ satisfazendo:

(a) $x_{i} \in \stackrel{\circ}{J}_{i} \cap K^{(n-i+1)}$, para $1 \leq i \leq n$.

(b) $J_{i} \subset \stackrel{\circ}{i-1}_{i}$ para $1<i \leq n$.

(c) $0 \leq h_{i} \leq 1, h_{i}(x)=1$ se $x \in J_{i}$, para $1 \leq i \leq n$, e $h_{i}(x)=0$ se $x \notin \stackrel{\circ}{J}_{i-1}$ para $1<i \leq n$.

(d) Os conjuntos $G_{i}=\left\{y \in \Gamma:\left\|T\left(v \cdot h_{i}\right)(y)\right\| \geq \epsilon\right\}, 1 \leq i \leq n$, são não vazios e mutuamente disjuntos.

Demonstração. A demonstração será feita por indução em $n$. Seja $x_{1} \in K^{(n)}$ e fixe $J_{1}$ uma vizinhança compacta de $x_{1}$. Utilizando o Lema de Urysohn 1.12 podemos encontrar $h_{1} \in C_{0}(K)$ com $0 \leq h_{1} \leq 1, h_{1}(x)=1$ se $x \in J_{1}$. Mais ainda, em virtude de $0<\epsilon<1$ e $\left\|T^{-1}\right\|=1$, o conjunto $G_{1}=\left\{y \in \Gamma:\left\|T\left(v \cdot h_{1}\right)(y)\right\| \geq \epsilon\right\}$ é não vazio.

Dado $1<r<n$, suponha que foram obtidos pontos $x_{1}, \ldots, x_{r}$, conjuntos compactos $J_{1}, \ldots, J_{r}$ e funções $h_{1}, \ldots, h_{r}$ em $C_{0}(K)$ verificando os itens $(a),(b),(c)$ e $(d)$.

Por $K$ ser um espaço localmente compacto de Hausdorff, é possível encontrar pontos $a_{1}, a_{2}, a_{3} \ldots$ em $\left(\stackrel{\circ}{J}_{r} \backslash\left\{x_{r}\right\}\right) \cap K^{(n-r)}$, subconjuntos abertos mutuamente disjuntos $U_{1}, U_{2}, U_{3} \ldots$ e compactos $L_{1}, L_{2}, L_{3} \ldots$ verificando

$$
a_{i} \in \stackrel{\circ}{L}_{i} \subset L_{i} \subset U_{i} \subset J_{r}, i \in \mathbb{N} .
$$

Aplicando o Lema de Urysohn 1.12 podemos obter funções $f_{1}, f_{2}, f_{3} \ldots$ em $C_{0}(K)$ tais que, para cada $i \in \mathbb{N}, 0 \leq f_{i} \leq 1, f_{i}(x)=1$ se $x \in L_{i}$ e $f_{i}(x)=0$ se $x \notin U_{i}$. Em virtude de $U_{i} \cap U_{j}=\varnothing$ se $i \neq j$, temos $f_{i} \cdot f_{j}=0$ se $i \neq j$.

Seja $G=G_{1} \cup \ldots \cup G_{r}$. Afirmamos que existe $m \in \mathbb{N}$ tal que

$$
\left\{y \in \Gamma:\left\|T\left(v \cdot f_{m}\right)(y)\right\| \geq \epsilon\right\} \cap G=\varnothing .
$$

Do contrário, supondo $G=\left\{y_{1}, \ldots, y_{s}\right\}$ e denotando

$$
\Lambda_{i}=\left\{j \in \mathbb{N}:\left\|T\left(v \cdot f_{j}\right)\left(y_{i}\right)\right\| \geq \epsilon\right\}, 1 \leq i \leq s,
$$

temos

$$
\mathbb{N} \subseteq \Lambda_{1} \cup \ldots \cup \Lambda_{s}
$$


e portanto $\Lambda_{l}$ é infinito para algum $1 \leq l \leq s$. Sejam $l_{1}, l_{2}, l_{3} \ldots$ inteiros distintos em $\Lambda_{l}$.

Por hipótese, $X$ tem cotipo finito $2 \leq q<\infty$. Segue da Definição 2.6 que existe uma constante $Q>0$ tal que para quaisquer vetores $v_{1}, v_{2}, \ldots, v_{p}$ em $X$, se $0<\eta \leq\left\|v_{i}\right\|$ para cada $1 \leq i \leq p$, existe uma escolha apropriada de escalares $r_{i}= \pm 1$ verificando

$$
\left\|\sum_{i=1}^{p} r_{i} \cdot v_{i}\right\| \geq \eta \cdot Q \cdot \sqrt[q]{p}
$$

Seja $m \in \mathbb{N}$ tal que $\epsilon \cdot Q \cdot \sqrt[q]{m}>\|T\|$. De acordo com (4.4), existe uma escolha apropriada de escalares $r_{i}= \pm 1$ satisfazendo

$$
\left\|\sum_{i=1}^{m} r_{i} \cdot T\left(v \cdot f_{l_{i}}\right)\left(y_{l}\right)\right\| \geq \epsilon \cdot Q \cdot \sqrt[q]{m}>\|T\| .
$$

Em virtude de $f_{l_{i}} \cdot f_{l_{j}}=0$ se $i \neq j$ e $\left\|f_{l_{i}}\right\| \leq 1$ para todo $i$, a função $A=\sum_{i=1}^{m} r_{i} \cdot v \cdot f_{l_{i}} \in C_{0}(K, X)$ verifica $\|A\| \leq 1$. Entretanto,

$$
\|T\| \geq\|T(A)\| \geq\left\|T\left(\sum_{i=1}^{m} r_{i} \cdot v \cdot f_{l_{i}}\right)\left(y_{l}\right)\right\|=\left\|\sum_{i=1}^{m} r_{i} \cdot T\left(v \cdot f_{l_{i}}\right)\left(y_{l}\right)\right\|>\|T\|,
$$

uma contradição que estabelece nossa afirmação.

Portanto, podemos fixar $m \in \mathbb{N}$ verificando (4.3) e definir $x_{r+1}=a_{m}, J_{r+1}=L_{m}, h_{r+1}=f_{m} \mathrm{e}$ $G_{r+1}=\left\{y \in \Gamma:\left\|T\left(v \cdot f_{m}\right)(y)\right\| \geq \epsilon\right\}$. É simples verificar que as condições (a), (b), (c) e (d) estão satisfeitas para $r+1$. Isso completa a demonstração.

Na proposição seguinte utilizaremos o Teorema 1.17 (Representação de Riesz-Singer) e outros resultados do Capítulo 1.

Proposição 4.8. Sejam $X$ um espaço de Banach de cotipo finito, $K$ um espaço localmente compacto de Hausdorff, $\Gamma$ um conjunto infinito munido da topologia discreta e $T$ um isomorfismo de $C_{0}(K, X)$ em $C_{0}(\Gamma, X)$. Então, para cada $y \in \Gamma$ e para cada $\eta>0$ o conjunto

$$
\left\{x \in K:\left|T^{*}\left(\varphi \cdot \delta_{y}\right)\right|(\{x\})>\eta \text { para algum } \varphi \in S_{X^{*}}\right\}
$$

é finito.

Demonstração. Por contradição assuma que para algum $\eta>0$ o conjunto

$$
\left\{x \in K:\left|T^{*}\left(\varphi \cdot \delta_{y}\right)\right|(\{x\})>\eta \text { para algum } \varphi \in S_{X^{*}}\right\}
$$

seja infinito.

Por hipótese, $X$ tem cotipo finito $2 \leq q<\infty$. Considere $Q>0$ exatamente como na demonstração da Proposição 4.7 e $m \in \mathbb{N}$ satisfazendo $\eta \cdot Q \cdot \sqrt[q]{m}>2\|T\|$. Fixe também pontos distintos $x_{1}, \ldots, x_{m} \in K$ e $\varphi_{1}, \ldots, \varphi_{m} \in S_{X^{*}}$ verificando

$$
\left\|T^{*}\left(\varphi_{i} \cdot \delta_{y}\right)\left(\left\{x_{i}\right\}\right)\right\|=\left|T^{*}\left(\varphi_{i} \cdot \delta_{y}\right)\right|\left(\left\{x_{i}\right\}\right)>\eta, 1 \leq i \leq m .
$$

Existem em $S_{X}$ vetores $v_{1}, \ldots, v_{m}$ satisfazendo

$$
\left\langle T^{*}\left(\varphi_{i} \cdot \delta_{y}\right)\left(\left\{x_{i}\right\}\right), v_{i}\right\rangle>\eta, 1 \leq i \leq m .
$$

Devido à regularidade das medidas $T^{*}\left(\varphi_{i} \cdot \delta_{y}\right), 1 \leq i \leq m$, podemos fixar $U_{1}, \ldots, U_{m}$, vizinhanças 
mutuamente disjuntas de $x_{1}, \ldots, x_{m}$ respectivamente, tais que

$$
\left|T^{*}\left(\varphi_{i} \cdot \delta_{y}\right)\right|\left(U_{i} \backslash\left\{x_{i}\right\}\right) \leq \frac{\eta}{2} .
$$

Aplicando o Lema de Urysohn 1.12 podemos encontrar funções $h_{i} \in C_{0}(K) \operatorname{com} 0 \leq h_{i}(x) \leq 1$ para todo $x \in K, h_{i}\left(x_{i}\right)=1$ e $h_{i}(x)=0$ se $x \in K \backslash U_{i}$. Defina $f_{i} \in C_{0}(K, X)$ por $f_{i}=v_{i} \cdot h_{i}$. Das relações $(4.5)$ e (4.6),

$$
\begin{aligned}
\left\|T f_{i}(y)\right\| & \geq\left|\left\langle\varphi_{i}, T f_{i}(y)\right\rangle\right|=\left|\int f_{i} d T^{*}\left(\varphi_{i} \cdot \delta_{y}\right)\right| \\
& \geq\left|\left\langle T^{*}\left(\varphi_{i} \cdot \delta_{y}\right)\left(\left\{x_{i}\right\}\right), v_{i}\right\rangle\right|-\left|\int f_{i} d T^{*}\left(\varphi_{i} \cdot \delta_{y}\right)-\left\langle T^{*}\left(\varphi_{i} \cdot \delta_{y}\right)\left(\left\{x_{i}\right\}\right), v_{i}\right\rangle\right| \\
& >\eta-\left|\int f_{i} d T^{*}\left(\varphi_{i} \cdot \delta_{y}\right)-\int_{x_{i}} f_{i} d T^{*}\left(\varphi_{i} \cdot \delta_{y}\right)\right| \\
& \geq \eta-\left|T^{*}\left(\varphi_{i} \cdot \delta_{y}\right)\right|\left(U_{i} \backslash\left\{x_{i}\right\}\right) \geq \frac{\eta}{2}
\end{aligned}
$$

Existe, como em 4.4, uma escolha apropriada de escalares $r_{i}= \pm 1$ verificando

$$
\left\|\sum_{i=1}^{m} r_{i} \cdot T f_{i}(y)\right\| \geq \frac{\eta \cdot Q \cdot \sqrt[q]{m}}{2}
$$

Em virtude de $U_{i} \cap U_{j}=\varnothing$, se $i \neq j$, e $\left\|f_{i}\right\| \leq 1,1 \leq i \leq m$, temos

$$
\left\|\sum_{i=1}^{m} r_{i} \cdot f_{i}\right\| \leq 1
$$

Por (4.7) e pela escolha de $m$,

$$
\|T\| \geq\left\|T\left(\sum_{i=1}^{m} r_{i} \cdot f_{i}\right)\right\| \geq\left\|T\left(\sum_{i=1}^{m} r_{i} \cdot f_{i}\right)(y)\right\|=\left\|\sum_{i=1}^{m} r_{i} \cdot T f_{i}(y)\right\|>\|T\|,
$$

o que é uma contradição.

\subsection{Cotas inferiores para as distâncias entre $C_{0}(K, X)$ e $C_{0}(K, X)$}

Nesta seção demonstraremos o principal resultado deste capítulo, o Teorema 4.2.

Demonstração do Teorema 4.2. A prova será obtida por redução ao absurdo em quatro passos.

Passo 1. Neste primeiro passo, assumindo a existência de um isomorfismo sobrejetor $T$ de $C_{0}(K, X)$ em $C_{0}(\Gamma, X)$ com distorção $\|T\|\left\|T^{-1}\right\|<2 n+1$, construiremos funções especiais $\alpha$ e $\beta$ em $C_{0}(\Gamma)$.

Sem perda de generalidade vamos supor que $\left\|T^{-1}\right\|=1$, do contrário podemos simplesmente substituir $T$ pelo isomorfismo $\left\|T^{-1}\right\| T$ que claramente possui esta propriedade. Desta forma, para cada $f \in C_{0}(K, X)$ temos $\|f\| \leq\|T f\|$. 
Sejam $0<\eta, \epsilon<1$ e tais que

$$
\|T\|<(2 n+1) \frac{1-\epsilon}{1+\epsilon} \quad \text { e } \quad \eta<\min \left\{\epsilon, \frac{(2 n+1)(1-\epsilon)-\|T\|}{2}\right\} .
$$

Fixe $v \in S_{X}$. Pela hipótese de $K^{(n)} \neq \varnothing$ existem pontos $x_{1}, \ldots, x_{n} \in K$, subconjuntos compactos $J_{1}, \ldots, J_{n} \subset K$, funções $h_{1}, \ldots, h_{n} \in C_{0}(K)$ e subconjuntos $G_{1}, \ldots, G_{n} \subset \Gamma$ verificando os itens (a), (b), (c) e (d) da Proposição 4.7. Para cada $1 \leq i \leq n$ considere

(a) $f_{i}=v \cdot h_{i} \in C_{0}(K, X)$,

(b) $g_{i}=\mathbb{1}_{G_{i}} \cdot T f_{i}$ onde $\mathbb{1}_{G_{i}}$ é a função característica de $G_{i}$.

Denote por $G$ o conjunto finito $\bigcup_{i=1}^{n} G_{i}$. De acordo com a Proposição 4.8

$$
H=\bigcup_{y \in G}\left\{x \in K:\left|T^{*}\left(\varphi \cdot \delta_{y}\right)\right|(\{x\}) \geq \eta \text { para algum } \varphi \in S_{X^{*}}\right\}
$$

é finito, então fixamos um ponto $z \in \stackrel{\circ}{J}_{n} \backslash H$. Seja $v^{*} \in S_{X^{*}}$ um funcional satisfazendo $\left\langle v^{*}, v\right\rangle=1 \mathrm{e}$ defina a seguinte medida vetorial

$$
\mu=\left(T^{-1}\right)^{*}\left(v^{*} \cdot \delta_{z}\right)
$$

De acordo com o Teorema 1.22 existe uma função $\gamma: \Gamma \rightarrow X^{*}$ tal que $\|\gamma(y)\|=1$ para todo $y \in \Gamma \mathrm{e}$

$$
\langle\mu(A), u\rangle=\int_{A}\langle\gamma(y), u\rangle d|\mu|(y), A \subset \Gamma \text { e } u \in X .
$$

Mais ainda, analogamente como na Proposição 1.23, demonstra-se que

$$
\int f d \mu=\int\langle\gamma(y), f(y)\rangle d|\mu|(y), f \in C_{0}(\Gamma, X)
$$

Por $\|\gamma(y)\|=1, y \in \Gamma$, e pela escolha do ponto $z$ temos

$$
\left|T^{*}\left(\gamma(y) \cdot \delta_{y}\right)\right|(\{z\})<\eta, y \in G .
$$

Por regularidade, para cada $y \in G$ existe uma vizinhança aberta de $z, U_{y} \subset J_{n}$, tal que $\left|T^{*}\left(\gamma(y) \cdot \delta_{y}\right)\right|\left(U_{y}\right)<\eta$. Fixe $U=\bigcap_{y \in G} U_{y}$.

Aplicando o Lema de Urysohn 1.12, podemos encontrar uma função $h_{n+1} \in C_{0}(K)$ tal que $0 \leq h_{n+1}(x) \leq 1$ para cada $x \in K, h_{n+1}(z)=1$ e $h_{n+1}(x)=0$ se $x \in K \backslash U$. Seja $f_{n+1}=v \cdot h_{n+1} \in$ $C_{0}(K, X)$ e defina funções $\alpha, \beta \in C_{0}(\Gamma)$, em cada $y \in \Gamma$, por

$$
\begin{aligned}
& \alpha(y)=\left\langle\gamma(y), T f_{n+1}(y)\right\rangle \\
& \beta(y)=\left\langle\gamma(y), g_{1}(y)+2 \sum_{i=2}^{n} g_{i}(y)+2 T f_{n+1}(y)\right\rangle .
\end{aligned}
$$


Passo 2. Vamos demonstrar que $\|\beta\|=\max \{2\|\alpha\|,|\beta(y)|: y \in G\}$.

Para cada $y \in G$,

$$
\begin{aligned}
|\alpha(y)| & =\left|\left\langle\gamma(y), T f_{n+1}(y)\right\rangle\right|=\left|\int T f_{n+1} d\left(\gamma(y) \cdot \delta_{y}\right)\right| \\
& =\left|\int f_{n+1} d T^{*}\left(\gamma(y) \cdot \delta_{y}\right)\right| \leq\left|T^{*}\left(\gamma(y) \cdot \delta_{y}\right)\right|(U)<\eta<1 .
\end{aligned}
$$

Seja $y_{0} \in \Gamma$ tal que $\|\alpha\|=\left|\alpha\left(y_{0}\right)\right|$. Pela relação (4.8) e por $|\mu|(\Gamma)=\left\|\left(T^{-1}\right)^{*}\left(v^{*} \cdot \delta_{z}\right)\right\| \leq 1$,

$$
\begin{aligned}
\left|\alpha\left(y_{0}\right)\right| & =\left|\left\langle\gamma\left(y_{0}\right), T f_{n+1}\left(y_{0}\right)\right\rangle\right| \geq\left|\int\left\langle\gamma(y), T f_{n+1}(y)\right\rangle d\right| \mu|(y)| \\
& =\left|\int T f_{n+1} d \mu\right|=\left|\int T f_{n+1} d\left(T^{-1}\right)^{*}\left(v^{*} \cdot \delta_{z}\right)\right|=\left|\int f_{n+1} d\left(v^{*} \cdot \delta_{z}\right)\right| \\
& =\left|\left\langle v^{*}, f_{n+1}(z)\right\rangle\right|=\left\langle v^{*}, v\right\rangle=1 .
\end{aligned}
$$

Portanto $y_{0} \in \Gamma \backslash G$ e como $\beta(y)=2 \alpha(y)$ para todo $y \in \Gamma \backslash G$, o Passo 2 está verificado.

Passo 3. Vamos demonstrar que $\|\beta\| \geq(2 n+1)-(2 n-1) \epsilon$.

Seja $y_{0}$ tal que $\|\beta\|=\left|\beta\left(y_{0}\right)\right|$. Utilizando a relação (4.8), podemos escrever

$$
\begin{aligned}
\left|\beta\left(y_{0}\right)\right| & =\left|\left\langle\gamma\left(y_{0}\right), g_{1}\left(y_{0}\right)+2 \sum_{i=2}^{n} g_{i}\left(y_{0}\right)+2 T f_{n+1}\left(y_{0}\right)\right\rangle\right| \\
& \geq\left|\int\left\langle\gamma(y), g_{1}(y)+2 \sum_{i=2}^{n} g_{i}(y)+2 T f_{n+1}(y)\right\rangle d\right| \mu|(y)| \\
& =\left|\int g_{1}+2 \sum_{i=2}^{n} g_{i}+2 T f_{n+1} d T^{-1^{*}}\left(v^{*} \cdot \delta_{z}\right)\right| \\
& =\left|\left\langle v^{*}, T^{-1} g_{1}(z)+2 \sum_{i=2}^{n} T^{-1} g_{i}(z)+2 f_{n+1}(z)\right\rangle\right| \\
& \geq\left|\left\langle v^{*}, f_{1}(z)+2 \sum_{i=2}^{n+1} f_{i}(z)\right\rangle\right|-\left|\left\langle v^{*}, f_{1}(z)-T^{-1} g_{1}(z)\right\rangle\right| \\
& -2 \sum_{i=2}^{n}\left|\left\langle v^{*}, f_{i}(z)-T^{-1} g_{i}(z)\right\rangle\right| .
\end{aligned}
$$

Como $\|f\| \leq\|T f\|, f \in C_{0}(K, X)$, temos

$$
\left|\left\langle v^{*}, f_{i}(z)-T^{-1} g_{i}(z)\right\rangle\right| \leq\left\|f_{i}-T^{-1} g_{i}\right\| \leq\left\|T f_{i}-g_{i}\right\|=\left\|\left(1-\mathbb{1}_{G_{i}}\right) \cdot T f_{i}\right\| \leq \epsilon, 1 \leq i \leq n .
$$

Mais ainda, pela definição das funcões $f_{i}$, vale

$$
\left\langle v^{*}, f_{i}(z)\right\rangle=\left\langle v^{*}, v\right\rangle=1,1 \leq i \leq n+1 .
$$

Portanto,

$$
\|\beta\| \geq(2 n+1)-(2 n-1) \epsilon .
$$


Passo 4. Em virtude de $\|\beta\| \geq(2 n+1)-(2 n-1) \epsilon$, de acordo com o Passo 2, existem duas possibilidades:

(i) $2\|\alpha\| \geq(2 n+1)-(2 n-1) \epsilon$,

(ii) $|\beta(y)| \geq(2 n+1)-(2 n-1) \epsilon$ para algum $y \in G$.

Mostraremos que ambas as possibilidades implicam uma contradição.

Suponha inicialmente que $(i)$ seja verdadeira. Defina

$$
A=T^{-1} g_{1}-2 f_{n+1} \text {. }
$$

Recordando que $0 \leq h_{n+1} \leq h_{1} \leq 1$ e que $\|f\| \leq\|T f\|, f \in C_{0}(K, X)$, temos para cada $x \in K$,

$$
\begin{aligned}
\left\|T^{-1} g_{1}(x)-2 f_{n+1}(x)\right\| & \leq\left\|f_{1}(x)-2 f_{n+1}(x)\right\|+\left\|T^{-1} g_{1}(x)-f_{1}(x)\right\| \\
& \leq\left\|v \cdot h_{1}(x)-2 v \cdot h_{n+1}(x)\right\|+\left\|T^{-1} g_{1}-f_{1}\right\| \\
& \leq\left|h_{1}(x)-2 h_{n+1}(x)\right|+\left\|g_{1}-T f_{1}\right\| \leq 1+\epsilon .
\end{aligned}
$$

Portanto $\|A\| \leq 1+\epsilon$.

Devido a (4.9) e (4.10) existe $y_{0} \in \Gamma \backslash G$ tal que $\|\alpha\|=\left|\alpha\left(y_{0}\right)\right|$. Podemos escrever

$$
\begin{aligned}
\left|\left\langle\gamma\left(y_{0}\right), T(A)\left(y_{0}\right)\right\rangle\right| & =2\left|\left\langle\gamma\left(y_{0}\right), T f_{n+1}\left(y_{0}\right)\right\rangle\right|=2\|\alpha\| \\
& \geq(2 n+1)-(2 n-1) \epsilon>(2 n+1)(1-\epsilon) .
\end{aligned}
$$

Consequentemente,

$$
\|T\| \geq\left\|T\left(\frac{1}{1+\epsilon} A\right)\right\|>(2 n+1) \frac{1-\epsilon}{1+\epsilon} .
$$

Isto contradiz a escolha de $\epsilon$.

Em seguida, assuma que (ii) seja verdadeiro. Distinguimos dois casos.

Caso 1. $\|\beta\|=\left|\beta\left(y_{0}\right)\right|$ para algum $y_{0} \in G_{1}$. Neste caso, por $G_{1}, \ldots, G_{n}$ serem mutuamente disjuntos, temos

$$
\left|\beta\left(y_{0}\right)\right|=\left|\left\langle\gamma\left(y_{0}\right), g_{1}\left(y_{0}\right)+2 T f_{n+1}\left(y_{0}\right)\right\rangle\right|>(2 n+1)-(2 n-1) \epsilon .
$$

Pela escolha de $\eta$,

$$
\begin{aligned}
\left|\left\langle\gamma\left(y_{0}\right), g_{1}\left(y_{0}\right)\right\rangle\right| & \geq(2 n+1)-(2 n-1) \epsilon-2\left|\left\langle\gamma\left(y_{0}\right), T f_{n+1}\left(y_{0}\right)\right\rangle\right| \\
& >(2 n+1)-(2 n-1) \epsilon-2 \eta>\|T\| .
\end{aligned}
$$

Consequentemente,

$$
\|T\| \geq\left\|T f_{1}\right\| \geq\left|\left\langle\gamma\left(y_{0}\right), T f_{1}\left(y_{0}\right)\right\rangle\right|=\left|\left\langle\gamma\left(y_{0}\right), g_{1}\left(y_{0}\right)\right\rangle\right|>\|T\|,
$$

o que é uma contradição.

Caso 2. $\|\beta\|=\left|\beta\left(y_{0}\right)\right|$ para algum $y_{0} \in G_{i}, i>1$. Mais uma vez, por $G_{1}, \ldots, G_{n}$ serem mutuamente disjuntos, temos

$$
\left|\beta\left(y_{0}\right)\right|=\left|\left\langle\gamma\left(y_{0}\right), 2 g_{i}\left(y_{0}\right)+2 T f_{n+1}\left(y_{0}\right)\right\rangle\right| \geq(2 n+1)-(2 n-1) \epsilon .
$$


Recordando que $\eta<\epsilon$,

$$
\begin{aligned}
2\left|\left\langle\gamma\left(y_{0}\right), g_{i}\left(y_{0}\right)\right\rangle\right| & \geq(2 n+1)-(2 n-1) \epsilon-2\left|\left\langle\gamma\left(y_{0}\right), T f_{n+1}\left(y_{0}\right)\right\rangle\right| \\
& >(2 n+1)(1-\epsilon) .
\end{aligned}
$$

Considere a função

$$
B_{i}=T^{-1} g_{1}-2 f_{i}
$$

Da relação (4.11) e por $\left\|\gamma\left(y_{0}\right)\right\|=1$,

$$
\begin{aligned}
\left\|T\left(B_{i}\right)\right\| & \geq\left|\left\langle\gamma\left(y_{0}\right), T\left(B_{i}\right)\left(y_{0}\right)\right\rangle\right|=2\left|\left\langle\gamma\left(y_{0}\right), T f_{i}\left(y_{0}\right)\right\rangle\right| \\
& =2\left|\left\langle\gamma\left(y_{0}\right), g_{i}\left(y_{0}\right)\right\rangle\right|>(2 n+1)(1-\epsilon) .
\end{aligned}
$$

Por outro lado, lembrando que $0 \leq h_{i} \leq h_{1} \leq 1$ e que $\|f\| \leq\|T f\|, f \in C_{0}(K, X)$, temos, para cada $x \in K$,

$$
\begin{aligned}
\left\|T^{-1} g_{1}(x)-2 f_{i}(x)\right\| & \leq\left\|f_{1}(x)-2 f_{i}(x)\right\|+\left\|T^{-1} g_{1}(x)-f_{1}(x)\right\| \\
& \leq\left\|v \cdot h_{1}(x)-2 v \cdot h_{i}(x)\right\|+\left\|T^{-1} g_{1}-f_{1}\right\| \\
& \leq\left|h_{1}(x)-2 h_{i}(x)\right|+\left\|g_{1}-T f_{1}\right\| \leq 1+\epsilon .
\end{aligned}
$$

Portanto $\left\|B_{i}\right\| \leq 1+\epsilon$ e deduzimos

$$
\|T\| \geq\left\|T\left(\frac{1}{1+\epsilon} B_{i}\right)\right\|>(2 n+1) \frac{1-\epsilon}{1+\epsilon}
$$

o que contradiz a escolha de $\epsilon$.

\subsection{Cotas superiores as para distâncias entre $C\left(\left[1, \omega^{n} k\right], X\right)$ e $C_{0}(\mathbb{N}, X)$}

Nesta seção vamos mostrar como generalizar a fórmula do isomorfismo (4.1) mencionado na introdução. Nosso objetivo é obter cotas superiores para as distâncias de Banach-Mazur entre os espaços $C_{0}(\mathbb{N}, X)$ e $C\left(\left[1, \omega^{n} k\right], X\right), 1 \leq k, n<\omega$, onde $X$ denota um espaço de Banach arbitrário.

Para simplificar nossa notação vamos introduzir uma nova terminologia. Recordamos que cada número ordinal $1 \leq \xi<\omega^{\omega}$ admite uma única representação na forma normal de Cantor, ver [47, p. 153],

$$
\xi=\omega^{n_{k}} m_{k}+\ldots+\omega^{n_{2}} m_{2}+\omega^{n_{1}} m_{1}
$$

onde $0 \leq n_{1}<n_{2}<\ldots<n_{k}<\omega$ e $1 \leq m_{1}<\omega, 1 \leq m_{2}<\omega, \ldots, 1 \leq m_{k}<\omega$ e $1 \leq k<\omega$.

Definição 4.9. Para um número ordinal $1 \leq \xi<\omega^{\omega}$, representado na forma normal de Cantor como em (4.12), definimos $\xi^{[0]}=\xi$ e por indução

$$
\xi^{[r]}= \begin{cases}\omega^{n_{k}} m_{k}+\ldots+\omega^{n_{2}} m_{2}+\omega^{n_{1}+1} & \text { se } r=1, \\ \left(\xi^{[r-1]}\right)^{[1]} & \text { se } 1 \leq r<\omega .\end{cases}
$$

Observação 4.10. Utilizando a forma normal de Cantor é fácil verificar que cada número ordinal $1 \leq \xi<\omega^{n}$ admite uma única representação na forma

$$
\xi=\omega^{n-1} i_{1}+\omega^{n-2} i_{2}+\ldots+\omega^{n-(j-1)} i_{j-1}+\omega^{n-j} i_{j}
$$

onde $1 \leq j \leq n, 1 \leq i_{j}<\omega$ e $0 \leq i_{r}<\omega$ se $1 \leq r \leq j-1$. 
Essa representação alternativa é mais conveniente para a função $\xi \mapsto \xi^{[1]}$ apresentada na Definição 4.9. Se $1 \leq \xi<\omega^{n}$ é um ordinal representado na forma (4.13), então

$$
\begin{aligned}
& \xi^{[1]}=\omega^{n-1} i_{1}+\omega^{n-2} i_{2}+\ldots+\omega^{n-(j-1)}\left(i_{j-1}+1\right) \\
& \xi^{[2]}=\omega^{n-1} i_{1}+\omega^{n-2} i_{2}+\ldots+\omega^{n-(j-2)}\left(i_{j-2}+1\right) \\
& \quad \vdots \\
& \xi^{[j-2]}=\omega^{n-1} i_{1}+\omega^{n-2}\left(i_{2}+1\right) \\
& \xi^{[j-1]}=\omega^{n-1}\left(i_{1}+1\right) \\
& \xi^{[j]}=\omega^{n}
\end{aligned}
$$

Lema 4.11. Sejam $1 \leq n<\omega, A$ e $B$ números reais e $X$ um espaço de Banach. Para cada $f \in C\left(\left[1, \omega^{n}\right], X\right)$ considere a sequência $\left(a_{\xi}\right)_{1 \leq \xi \leq \omega^{n} \text {, }}$

$$
a_{\xi}= \begin{cases}A & \text { se } \xi=\omega^{n} \\ B\left(f(\xi)-f\left(\xi^{[1]}\right)\right) & \text { se } 1 \leq \xi<\omega^{n} .\end{cases}
$$

Então, para cada $\epsilon>0$ existe somente um número finito de ordinais $1 \leq \xi \leq \omega^{n}$ satisfazendo $\left\|a_{\xi}\right\| \geq \epsilon$.

Demonstração. Será feita por indução em $n$. Naturalmente o lema é verdadeiro para $n=1$. Assuma ser verdadeiro para $n-1$, com $n \geq 2$, fixe $f \in C\left(\left[1, \omega^{n}\right], X\right)$ arbitrário e considere a sequência $\left(a_{\xi}\right)_{1 \leq \xi \leq \omega^{n}}$ como acima.

Dado $\epsilon>0$ arbitrário, da continuidade de $f$, existe $1 \leq m<\omega$ tal que

$$
\left.\xi \in] \omega^{n-1} m, \omega^{n}\right] \Longrightarrow\left\|f(\xi)-f\left(\omega^{n}\right)\right\|<\frac{\epsilon}{2(|B|+1)} .
$$

Se $\xi \in] \omega^{n-1} m, \omega^{n}\left[\right.$, então $\left.\left.\xi^{[1]} \in\right] \omega^{n-1} m, \omega^{n}\right]$ e portanto

$$
\begin{aligned}
\left\|a_{\xi}\right\| & =|B|\left\|f(\xi)-f\left(\xi^{[1]}\right)\right\| \leq|B|\left(\left\|f(\xi)-f\left(\omega^{n}\right)\right\|+\left\|f\left(\xi^{[1]}\right)-f\left(\omega^{n}\right)\right\|\right) \\
& <\frac{|B| \epsilon}{2(|B|+1)}+\frac{|B| \epsilon}{2(|B|+1)}<\epsilon .
\end{aligned}
$$

Para cada $1 \leq r \leq m$ defina $g_{r} \in C\left(\left[1, \omega^{n-1}\right], X\right)$ como

$$
g_{r}(\xi)=f\left(\omega^{n-1}(r-1)+\xi\right), 1 \leq \xi \leq \omega^{n-1} .
$$

Em seguida, fixe a sequência $\left(a_{\xi}^{r}\right)_{1 \leq \xi \leq \omega^{n-1}}$

$$
a_{\xi}^{r}= \begin{cases}A & \text { se } \xi=\omega^{n-1} \\ B\left(g_{r}(\xi)-g_{r}\left(\xi^{[1]}\right)\right) & \text { se } 1 \leq \xi<\omega^{n-1} .\end{cases}
$$

De acordo com a hipótese de indução existe somente um número finito de ordinais $1 \leq \xi \leq \omega^{n-1}$ satisfazendo $\left\|a_{\xi}^{r}\right\| \geq \epsilon$. Por construção, temos

$$
a_{\xi}^{r}=a_{\omega^{n-1}(r-1)+\xi}, \quad 1 \leq \xi<\omega^{n-1} .
$$

Deduzimos que para cada $1 \leq r \leq m$ existe somente um número finito de ordinais $\xi$ no intervalo $\left[\omega^{n-1}(r-1)+1, \omega^{n-1} r\right]$ verificando $\left\|a_{\xi}\right\| \geq \epsilon$. Como $\left[1, \omega^{n}\right]$ é a união dos intervalos $\left[1, \omega^{n-1}\right], \ldots,\left[\omega^{n-1}(m-1)+1, \omega^{n-1} m\right]$ e $\left[\omega^{n-1} m+1, \omega^{n}\right]$, o lema está demonstrado. 
Demonstração do Teorema 4.3. De início, observe que

$$
\begin{aligned}
& C\left(\left[1, \omega^{n} k\right], X\right)=\underbrace{C\left(\left[1, \omega^{n}\right], X\right) \oplus \ldots \oplus C\left(\left[1, \omega^{n}\right], X\right)}_{k}, \\
& C_{0}(\mathbb{N}, X)=\underbrace{C_{0}(\mathbb{N}, X) \oplus \ldots \oplus C_{0}(\mathbb{N}, X)}_{k} .
\end{aligned}
$$

Então, para estabelecer o Teorema 4.3 é suficiente demonstrar que

$$
\mathrm{d}\left(C\left(\left[1, \omega^{n}\right], X\right), C_{0}(\mathbb{N}, X)\right) \leq 2 n+1 .
$$

Para tanto, vamos construir um isomorfismo sobrejetor $T: C\left(\left[1, \omega^{n}\right], X\right) \rightarrow C_{0}(\mathbb{N}, X)$ com distorção $\|T\|\left\|T^{-1}\right\|=2 n+1$.

Seja $\Gamma_{n}$ o intervalo de ordinal $\left[1, \omega^{n}\right]$ munido da topologia discreta. Para simplificar a demonstração vamos substituir $C_{0}(\mathbb{N}, X)$ por $C_{0}\left(\Gamma_{n}, X\right)$. Estes espaços são isometricamente isomorfos.

Para cada $f \in C\left(\left[1, \omega^{n}\right], X\right)$ defina a função $T(f): \Gamma_{n} \rightarrow X$ por

$$
T(f)(\xi)= \begin{cases}2 f\left(\omega^{n}\right) & \text { se } \xi=\omega^{n} \\ f(\xi)-f\left(\xi^{[1]}\right) & \text { se } 1 \leq \xi<\omega^{n}\end{cases}
$$

Segue imediatamente por uma aplicação do Lema 4.11, com $A=2 f\left(\omega^{n}\right)$ e $B=1$, que $T(f) \in$ $C_{0}\left(\Gamma_{n}, X\right)$ para todo $f \in C\left(\left[1, \omega^{n}\right], X\right)$. Portanto, $T$ define uma aplicação de $C\left(\left[1, \omega^{n}\right], X\right)$ em $C_{0}\left(\Gamma_{n}, X\right)$. Para verificar que $T$ é um operador linear fixe $f_{1}, f_{2} \in C_{0}\left(\Gamma_{n}, X\right)$ e um escalar $\lambda$ arbitrário. Temos

$$
\begin{aligned}
T\left(\lambda f_{1}+f_{2}\right)\left(\omega^{n}\right) & =2\left(\lambda f_{1}+f_{2}\right)\left(\omega^{n}\right)=\lambda\left(2 f_{1}\left(\omega^{n}\right)\right)+2 f_{2}\left(\omega^{n}\right) \\
& =\lambda T\left(f_{1}\right)\left(\omega^{n}\right)+T\left(f_{2}\right)\left(\omega^{n}\right) .
\end{aligned}
$$

Se $1 \leq \xi<\omega^{n}$, então

$$
\begin{aligned}
T\left(\lambda f_{1}+f_{2}\right)(\xi) & =\left(\lambda f_{1}+f_{2}\right)(\xi)-\left(\lambda f_{1}+f_{2}\right)\left(\xi^{[1]}\right) \\
& =\lambda\left(f_{1}(\xi)-f_{1}\left(\xi^{[1]}\right)\right)+\left(f_{2}(\xi)-f_{2}\left(\xi^{[1]}\right)\right) \\
& =\lambda T\left(f_{1}\right)(\xi)+T\left(f_{2}\right)(\xi) .
\end{aligned}
$$

Deduzimos que $T$ é um operador linear de $C\left(\left[1, \omega^{n}\right], X\right)$ em $C_{0}\left(\Gamma_{n}, X\right)$. Esse operador claramente verifica $\|T\|=2$.

Em seguida, para cada função $g \in C_{0}\left(\Gamma_{n}, X\right)$, recordando a Observação 4.10, defina a função $S(g):\left[1, \omega^{n}\right] \rightarrow X$ por

$$
S(g)(\xi)= \begin{cases}\frac{1}{2} g\left(\omega^{n}\right) & \text { se } \xi=\omega^{n}, \\ g(\xi)+g\left(\xi^{[1]}\right)+\ldots+g\left(\xi^{[j-2]}\right)+g\left(\xi^{[j-1]}\right)+\frac{1}{2} g\left(\xi^{[j]}\right) & \text { se } 1 \leq \xi<\omega^{n} \text { é como (4.13). }\end{cases}
$$

Vamos demonstrar que $S(g)$ é contínua em $\left[1, \omega^{n}\right]$ para cada $g \in C_{0}\left(\Gamma_{n}, X\right)$. Para tanto, seja $g \in C_{0}\left(\Gamma_{n}, X\right)$ arbitrário. Dado $\xi_{0}$ um ponto não isolado de $\left[1, \omega^{n}\right]$ fixe $\epsilon>0$ e defina

$$
\Lambda_{\epsilon}=\left\{1 \leq \xi \leq \omega^{n}:\|g(\xi)\| \geq \frac{\epsilon}{n}\right\}
$$

Para benefício do leitor distinguimos dois casos.

Caso 1. $\xi_{0}=\omega^{n}$. Por $\Lambda_{\epsilon}$ ser finito existe $1 \leq m<\omega$ tal que

$$
] \omega^{n-1} m, \omega^{n}\left[\cap \Lambda_{\epsilon}=\varnothing\right.
$$


Segue da definição de $S(g)$ que se $\xi \in] \omega^{n-1} m, \omega^{n}[$, então

$$
\left\|S(g)(\xi)-S(g)\left(\omega^{n}\right)\right\| \leq\left\|g\left(\xi_{1}\right)\right\|+\ldots+\left\|g\left(\xi_{s}\right)\right\|
$$

onde $1 \leq s \leq n$ e $\xi=\xi_{1}<\ldots<\xi_{s}<\xi_{0}$. Logo,

$$
\left\|S(g)(\xi)-S(g)\left(\omega^{n}\right)\right\|<\epsilon .
$$

Caso 2. $1 \leq \xi_{0}<\omega^{n}$. Escreva $\xi_{0}=\omega^{n-1} i_{1}+\ldots+\omega^{n-j} i_{j}$ com $1 \leq j<n, 1 \leq i_{j}<\omega$ e $0 \leq i_{p}<\omega$ se $1 \leq p \leq j-1$. Por $\Lambda_{\epsilon}$ ser finito existe $1 \leq m<\omega$ tal que

$$
] \omega^{n-1} i_{1}+\ldots+\omega^{n-j}\left(i_{j}-1\right)+\omega^{n-(j+1)} m, \omega^{n-1} i_{1}+\ldots+\omega^{n-j} i_{j}\left[\cap \Lambda_{\epsilon}=\varnothing .\right.
$$

Observe que se

$$
\xi \in] \omega^{n-1} i_{1}+\ldots+\omega^{n-j}\left(i_{j}-1\right)+\omega^{n-(j+1)} m, \omega^{n-1} i_{1}+\ldots+\omega^{n-j} i_{j}[,
$$

então existe $1 \leq s \leq n-j$ tal que $\xi^{[s]}=\xi_{0}$. Temos, pela definição de $S(g)$,

$$
\left\|S(g)(\xi)-S(g)\left(\xi_{0}\right)\right\| \leq\left\|g\left(\xi_{1}\right)\right\|+\ldots+\left\|g\left(\xi_{s}\right)\right\|
$$

onde $\xi=\xi_{1}<\ldots<\xi_{s}<\xi_{0}$. Consequentemente

$$
\left\|S(g)(\xi)-S(g)\left(\xi_{0}\right)\right\|<\epsilon .
$$

Deduzimos que $S(g), g \in C_{0}\left(\Gamma_{n}, X\right)$, é contínua em $\left[1, \omega^{n}\right]$. Assim, $S$ define uma aplicação de $C_{0}\left(\Gamma_{n}, X\right)$ em $C\left(\left[1, \omega^{n}\right], X\right)$.

Vamos verificar que as aplicações $S \circ T$ e $T \circ S$ são, respectivamente, os operadores identidade em $C\left(\left[1, \omega^{n}\right], X\right)$ e $C_{0}\left(\Gamma_{n}, X\right)$.

Com efeito, seja $f \in C\left(\left[1, \omega^{n}\right], X\right)$ arbitrário. Se $\xi=\omega^{n}$ então

$$
S \circ T(f)\left(\omega^{n}\right)=\frac{1}{2} T(f)\left(\omega^{n}\right)=f\left(\omega^{n}\right) .
$$

Seja $1 \leq \xi<\omega^{n}$ representado na forma (4.13). Das definições de $S$ e $T$ podemos escrever

$$
\begin{aligned}
S \circ T(f)(\xi) & =\sum_{r=0}^{j-1} T(f)\left(\xi^{[r]}\right)+\frac{1}{2} T(f)\left(\xi^{[j]}\right)=\sum_{r=0}^{j-1}\left(f\left(\xi^{[r]}\right)-f\left(\xi^{[r+1]}\right)\right)+f\left(\omega^{n}\right) \\
& =\left(f(\xi)-f\left(\omega^{n}\right)\right)+f\left(\omega^{n}\right)=f(\xi) .
\end{aligned}
$$

Concluímos que $S \circ T(f)=f$ para cada $f \in C\left(\left[1, \omega^{n}\right], X\right)$.

Por outro lado, fixe $g \in C_{0}\left(\Gamma_{n}, X\right)$ arbitrário. Analogamente como em (4.16) demonstra-se que

$$
T \circ S(g)\left(\omega^{n}\right)=g\left(\omega^{n}\right) .
$$

Fixe $1 \leq \xi<\omega^{n}$ representado na forma (4.13). Das definições de $T$ e $S$ temos

$$
T \circ S(g)(\xi)=S(g)(\xi)-S(g)\left(\xi^{[1]}\right)=\left(\sum_{r=0}^{j-1} g\left(\xi^{[r]}\right)+\frac{1}{2} g\left(\xi^{[j]}\right)\right)-\left(\sum_{r=1}^{j-1} g\left(\xi^{[r]}\right)+\frac{1}{2} g\left(\xi^{[j]}\right)\right)=g(\xi) .
$$

Fica estabelecido que $T \circ S(g)=g$ para cada $g \in C_{0}\left(\Gamma_{n}, X\right)$ e $S \circ T(f)=f$ para cada 
$f \in C\left(\left[1, \omega^{n}\right], X\right)$, ou seja, $S$ é o operador inverso de $T$, mais ainda, $S$ é linear e verifica

$$
\|S\|=\frac{2 n+1}{2} .
$$

Portanto, $T$ é um isomorfismo sobrejetor de $C\left(\left[1, \omega^{n}\right], X\right)$ em $C_{0}\left(\Gamma_{n}, X\right)$ com distorção

$$
\|T\|\left\|T^{-1}\right\|=2 n+1 .
$$

Isto completa nossa demonstração. 


\section{Capítulo 5}

\section{Sobre distâncias de Banach-Mazur entre espaços $C(\omega)$ e $C(\alpha), \omega \leq \alpha<\omega^{\omega}$}

\subsection{Introdução}

Para um ordinal $\alpha$, denotamos por $C(\alpha)$ o espaço das funções contínuas no intervalo de ordinal $[1, \alpha], \omega \leq \alpha<\omega_{1}$. A classificação isomorfa destes espaços é devida a Bessaga e Pełczyński [7]. Eles estabeleceram que se $\omega \leq \alpha \leq \beta<\omega_{1}$, então $C(\alpha)$ é isomorfo a $C(\beta)$ se e somente se existe $1 \leq n<\omega$ tal que $\alpha^{n} \leq \beta<\alpha^{n+1}$. Na ocasião, também foram apresentadas estimativas para as distâncias de Banach-Mazur entre estes espaços:

$$
n \leq \mathrm{d}(C(\alpha), C(\beta)) \leq 4^{n+3} .
$$

Em [7, p. 59], os autores consideram o problema de se obter funções $G, H: \mathbb{N} \rightarrow \mathbb{R}^{+}$satisfazendo

$$
\sup (H(n) / G(n))<\infty \quad \text { e } \quad G(n) \leq \mathrm{d}(C(\alpha), C(\beta)) \leq H(n), n \in \mathbb{N} .
$$

Neste capítulo, apresentaremos estimativas como acima para o caso $\alpha=\omega$. Estaremos focados nas distâncias entre os espaços $C(\omega)$ e $C\left(\omega^{n} k\right), 1 \leq n, k<\omega$, e neste sentido, obtemos as seguintes funções $G(n, k)$ e $H(n, k)$ que fornecem respectivamente cotas inferiores e superiores para as distâncias entre os espaços $C(\omega)$ e $C\left(\omega^{n} k\right)$,

$$
G(n, k)= \begin{cases}1 & \text { se } n=1, k=1 \\ 3 & \text { se } n=1, k>1 \\ 2 n-1 & \text { se } n>1, k=1 \\ 2 n+1 & \text { se } n>1, k>1\end{cases}
$$

$\mathrm{e}$

$$
H(n, k)= \begin{cases}1 & \text { se } n=1, k=1 \\ 2+\sqrt{5} & \text { se } n=1, k>1 \\ n+\sqrt{(n-1)(n+3)} & \text { se } n>1, k=1 \\ n+1+\sqrt{n(n+4)} & \text { se } n>1, k>1\end{cases}
$$

Estas funções também verificam:

$$
H(n, k)-G(n, k)<2,1 \leq n, k<\omega .
$$

Tendo em mente as técnicas desenvolvidas neste trabalho, as funções $H(n, k)$ e $G(n, k)$ sugerem a seguinte conjectura sobre os valores exatos dessas distâncias. 
Conjectura 5.1. Sejam $1<n, k<\omega$. Então

(a) $\mathrm{d}(C(\omega), C(\omega k))$ é igual a 3 .

(b) $\mathrm{d}\left(C(\omega), C\left(\omega^{n}\right)\right)$ é igual a $2 n-1,2 n$ ou $n+\sqrt{(n-1)(n+3)}$.

(c) $\mathrm{d}\left(C(\omega), C\left(\omega^{n} k\right)\right)$ é igual a $2 n+1,2 n+2$ ou $n+1+\sqrt{n(n+4)}$.

Observação 5.2. Para $n=2$ e $k=1$ temos

$$
3 \leq \mathrm{d}\left(C(\omega), C\left(\omega^{2}\right)\right) \leq 2+\sqrt{5} .
$$

Seria muito interessante estabelecer o valor exato desta distância. Se d $\left(C(\omega), C\left(\omega^{2}\right)\right)=3$, então o número 3 é a melhor constante possível no Teorema 3.4. Por outro lado, se $\mathrm{d}\left(C(\omega), C\left(\omega^{2}\right)\right)=2+\sqrt{5}$, temos uma resposta para um problema sugerido por A. Pełczyński em 1968, ver [42, Problema 28, p. 73], que até o presente momento permanece sem resposta. Em nossa notação, o referido problema pode ser descrito como se segue:

Problema 5.3 (Pełczyński). Sejam $K_{1}$ e $K_{2}$ espaços topológicos compactos de Hausdorff. Se $C\left(K_{1}\right) \sim C\left(K_{2}\right)$ então d $\left(C\left(K_{1}\right), C\left(K_{2}\right)\right)$ é um número inteiro?

Organizamos este capítulo do seguinte modo. Na Seção 5.2 vamos apresentar alguns resultados preliminares envolvendo espaços de Banach $C(K)$ onde $K$ é compacto de Hausdorff. Na Seção 5.3, recordando a Definição 3.6, demonstraremos o seguinte resultado:

Teorema 5.4. Sejam $1 \leq n<\omega, L$ um espaço compacto de Hausdorff infinito tal que $L^{(2)}=\varnothing e$ $K$ um compacto Hausdorff. Então

$$
C(K) \sim C(L) \text { e }\left|K^{(n)}\right|>\left|L^{(1)}\right| \Longrightarrow \mathrm{d}(C(K), C(L)) \geq 2 n+1 .
$$

Por consequência do Teorema 5.4, em virtude de $\left(\left[1, \omega^{n} k\right]\right)^{(n)}=\left\{\omega^{n}, \omega^{n} 2, \ldots, \omega^{n} k\right\}, 1 \leq n, k<\omega$, temos cotas inferiores para distâncias entre os espaços $C\left(\omega^{n} k\right)$ e $C(\omega)$.

Corolário 5.5. Sejam $1<n, k<\omega$. Então

(a) $\mathrm{d}(C(\omega), C(\omega k)) \geq 3$.

(b) $\mathrm{d}\left(C(\omega), C\left(\omega^{n}\right)\right) \geq 2 n-1$.

(c) $\mathrm{d}\left(C(\omega), C\left(\omega^{n} k\right)\right) \geq 2 n+1$.

Na Seção 5.4 vamos obter cotas superiores para distâncias entre os espaços $C\left(\omega^{n} k\right), 1 \leq n, k<\omega$, e $C(\omega)$. Nossa tarefa é construir isomorfismos sobrejetores de $C\left(\omega^{n} k\right)$ em $C(\omega)$ mantendo algum controle sobre as distorções. Neste sentido demonstramos:

Teorema 5.6. Sejam $1<n, k<\omega$. Então

(a) $\mathrm{d}(C(\omega), C(\omega k)) \leq 2+\sqrt{5}$.

(b) $\mathrm{d}\left(C(\omega), C\left(\omega^{n}\right)\right) \leq n+\sqrt{(n-1)(n+3)}$.

(c) $\mathrm{d}\left(C(\omega), C\left(\omega^{n} k\right)\right) \leq n+1+\sqrt{n(n+4)}$.

Combinando o Corolário 5.5 e o Teorema 5.6 temos as funções $G(n, k)$ e $H(n, k)$ de (5.1) e (5.2). 


\subsection{Resultados preliminares}

Apresentaremos nesta seção dois resultados que terão um importante papel na demonstração do Teorema 5.4.

Proposição 5.7. Sejam L um espaço compacto de Hausdorff infinito com $L^{(2)}=\varnothing, K$ um espaço compacto de Hausdorff e $T$ um isomorfismo sobrejetor de $C(K)$ em $C(L)$ tal que $\left\|T^{-1}\right\|=1$. Suponha $1<n<\omega, x_{0} \in K^{(n)}, K_{0}$ uma vizinhança compacta de $x_{0}, 0<\epsilon<1$ e $h_{0} \in C(K)$ tais que $0 \leq h_{0} \leq 1, h_{0}(x)=1$ para cada $x \in K_{0}$ e $\left|T h_{0}(y)\right|<\epsilon$ para cada $y \in L^{(1)}$. Nessas condições existem pontos $x_{1}, \ldots, x_{n-1} \in K$, subconjuntos compactos $K_{1}, \ldots, K_{n-1}$ de $K$ e funções $h_{1}, \ldots, h_{n-1}$ em $C(K)$ verificando:

(a) $x_{i} \in \stackrel{\circ}{K}_{i} \cap K^{(n-i)}$, para $0 \leq i \leq n-1$.

(b) $K_{i} \subset \stackrel{\circ}{K}_{i-1}$, para $1 \leq i \leq n-1$.

(c) $0 \leq h_{i} \leq 1, h_{i}(x)=1$ se $x \in K_{i}$ e $h_{i}(x)=0$ se $x \notin \stackrel{\circ}{K}_{i-1}$, para $1 \leq i \leq n-1$.

(d) Os conjuntos $G_{i}=\left\{y \in L:\left|T h_{i}(y)\right| \geq \epsilon\right\}, 0 \leq i \leq n-1$, são conjuntos não vazios de pontos isolados mutuamente disjuntos.

Demonstração. Em virtude de $\left\|T^{-1}\right\|=1$ e $0<\epsilon<1$, o conjunto $G_{0}$ é não vazio, mais ainda, é finito. Do contrário, temos $G_{0} \cap L^{(1)} \neq \varnothing$ e isso contradiz a hipótese de $\left|T h_{0}(y)\right|<\epsilon$ para todo $y \in L^{(1)}$.

Dado $0 \leq r<n-1$, suponha que foram obtidos pontos $x_{0}, x_{1} \ldots, x_{r}$, subconjuntos compactos $K_{0}, K_{1} \ldots, K_{r}$ e funções $h_{0}, h_{1} \ldots, h_{r}$ em $C(K)$ satisfazendo os itens (a), (b), (c) e (d) acima.

Por $K$ ser um espaço topológico compacto de Hausdorff, é possível encontrar pontos $b_{1}, b_{2}, b_{3} \ldots$ em $\left(\stackrel{\circ}{K}_{r} \backslash\left\{x_{r}\right\}\right) \cap K^{(n-r-1)}$, subconjuntos abertos mutuamente disjuntos $U_{1}, U_{2}, U_{3} \ldots$ e compactos $M_{1}, M_{2}, M_{3} \ldots$ verificando

$$
b_{i} \in \stackrel{\circ}{M}_{i} \subset M_{i} \subset U_{i} \subset K_{r}, i \in \mathbb{N} .
$$

Aplicando o Lema de Urysohn 1.12 podemos obter funções $g_{1}, g_{2}, g_{3}, \ldots \in C(K)$ verificando para cada $i \in \mathbb{N}, 0 \leq g_{i}(x) \leq 1$ para todo $x \in K, g_{i}(x)=1$ se $x \in M_{i}$ e $g_{i}(x)=0$ se $x \notin U_{i}$. Em virtude de $U_{i} \cap U_{j}=\varnothing$ se $i \neq j$, temos $g_{i} \cdot g_{j}=0$ se $i \neq j$. Novamente por $\left\|T^{-1}\right\|=1$ e $0<\epsilon<1$ deduzimos que $\left\{y \in L:\left|T g_{i}(y)\right| \geq \epsilon\right\} \neq \varnothing$ para todo $i \in \mathbb{N}$.

Seja $G=G_{1} \cup \ldots \cup G_{r}$. Afirmamos que existe $s \in \mathbb{N}$ tal que

$$
\left\{y \in L:\left|T g_{s}(y)\right| \geq \epsilon\right\} \cap\left(G \cup L^{(1)}\right)=\varnothing .
$$

Do contrário, assumindo $G \cup L^{(1)}=\left\{y_{1}, \ldots, y_{t}\right\}$ e denotando

$$
\Gamma_{i}=\left\{j \in \mathbb{N}:\left|T g_{j}\left(y_{i}\right)\right| \geq \epsilon\right\}, 1 \leq i \leq t,
$$

temos

$$
\mathbb{N} \subseteq \Gamma_{1} \cup \ldots \cup \Gamma_{t}
$$

e portanto $\Gamma_{p}$ é infinito para algum $1 \leq p \leq t$. Sejam $p_{1}, p_{2}, p_{3} \ldots$ inteiros distintos em $\Gamma_{p}$.

Seja $m \in \mathbb{N}$ tal que $\epsilon \cdot m>\|T\|$ e para cada $1 \leq i \leq m$ seja $r_{i}$ um escalar satisfazendo

$$
r_{i} \cdot T g_{p_{i}}\left(y_{p}\right)=\left|T g_{p_{i}}\left(y_{p}\right)\right| .
$$


Em virtude de $g_{i} \cdot g_{j}=0$ se $i \neq j$, a função $g=\sum_{i=1}^{m} r_{i} \cdot g_{p_{i}} \in C(K)$ verifica $\|g\| \leq 1$. Entretanto,

$$
\begin{aligned}
\|T\| \geq\|T g\| & \geq\left|T\left(\sum_{i=1}^{m} r_{i} \cdot g_{p_{i}}\right)\left(y_{p}\right)\right| \\
& =\left|\sum_{i=1}^{m} r_{i} \cdot T g_{p_{i}}\left(y_{p}\right)\right|=\sum_{i=1}^{m}\left|T g_{p_{i}}\left(y_{p}\right)\right|>\|T\|,
\end{aligned}
$$

uma contradição que estabelece nossa afirmação.

Finalmente, podemos fixar $s \in \mathbb{N}$ verificando (5.3) e definir $x_{r+1}=b_{s}, K_{r+1}=M_{s}, h_{r+1}=g_{s}$ e $G_{r+1}=\left\{y \in L:\left|T g_{s}(y)\right| \geq \epsilon\right\}$. É fácil verificar que as condições (a), (b), (c) e (d) estão satisfeitas para $r+1$. Isso completa a demonstração.

Lema 5.8. Sejam L um conjunto compacto de Hausdorff infinito com $L^{(2)}=\varnothing, K$ um espaço compacto de Hausdorff e $T$ um isomorfismo de $C(K)$ em $C(L)$. Suponha que $\left|K^{(n)}\right|>\left|L^{(1)}\right|$ para algum $1 \leq n<\omega$. Então, para cada $\epsilon>0$ existe $x_{0} \in K^{(n)}$, uma vizinhança compacta $K_{0}$ de $x_{0}$ e uma função $h \in C(K)$ tal que $0 \leq h(x) \leq 1$ para todo $x \in K, h(x)=1$ se $x \in K_{0}$ e tal que $|T h(y)|<\epsilon$ se $y \in L^{(1)}$.

Demonstração. Por contradição, suponha $\epsilon>0$ tal que $|T h(y)| \geq \epsilon$ para algum $y \in L^{(1)}$ sempre que $h \in C(K)$ for tal que $h(x)=1$ para todo $x$ em um subconjunto fechado $K_{0}$ satisfazendo $\stackrel{\circ}{K}_{0} \cap K^{(n)} \neq \varnothing$.

Suponha $\left|L^{(1)}\right|=m$ e fixe $x_{1}, \ldots, x_{m+1}$ pontos distintos em $K^{(n)}$ com respectivas vizinhanças compactas disjuntas $A_{1}, \ldots, A_{m+1}$. Utilizando o Lema de Urysohn 1.12 é possivel encontrar funções $h_{i} \in C(K), 1 \leq i \leq m+1$, tais que $0 \leq h_{i}(x) \leq 1$ para todo $x \in K, h_{i}(x)=1$ se $x \in A_{i}$ e satisfazendo $h_{i} \cdot h_{j}=0$ se $i \neq j$.

Seja $l_{\infty}^{m+1}$ o espaço $\mathbb{K}^{m+1}$ munido da norma do máximo. Para cada $a=\left(a_{1}, \ldots, a_{m+1}\right) \in l_{\infty}^{m+1}$ associamos a função

$$
\gamma_{a}=\sum_{i=1}^{m+1} a_{i} \cdot h_{i} \in C(K) .
$$

Observe que $\left\|\gamma_{a}\right\|=\|a\|, a \in l_{\infty}^{m+1}$. Identificando, de maneira natural, o espaço $C\left(L^{(1)}\right)$ com $l_{\infty}^{m}$, considere $S: l_{\infty}^{m+1} \rightarrow l_{\infty}^{m}$, a aplicação definida por

$$
S(a)=\left.T \gamma_{a}\right|_{L^{(1)}}, a \in l_{\infty}^{m+1} .
$$

Claramente $S$ é um operador linear. De nossa suposição inicial deduzimos que para cada $a \in l_{\infty}^{m+1}$ existe $y \in L^{(1)}$ verificando

$$
\|S(a)\|=\left|T \gamma_{a}(y)\right| \geq \epsilon\|a\| .
$$

Portanto, $S$ é um isomorfismo de $l_{\infty}^{m+1}$ em $l_{\infty}^{m}$, o que é uma contradição.

\subsection{Cotas inferiores para as distâncias entre $C(K)$ e $C(L), L^{(2)}=\varnothing$}

Utilizando os resultados da Seção 5.2, vamos estabelecer o Teorema 5.4.

Demonstração do Teorema 5.4. Vamos assumir a existência de um isomorfismo sobrejetor $T$ de $C(K)$ em $C(L)$ tal que $\|T\|\left\|T^{-1}\right\|<2 n+1$ e obter uma contradição.

Sem perda de generalidade vamos assumir que $\left\|T^{-1}\right\|=1$, pois do contrário podemos simplesmente substituir $T$ pelo isomorfismo $\left\|T^{-1}\right\| T$ que claramente possui estas propriedades. 
Sejam $0<\eta, \epsilon<1$ verificando

$$
\|T\|<(2 n+1) \frac{1-\epsilon}{1+\epsilon} \quad \text { e } \quad \eta<\min \left\{\epsilon, \frac{(2 n+1)(1-\epsilon)-\|T\|}{2}\right\} .
$$

De acordo com o Lema 5.8 existe $x_{0} \in K^{(n)}$, uma vizinhança compacta $K_{0}$ de $x_{0}$ e uma função $h_{0} \in C(K)$ tal que $0 \leq h_{0}(x) \leq 1$ para todo $x \in K, h_{0}(x)=1$ se $x \in K_{0}$ e tal que $\left|T h_{0}(y)\right|<\epsilon$ se $y \in L^{(1)}$. Relacionados a $x_{0}, K_{0}, h_{0}$ e $\epsilon>0$, considere pontos $x_{1}, \ldots, x_{n-1} \in K$, subconjuntos compactos $K_{1}, \ldots, K_{n-1} \subset K$, funções $h_{1}, \ldots, h_{n-1} \in C(K)$ e subconjuntos $G_{0}, G_{1} \ldots, G_{n-1} \subset L$ verificando os itens (a), (b), (c) e (d) da Proposição 5.7.

Defina

$$
g_{i}=\mathbb{1}_{G_{i}} \cdot T h_{i}, 0 \leq i \leq n-1,
$$

onde $\mathbb{1}_{G_{i}}$ denota a função característica de $G_{i}$. Observe que $g_{i} \in C(L)$ para cada $0 \leq i \leq n-1$.

Seja $G$ o conjunto finito $\bigcup_{i=0}^{n-1} G_{i}$ e para cada $y \in G$ seja $\delta_{y}$ a medida de Dirac concentrada em $y$. Utilizando o Teorema 1.17 deduzimos que

$$
H=\bigcup_{y \in G}\left\{x \in K:\left|T^{*}\left(\delta_{y}\right)\right|(\{x\})>\eta\right\}
$$

é um conjunto finito. Existe, portanto, um ponto $z \in \stackrel{\circ}{K}_{n-1} \backslash H$ tal que $\left|T^{*}\left(\delta_{y}\right)\right|(\{z\})<\eta$ para todo $y \in G$. Devido a regularidade das medidas, podemos encontrar uma vizinhança aberta de $z$, $U \subset K_{n-1}$, satisfazendo

$$
\left|T^{*}\left(\delta_{y}\right)\right|(U)<\eta, y \in G .
$$

Aplicando o Lema de Urysohn 1.12, podemos fixar uma função $h_{n} \in C(K)$, tal que $0 \leq h_{n}(x) \leq 1$ para todo $x \in K, h_{n}(z)=1$ e $h_{n}(x)=0$ se $x \notin U$. Seja $\alpha \in C(L)$ a função definida por

$$
\alpha(y)=g_{0}(y)+2 \sum_{i=1}^{n-1} g_{i}(y)+2 T h_{n}(y), y \in L .
$$

Passo 1. Vamos demonstrar que $\|\alpha\|=\max \left\{2\left\|T h_{n}\right\|,|\alpha(y)|: y \in G\right\}$.

Para estabelecer este resultado observe que para cada $y \in G$ vale

$$
\left|T h_{n}(y)\right|=\left|\int T h_{n} d \delta_{y}\right|=\left|\int h_{n} d T^{*}\left(\delta_{y}\right)\right| \leq\left|T^{*}\left(\delta_{y}\right)\right|(U)<\eta<1 .
$$

Por outro lado, se $y \mapsto\left|T h_{n}(y)\right|$ atinge seu máximo em $y_{0} \in L$, temos, em virtude de $\left\|T^{-1}\right\|=1$,

$$
\left|T h_{n}\left(y_{0}\right)\right|=\left\|T h_{n}\right\| \geq 1
$$

e consequentemente $y_{0} \in L \backslash G$. O Passo 2 fica estabelecido notando-se que $\alpha(y)=2 T h_{n}(y)$ para todo $y \in L \backslash G$.

Passo 2. Vamos demonstrar que $\|\alpha\| \geq(2 n+1)-(2 n-1) \epsilon$. 
Em virtude de $\left\|T^{-1}\right\|=1$

$$
\begin{aligned}
\|\alpha\| & =\left\|g_{0}+2 \sum_{i=1}^{n-1} g_{i}+2 T h_{n}\right\| \geq\left\|T^{-1} g_{0}+2 \sum_{i=1}^{n-1} T^{-1} g_{i}+2 h_{n}\right\| \\
& \geq\left|\left(h_{0}(z)+2 \sum_{i=1}^{n} h_{i}(z)\right)-\left(h_{0}(z)-T^{-1} g_{0}(z)\right)-2 \sum_{i=1}^{n-1}\left(h_{i}(z)-T^{-1} g_{i}(z)\right)\right| \\
& \geq\left|h_{0}(z)+2 \sum_{i=1}^{n} h_{i}(z)\right|-\left|h_{0}(z)-T^{-1} g_{0}(z)\right|-2 \sum_{i=1}^{n-1}\left|h_{i}(z)-T^{-1} g_{i}(z)\right| .
\end{aligned}
$$

Como $\|f\| \leq\|T f\|, f \in C(K)$, temos

$\left|h_{i}(z)-T^{-1} g_{i}(z)\right| \leq\left\|h_{i}-T^{-1} g_{i}\right\| \leq\left\|T h_{i}-g_{i}\right\|=\left\|\left(1-\mathbb{1}_{G_{i}}\right) \cdot T h_{i}\right\| \leq \epsilon, 0 \leq i \leq n-1$.

Combinando (5.6), (5.7) e recordando a definição das funções $h_{i}$, deduzimos

$$
\|\alpha\| \geq(2 n+1)-(2 n-1) \epsilon .
$$

Passo 3. Em virtude de $\|\alpha\| \geq(2 n+1)-(2 n-1) \epsilon$, de acordo com o Passo 1, temos duas possibilidades:

(i) $2\left\|T h_{n}\right\| \geq(2 n+1)-(2 n-1) \epsilon$,

(ii) $|\alpha(y)| \geq(2 n+1)-(2 n-1) \epsilon$ para algum $y \in G$.

Vamos demonstrar que ambas as possibilidades implicam uma contradição.

Suponha inicialmente que (i) seja verdadeira. Fixe

$$
A=T^{-1} g_{0}-2 h_{n} .
$$

Recordando que $0 \leq h_{n} \leq h_{0} \leq 1$ e $\|f\| \leq\|T f\|, f \in C(K)$, temos, para cada $x \in K$,

$$
\begin{aligned}
\left|T^{-1} g_{0}(x)-2 h_{n}(x)\right| & \leq\left|h_{0}(x)-2 h_{n}(x)\right|+\left|T^{-1} g_{0}(x)-h_{0}(x)\right| \\
& \leq 1+\left\|T^{-1} g_{0}-h_{0}\right\| \leq 1+\left\|g_{0}-T h_{0}\right\| \leq 1+\epsilon .
\end{aligned}
$$

Portanto $\|A\| \leq 1+\epsilon$.

Devido a (5.4) e (5.5) existe $y_{0} \in L \backslash G$ tal que $\left\|T h_{n}\right\|=\left|T h_{n}\left(y_{0}\right)\right|$. Podemos escrever

$$
\left|T(A)\left(y_{0}\right)\right|=2\left|T h_{n}\left(y_{0}\right)\right|=2\left\|T h_{n}\right\| \geq(2 n+1)-(2 n-1) \epsilon>(2 n+1)(1-\epsilon) .
$$

Consequentemente

$$
\|T\| \geq\left\|T\left(\frac{1}{1+\epsilon} A\right)\right\|>(2 n+1) \frac{1-\epsilon}{1+\epsilon} .
$$

Isto contradiz a escolha de $\epsilon$.

Em seguida, assuma que (ii) seja verdadeiro. Distinguimos dois casos.

Caso 1. $\|\alpha\|=\left|\alpha\left(y_{0}\right)\right|$ para algum $y_{0} \in G_{0}$. Neste caso, por $G_{0}, G_{1} \ldots, G_{n-1}$ serem mutuamente disjuntos, temos

$$
\left|\alpha\left(y_{0}\right)\right|=\left|g_{0}\left(y_{0}\right)+2 T h_{n}\left(y_{0}\right)\right| \geq(2 n+1)-(2 n-1) \epsilon .
$$


Pela escolha de $\eta$,

$$
\begin{aligned}
\left|g_{0}\left(y_{0}\right)\right| & \geq(2 n+1)-(2 n-1) \epsilon-2\left|T h_{n}\left(y_{0}\right)\right| \\
& >(2 n+1)-(2 n-1) \epsilon-2 \eta>\|T\| .
\end{aligned}
$$

Consequentemente

$$
\|T\| \geq\left\|T h_{0}\right\| \geq\left|T h_{0}\left(y_{0}\right)\right|=\left|g_{0}\left(y_{0}\right)\right|>\|T\|,
$$

o que é uma contradição.

Caso 2. $\|\alpha\|=\left|\alpha\left(y_{0}\right)\right|$ para algum $y_{0} \in G_{i}, i>0$. Por $G_{0}, G_{1} \ldots, G_{n-1}$ serem mutuamente disjuntos, temos

$$
\left|\alpha\left(y_{0}\right)\right|=\left|2 g_{i}\left(y_{0}\right)+2 T h_{n}\left(y_{0}\right)\right| \geq(2 n+1)-(2 n-1) \epsilon .
$$

Por (5.4) e por $\eta<\epsilon$,

$$
2\left|g_{i}\left(y_{0}\right)\right| \geq(2 n+1)-(2 n-1) \epsilon-2\left|T h_{n}\left(y_{0}\right)\right|>(2 n+1)(1-\epsilon) .
$$

Considere a função

$$
B_{i}=T^{-1} g_{0}-2 h_{i}
$$

Recordando que $0 \leq h_{i} \leq h_{0} \leq 1$ e $\|f\| \leq\|T f\|, f \in C(K)$, temos, para cada $x \in K$,

$$
\begin{aligned}
\left|T^{-1} g_{0}(x)-2 h_{i}(x)\right| & \leq\left|h_{0}(x)-2 h_{i}(x)\right|+\left|T^{-1} g_{0}(x)-h_{0}(x)\right| \\
& \leq 1+\left\|T^{-1} g_{0}-h_{0}\right\| \leq 1+\left\|g_{0}-T h_{0}\right\| \leq 1+\epsilon .
\end{aligned}
$$

Resulta que $\left\|B_{i}\right\| \leq 1+\epsilon$. Mais ainda, da relação (5.8) decorre

$$
\left|T\left(B_{i}\right)\left(y_{0}\right)\right|=2\left|T h_{i}\left(y_{0}\right)\right|=2\left|g_{i}\left(y_{0}\right)\right|>(2 n+1)(1-\epsilon) .
$$

Consequentemente,

$$
\|T\| \geq\left\|T\left(\frac{1}{1+\epsilon} B_{i}\right)\right\|>(2 n+1) \frac{1-\epsilon}{1+\epsilon},
$$

o que contradiz a escolha de $\epsilon$.

\subsection{Cotas superiores para as distâncias entre $C(\omega)$ e $C\left(\omega^{n} k\right), 1 \leq$} $n, k<\omega$

O objetivo desta seção é demonstrar o Teorema 5.6. Recordando a Definição 4.9 do capítulo anterior, precisamos do seguinte lema fundamental.

Lema 5.9. Sejam ordinais $1 \leq n, k<\omega$ e números reais $A, B, C, D, E$. Para cada $f \in C\left(\omega^{n} k\right)$ considere a sequência $\left(a_{\xi}\right)_{1 \leq \xi \leq \omega^{n} k}$,

$$
a_{\xi}= \begin{cases}A & \text { se } \xi=\omega^{n} k, \\ B\left(f(\xi)-f\left(\xi^{[1]}\right)\right) & \text { se } \xi=\omega^{n}(k-1)+\omega^{n-1} i, 1 \leq i<\omega, \\ C\left(f(\xi)-f\left(\xi^{[1]}\right)\right) & \text { se } \xi \in \omega^{n}(k-1)+\omega^{n-1}(i-1), \omega^{n}(k-1)+\omega^{n-1} i[, 1 \leq i<\omega \\ D\left(f\left(\omega^{n} r\right)-f\left(\omega^{n} k\right)\right) & \text { se } \xi=\omega^{n} r, 1 \leq r \leq k-1, \\ E\left(f(\xi)-f\left(\xi^{[1]}\right)\right) & \text { se } \xi \in] \omega^{n}(r-1), \omega^{n} r[, 1 \leq r \leq k-1 .\end{cases}
$$


Então para cada $\epsilon>0$ existem somente um número finito de ordinais $1 \leq \xi \leq \omega^{n} k$ satisfazendo $\left|a_{\xi}\right| \geq \epsilon$.

Demonstração. Sejam $f \in C\left(\omega^{n} k\right)$ e $\epsilon>0$ arbitrários e $\left(a_{\xi}\right)_{1 \leq \xi \leq \omega^{n} k}$ como acima. Considere a função $g \in C\left(\omega^{n}\right)$,

$$
g(\xi)=f\left(\omega^{n}(k-1)+\xi\right), 1 \leq \xi \leq \omega^{n},
$$

e a sequência $\left(b_{\xi}\right)_{1 \leq \xi \leq \omega^{n}}$ definida por

$$
b_{\xi}= \begin{cases}A & \text { se } \xi=\omega^{n} \\ B\left(g(\xi)-g\left(\xi^{[1]}\right)\right) & \text { se } \xi=\omega^{n-1} i, 1 \leq i<\omega, \\ C\left(g(\xi)-g\left(\xi^{[1]}\right)\right) & \text { se } \xi \in] \omega^{n-1}(i-1), \omega^{n-1} i[, 1 \leq i<\omega .\end{cases}
$$

Por consequência do Lema 4.11, existe somente um número finito de ordinais $1 \leq \xi \leq \omega^{n}$ satisfazendo $\left|b_{\xi}\right| \geq \epsilon$. Em virtude de

$$
b_{\xi}=a_{\omega^{n}(k-1)+\xi}, 1 \leq \xi \leq \omega^{n},
$$

deduzimos que existe somente um número finito de ordinais $\xi$ no intervalo $\left[\omega^{n}(k-1)+1, \omega^{n} k\right]$ satisfazendo $\left|a_{\xi}\right| \geq \epsilon$. Se $k>1$, para cada $1 \leq r \leq k-1$ defina $h_{r} \in C\left(\omega^{n}\right)$ como

$$
h_{r}(\xi)=f\left(\omega^{n}(r-1)+\xi\right), 1 \leq \xi \leq \omega^{n} .
$$

Em seguida fixe a sequência $\left(c_{\xi}^{r}\right)_{1 \leq \xi \leq \omega^{n}}$,

$$
c_{\xi}^{r}= \begin{cases}D\left(f\left(\omega^{n} r\right)-f\left(\omega^{n} k\right)\right) & \text { se } \xi=\omega^{n}, \\ E\left(h_{r}(\xi)-h_{r}\left(\xi^{[1]}\right)\right) & \text { se } 1 \leq \xi<\omega^{n} .\end{cases}
$$

Mais uma vez pelo Lema 4.11, para cada $1 \leq r \leq k-1$, existe somente um número finito de ordinais $1 \leq \xi \leq \omega^{n}$ satisfazendo $\left|c_{\xi}^{r}\right| \geq \epsilon$. Em virtude de

$$
c_{\xi}^{r}=a_{\omega^{n}(r-1)+\xi}, 1 \leq \xi \leq \omega^{n},
$$

podemos concluir que para cada $1 \leq r \leq k-1$, existe somente um número finito de ordinais $\xi$ no intervalo $\left[\omega^{n}(r-1)+1, \omega^{n} r\right]$ satisfazendo $\left|a_{\xi}\right| \geq \epsilon$. Como $\left[1, \omega^{n} k\right]$ é a união dos intervalos $\left[1, \omega^{n}\right], \ldots,\left[\omega^{n}(k-2)+1, \omega^{n}(k-1)\right]$ e $\left[\omega^{n}(k-1)+1, \omega^{n} k\right]$, o lema está demonstrado.

Estamos agora em condições de demonstrar o Teorema 5.6.

Demonstração do Teorema 5.6. Sejam $\Gamma_{n, k}, 1 \leq k, n<\omega$, o intervalo de ordinal $] 0, \omega^{n} k[$ munido da topologia discreta, $K_{n, k}=\Gamma_{n, k} \dot{\cup}\left\{\omega^{n} k\right\}$ o compactificado de Aleksandrov de $\Gamma_{n, k}$. Para simplificar a demonstração vamos substituir o espaço $C(\omega)$ por $C\left(K_{n, k}\right)$. Estes espaços são claramente isometricamente isomorfos.

Seja $A>1$. Para cada $f \in C\left(\omega^{n} k\right)$ defina a função $T(f): K_{n, k} \rightarrow \mathbb{K}$ por

$$
T(f)(\xi)= \begin{cases}f\left(\omega^{n} k\right) & \text { se } \xi=\omega^{n} k, \\ A f(\xi)-(A-1) f\left(\xi^{[1]}\right) & \text { se } \xi=\omega^{n}(k-1)+\omega^{n-1} i, 1 \leq i<\omega, \\ \frac{(n-1) A}{A-1}\left(f(\xi)-f\left(\xi^{[1]}\right)\right)+f\left(\omega^{n} k\right) & \text { se } \xi \in] \omega^{n}(k-1)+\omega^{n-1}(i-1), \omega^{n}(k-1)+\omega^{n-1} i[, \\ & 1 \leq i<\omega, \\ A f\left(\omega^{n} r\right)-(A-1) f\left(\omega^{n} k\right) & \text { se } \xi=\omega^{n} r, 1 \leq r \leq k-1, \\ \frac{n A}{A-1}\left(f(\xi)-f\left(\xi^{[1]}\right)\right)+f\left(\omega^{n} k\right) & \text { se } \xi \in] \omega^{n}(r-1), \omega^{n} r[, 1 \leq r \leq k-1 .\end{cases}
$$


Vamos verificar que $T(f) \in C\left(K_{n, k}\right), f \in C\left(\omega^{n} k\right)$. De fato, dado $f \in C\left(\omega^{n} k\right)$ considere a função

$$
F=T(f)-f\left(\omega^{n} k\right) .
$$

De forma mais explícita

$$
F(\xi)= \begin{cases}0 & \text { se } \xi=\omega^{n} k, \\ A\left(f(\xi)-f\left(\xi^{[1]}\right)\right) & \text { se } \xi=\omega^{n}(k-1)+\omega^{n-1} i, 1 \leq i<\omega, \\ \frac{(n-1) A}{A-1}\left(f(\xi)-f\left(\xi^{[1]}\right)\right) & \text { se } \xi \in] \omega^{n}(k-1)+\omega^{n-1}(i-1), \omega^{n}(k-1)+\omega^{n-1} i[, 1 \leq i<\omega, \\ A\left(f\left(\omega^{n} r\right)-f\left(\omega^{n} k\right)\right) & \text { se } \xi=\omega^{n} r, 1 \leq r \leq k-1, \\ \frac{n A}{A-1}\left(f(\xi)-f\left(\xi^{[1]}\right)\right) & \text { se } \xi \in] \omega^{n}(r-1), \omega^{n} r[, 1 \leq r \leq k-1 .\end{cases}
$$

De acordo com o Lema 5.9, para cada $\epsilon>0$ existe somente um número de ordinais $\xi$ no intervalo $\left[1, \omega^{n} k\right]$ satisfazendo $|F(\xi)| \geq \epsilon$. Resulta que $T(f)$ é contínua em $\omega^{n} k, \operatorname{logo}, T(f) \in C\left(K_{n, k}\right)$.

De maneira similar a (4.14) e (4.15) no capítulo anterior, demonstra-se que $T$ define um operador linear de $C\left(\omega^{n} k\right)$ em $C\left(K_{n, k}\right)$. Mais ainda, se $k>1$, então $T$ verifica

$$
\|T\| \leq \max \left\{\frac{2 n A}{A-1}+1,2 A-1\right\} .
$$

Se $k=1$ e $n>1$, então

$$
\|T\| \leq \max \left\{\frac{2(n-1) A}{A-1}+1,2 A-1\right\} .
$$

Em seguida, recordando a Observação 4.10, vamos utilizar o fato de que cada número ordinal $1 \leq \xi<\omega^{n+1}$ admite uma única representação na forma

$$
\xi=\omega^{n} i_{0}+\omega^{n-1} i_{1}+\ldots+\omega^{n-(j-1)} i_{j-1}+\omega^{n-j} i_{j}
$$

onde $0 \leq j \leq n, 1 \leq i_{j}<\omega$ e $0 \leq i_{r}<\omega$ se $0 \leq r \leq j-1$.

Para cada função $g \in C\left(K_{n, k}\right)$ defina a aplicação $S(g):\left[1, \omega^{n} k\right] \rightarrow \mathbb{K}$ por

$$
S(g)(\xi)= \begin{cases}g\left(\omega^{n} k\right) & \text { se } \xi=\omega^{n} k, \\ \frac{1}{A} g(\xi)+\frac{A-1}{A} g\left(\omega^{n} k\right) & \text { se } \xi=\omega^{n}(k-1)+\omega^{n-1} i, 1 \leq i<\omega, \\ \frac{1}{A} g\left(\omega^{n} r\right)+\frac{A-1}{A} g\left(\omega^{n} k\right) & \text { se } \xi=\omega^{n} r, 1 \leq r \leq k-1 .\end{cases}
$$

Se $\xi \in] \omega^{n}(k-1)+\omega^{n-1}(i-1), \omega^{n}(k-1)+\omega^{n-1} i[, 1 \leq i<\omega$, e $\xi$ está representado como (5.11), então

$$
S(g)(\xi)=\frac{A-1}{A(n-1)} \sum_{s=0}^{j-2}\left(g\left(\xi^{[s]}\right)-g\left(\omega^{n} k\right)\right)+\frac{1}{A} g\left(\xi^{[j-1]}\right)+\frac{A-1}{A} g\left(\omega^{n} k\right),
$$

e se $\xi \in] \omega^{n}(r-1), \omega^{n} r[, 1 \leq r \leq k-1$, e $\xi$ está representado como (5.11), então

$$
S(g)(\xi)=\frac{A-1}{A n} \sum_{s=0}^{j-1}\left(g\left(\xi^{[s]}\right)-g\left(\omega^{n} k\right)\right)+\frac{1}{A} g\left(\xi^{[j]}\right)+\frac{A-1}{A} g\left(\omega^{n} k\right) .
$$

Vamos demonstrar que $S(g)$ é contínua em $\left[1, \omega^{n} k\right]$ para cada $g \in C\left(K_{n, k}\right)$. Para tanto, seja $g \in C\left(K_{n, k}\right)$ arbitrário. Dado $\xi_{0}$ um ponto não isolado de $\left[1, \omega^{n} k\right]$ e $\epsilon>0$ defina

$$
\Lambda_{\epsilon}=\left\{1 \leq \xi \leq \omega^{n} k:\left|g(\xi)-g\left(\omega^{n} k\right)\right| \geq \frac{\epsilon}{n}\right\} .
$$


Para benefício do leitor distinguimos dois casos.

Caso 1. $\xi_{0}=\omega^{n} k$. Por $\Lambda_{\epsilon}$ ser um conjunto finito existe $1 \leq m<\omega$ tal que

$$
] \omega^{n}(k-1)+\omega^{n-1} m, \omega^{n} k\left[\cap \Lambda_{\epsilon}=\varnothing .\right.
$$

Segue da definição de $S(g)$ que se $\xi \in] \omega^{n}(k-1)+\omega^{n-1} m, \omega^{n} k[$, então

$$
\left|S(g)(\xi)-S(g)\left(\xi_{0}\right)\right| \leq\left|g\left(\xi_{1}\right)-g\left(\omega^{n} k\right)\right|+\ldots+\left|g\left(\xi_{s}\right)-g\left(\omega^{n} k\right)\right|,
$$

onde $1 \leq s \leq n$ e $\xi=\xi_{1}<\ldots<\xi_{s}<\omega^{n} k$. Portanto

$$
\left|S(g)(\xi)-S(g)\left(\xi_{0}\right)\right|<\epsilon
$$

Caso 2. $1 \leq \xi_{0}<\omega^{n} k$. Escreva $\xi_{0}=\omega^{n} i_{0}+\omega^{n-1} i_{1}+\ldots+\omega^{n-j} i_{j}, 0 \leq j<n$, onde $0 \leq i_{0} \leq k-1$, $1 \leq i_{j}<\omega$ e $0 \leq i_{r}<\omega$ se $1 \leq r \leq j-1$. Por $\Lambda_{\epsilon}$ ser finito existe $1 \leq m<\omega$ tal que

$$
] \omega^{n} i_{0}+\omega^{n-1} i_{1}+\ldots+\omega^{n-j}\left(i_{j}-1\right)+\omega^{n-(j+1)} m, \omega^{n} i_{0}+\omega^{n-1} i_{1}+\ldots+\omega^{n-j} i_{j}\left[\cap \Lambda_{\epsilon}=\varnothing .\right.
$$

Observe que se

$$
\xi \in] \omega^{n} i_{0}+\omega^{n-1} i_{1}+\ldots+\omega^{n-j}\left(i_{j}-1\right)+\omega^{n-(j+1)} m, \omega^{n} i_{0}+\omega^{n-1} i_{1}+\ldots+\omega^{n-j} i_{j}[,
$$

então existe $1 \leq s \leq n-j$ tal que $\xi^{[s]}=\xi_{0}$. Temos, pela definição de $S(g)$,

$$
\left|S(g)(\xi)-S(g)\left(\xi_{0}\right)\right| \leq\left|g\left(\xi_{1}\right)-g\left(\omega^{n} k\right)\right|+\ldots+\left|g\left(\xi_{s}\right)-g\left(\omega^{n} k\right)\right|
$$

onde $\xi=\xi_{1}<\ldots<\xi_{s}<\xi_{0}$. Consequentemente

$$
\left|S(g)(\xi)-S(g)\left(\xi_{0}\right)\right|<\epsilon .
$$

Deduzimos que $S(g)$ é contínua em $\xi_{0}$.

Portanto, $S$ define uma aplicação de $C\left(K_{n, k}\right)$ em $C\left(\omega^{n} k\right)$. Vamos verificar que as aplicações $S \circ T$ e $T \circ S$ são, respectivamente, os operadores identidade em $C\left(\omega^{n} k\right)$ e $C\left(K_{n, k}\right)$. Com efeito, seja $f \in C\left(\omega^{n} k\right)$ arbitrário. Se $\xi=\omega^{n} k$, então

$$
S \circ T(f)\left(\omega^{n} k\right)=T(f)\left(\omega^{n} k\right)=f\left(\omega^{n} k\right) .
$$

Se $\xi=\omega^{n}(k-1)+\omega^{n-1} i, 1 \leq i<\omega$, então

$$
\begin{aligned}
S \circ T(f)(\xi) & =\frac{1}{A} T(f)(\xi)+\frac{A-1}{A} T(f)\left(\omega^{n} k\right) \\
& =\frac{1}{A}\left(A f(\xi)-(A-1) f\left(\omega^{n} k\right)\right)+\frac{A-1}{A} f\left(\omega^{n} k\right)=f(\xi) .
\end{aligned}
$$

Se $\xi=\omega^{n} r, 1 \leq r \leq k-1$, então

$$
\begin{aligned}
S \circ T(f)(\xi) & =\frac{1}{A} T(f)\left(\omega^{n} r\right)+\frac{A-1}{A} T(f)\left(\omega^{n} k\right) \\
& =\frac{1}{A}\left(A f\left(\omega^{n} r\right)-(A-1) f\left(\omega^{n} k\right)\right)+\frac{A-1}{A} f\left(\omega^{n} k\right)=f(\xi) .
\end{aligned}
$$

Se $\xi \in] \omega^{n}(k-1)+\omega^{n-1}(i-1), \omega^{n}(k-1)+\omega^{n-1} i[, 1 \leq i<\omega$, e $\xi$ está representado como 
(5.11), então

$$
\begin{aligned}
S \circ T(f)(\xi) & =\frac{A-1}{A(n-1)} \sum_{s=0}^{j-2}\left(T(f)\left(\xi^{[s]}\right)-T(f)\left(\omega^{n} k\right)\right)+\frac{1}{A} T(f)\left(\xi^{[j-1]}\right)+\frac{A-1}{A} T(f)\left(\omega^{n} k\right) \\
& =\frac{A-1}{A(n-1)} \sum_{s=0}^{j-2}\left(\frac{(n-1) A}{A-1}\left(f\left(\xi^{[s]}\right)-f\left(\xi^{[s+1]}\right)\right)\right)+f\left(\xi^{[j-1]}\right) \\
& =\left(f(\xi)-f\left(\xi^{[j-1]}\right)\right)+f\left(\xi^{[j-1]}\right)=f(\xi) .
\end{aligned}
$$

Se $\xi \in] \omega^{n}(r-1), \omega^{n} r[, 1 \leq r \leq k-1$, e $\xi$ está representado como (5.11), então

$$
\begin{aligned}
S \circ T(f)(\xi) & =\frac{A-1}{A n} \sum_{s=0}^{j-1}\left(T(f)\left(\xi^{[s]}\right)-T(f)\left(\omega^{n} k\right)\right)+\frac{1}{A} T(f)\left(\xi^{[j]}\right)+\frac{A-1}{A} T(f)\left(\omega^{n} k\right) \\
& =\frac{A-1}{A n} \sum_{s=0}^{j-1}\left(\frac{n A}{A-1}\left(f\left(\xi^{[s]}\right)-f\left(\xi^{[s+1]}\right)\right)\right)+f\left(\xi^{[j]}\right) \\
& =\left(f(\xi)-f\left(\xi^{[j]}\right)\right)+f\left(\xi^{[j]}\right)=f(\xi) .
\end{aligned}
$$

Concluímos que $S \circ T(f)=f$ para todo $f \in C\left(\omega^{n} k\right)$.

Por outro lado, fixe $g \in C_{0}\left(K_{n, k}\right)$ arbitrário. Se $\xi=\omega^{n} k$, então

$$
T \circ S(g)\left(\omega^{n} k\right)=S(g)\left(\omega^{n} k\right)=g\left(\omega^{n} k\right) .
$$

Se $\xi=\omega^{n}(k-1)+\omega^{n-1} i, 1 \leq i<\omega$, então

$$
\begin{aligned}
T \circ S(g)(\xi) & =A S(g)(\xi)-(A-1) S(g)\left(\omega^{n} k\right) \\
& =A\left(\frac{1}{A} g(\xi)+\frac{A-1}{A} g\left(\omega^{n} k\right)\right)-(A-1) g\left(\omega^{n} k\right)=g(\xi) .
\end{aligned}
$$

Se $\xi=\omega^{n} r, 1 \leq r \leq k-1$, então

$$
\begin{aligned}
T \circ S(g)(\xi) & =A S(g)\left(\omega^{n} r\right)-(A-1) S(g)\left(\omega^{n} k\right) \\
& =A\left(\frac{1}{A} g\left(\omega^{n} r\right)+\frac{A-1}{A} g\left(\omega^{n} k\right)\right)-(A-1) g\left(\omega^{n} k\right)=g(\xi) .
\end{aligned}
$$

Se $\xi \in] \omega^{n}(k-1)+\omega^{n-1}(i-1), \omega^{n}(k-1)+\omega^{n-1} i[, 1 \leq i<\omega$, e $\xi$ está representado como (5.11), então

$$
\begin{aligned}
T \circ S(g)(\xi) & =\frac{(n-1) A}{A-1}\left(S(g)(\xi)-S(g)\left(\xi^{[1]}\right)\right)+S(g)\left(\omega^{n} k\right) \\
& =\frac{(n-1) A}{A-1}\left(\frac{A-1}{A(n-1)}\left(g(\xi)-g\left(\omega^{n} k\right)\right)\right)+g\left(\omega^{n} k\right)=g(\xi) .
\end{aligned}
$$

Se $\xi \in] \omega^{n}(r-1), \omega^{n} r[, 1 \leq r \leq k-1$, e $\xi$ está representado como (5.11), então

$$
\begin{aligned}
T \circ S(g)(\xi) & =\frac{n A}{A-1}\left(S(g)(\xi)-S(g)\left(\xi^{[1]}\right)\right)+S(g)\left(\omega^{n} k\right) \\
& =\frac{n A}{A-1}\left(\frac{A-1}{A n}\left(g(\xi)-g\left(\omega^{n} k\right)\right)\right)+g\left(\omega^{n} k\right)=g(\xi) .
\end{aligned}
$$

Fica, portanto, estabelecido que $T \circ S(g)=g$ para cada $g \in C\left(K_{n, k}\right)$ e $S \circ T(f)=f$ para cada $f \in C\left(\omega^{n} k\right)$. Concluímos que $S$ é o operador inverso de $T$, mais ainda, $S$ é linear e verifica

$$
\|S\| \leq 1
$$


Se $k>1$, as relações (5.9) e (5.12) nos permitem escrever

$$
\mathrm{d}\left(C(\omega), C\left(\omega^{n} k\right)\right) \leq \inf _{1<A} \max \left\{\frac{2 n A}{A-1}+1,2 A-1\right\} .
$$

Observe que para $A>1$ a função $f(A)=\frac{2 n A}{A-1}+1$ é estritamente decrescente enquanto que a função $g(A)=2 A-1$ é estritamente crescente. Assim, o ínfimo em (5.13) é atingido quando $f(A)=g(A)$ e isto acontece quando

$$
A=\frac{n+2+\sqrt{n(n+4)}}{2} .
$$

Portanto

$$
\mathrm{d}\left(C(\omega), C\left(\omega^{n} k\right)\right) \leq n+1+\sqrt{n(n+4)} .
$$

Se $k=1$ e $n>1$, as relações (5.10) e (5.12) implicam

$$
\mathrm{d}\left(C(\omega), C\left(\omega^{n}\right)\right) \leq \inf _{1<A} \max \left\{\frac{2(n-1) A}{A-1}+1,2 A-1\right\} .
$$

De modo similar ao caso anterior, para $A>1$ a função $f(A)=\frac{2(n-1) A}{A-1}+1$ é estritamente decrescente enquanto que a função $g(A)=2 A-1$ é estritamente crescente. O ínfimo em (5.14) é atingido quando $f(A)=g(A)$, ou seja, quando

$$
A=\frac{n+1+\sqrt{(n-1)(n+3)}}{2} .
$$

Consequentemente

$$
\mathrm{d}\left(C(\omega), C\left(\omega^{n}\right)\right) \leq n+\sqrt{(n-1)(n+3)} .
$$




\section{Referências Bibliográficas}

[1] P. Aiena, M. González, On inessential and improjective operators. Studia Math. 131 (1998), $271-287$.

[2] D. Alspach, Y. Benyamini, Primariness of spaces of continuous functions on ordinals. Israel J. Math. 27 (1977), 64-92.

[3] D. Amir, On isomorphisms of continuous function spaces. Israel J. Math. 3 (1965), 205-210.

[4] S. Banach, Théorie des opérations linéaires. Monografie Matematyczne, Warsaw, 1932.

[5] J. W. Baker, Dispersed images of topological spaces and uncomplemented subspaces of $C(X)$. Proc. Amer. Math. Soc. 41 (1973), 309-314.

[6] E. Behrends and M. Cambern, An isomorphic Banach-Stone theorem. Studia Math. 90 (1988), $15-26$.

[7] C. Bessaga, A. Pełczyński, Spaces of continuous functions IV, Studia Math. 19 (1960), 53-62.

[8] R. S. Burachik, A. N. Iusem, Set-Valued Mappings and Enlargements of Monotone Operators. Optimization and Its Applications, Volume 8, Springer, New York, (2008).

[9] M. Cambern, Isomorphisms of $C_{0}(Y)$ onto $C(X)$. Pacific J. Math. 35 (1970), 307-312.

[10] M. Cambern, Isomorphisms of $C_{0}(Y)$ with $Y$ discrete. Math. Ann. 188 (1970), 23-25.

[11] M. Cambern, Isomorphims of spaces of continuous vector-valued functions. Illinois J. Math. 20 (1976), 1-11.

[12] M. Cambern, Isomorphisms of spaces of norm-continuous functions. Pacific J. Math. 116 (1985), 243-254.

[13] M. Cambern, On isomorphisms with small bound. Proc. Amer. Math. Soc. 18 (1967), 10621066.

[14] M. Cambern, On mappings of sequence spaces. Studia Math. 30 (1968), 73-77.

[15] P. Cembranos and J. Mendoza, Banach Spaces of Vector-Valued Functions. Lecture Notes in Mathematics 1676, Springer-Verlag, Berlin, 1997.

[16] B. Cengiz, On topological isomorphisms of $C_{0}(X)$ and the cardinal number of $X$. Proc. Amer. Math. Soc. 72 (1978), 105-108.

[17] J. A. Clarkson, Uniformly convex spaces. Trans. Amer. Math. Soc. 42 (1936), 396-414.

[18] H. B. Cohen, A bound-two isomorphism between $C(X)$ Banach spaces. Proc. Amer. Math. Soc. 50 (1975), 215-217. 
[19] H. B. Cohen, C.-H. Chu, Topological conditions for bound-2 isomorphisms of $C(X)$. Studia Math. 113 (1995), 1-24.

[20] J. Diestel, J.J. Uhl, Jr, Vector Measures. Math. Surveys 15, Amer. Math. Soc., Providence, 1977.

[21] J. Diestel, H. Jarchow, A. Tonge. Absolutely Summing Operators. Cambridge Studies in Advanced Mathematics, 43. Cambridge University Press, Cambridge, 1995.

[22] N. Dinculeanu. Vector Integration and Stochastic Integration in Banach Spaces. Wiley Interscience, 2000.

[23] N. Dinculeanu, Vector Measures . Pergamon Press, Berlin, 1967.

[24] P. Enflo, Banach spaces which can be given an equivalent uniformly convex norm. Israel J. Math. 13 (1972), 281-288.

[25] R. Engelking, General Topology. Sigma Ser. Pure Math. 6, Heldermann, Berlin, 1989.

[26] M. Fabian, P. Habala, P. Hájek, V. Montesinos, V. Zizler Banach Space Theory. CMS Books in Mathematics, Springer, New York, 2010.

[27] V. Ferenczi, A uniformly convex hereditarily indecomposable Banach space. Israel J. Math. 102 (1997), 199-225.

[28] V. Ferenczi, Hereditarily finitely decomposable Banach spaces. Studia Math. 123 (1997), 135149

[29] E. M. Galego, On solutions to the Schroeder-Bernstein problem for Banach spaces. Arch. Math. (Basel) 79 (2002), 299-307.

[30] J. Gil de Lamadrid, Measures and tensors. Canad. J. Math. 18 (1966), 762-793.

[31] Y. Gordon, On the distance coefficient between isomorphic function spaces. Israel J. Math. 8 (1970), 391-397.

[32] W. Hensgen, A simple proof of Singer's representation theorem. Proc. Amer. Math. Soc. 124 (1996), 3211-3212.

[33] R. C. James, Uniformly non-square Banach spaces. Ann. Math. 80 (1964), 542-550.

[34] K. Jarosz, Small isomorphisms of C(X,E) spaces. Pacific J. Math. 138 (1989), 295-315.

[35] W. B. Johnson, J. Lindenstrauss, Basics concepts in the geometry of Banach spaces. em: Handbook of the geometry of Banach spaces, Vol. 1, North-Holland, Amsterdam, 2001, 1-84.

[36] J. Lindenstrauss, L. Tzafriri, Classical Banach spaces I: Sequence Spaces. Ergebnisse der Mathematik und ihrer Grenzgebiete, Vol. 92, Springer-Verlag, Berlin-New York, 1977.

[37] W. Marciszewski. On Banach spaces $C(K)$ isomorphic to $c_{0}(\Gamma)$. Studia Math. 156 (2002), 295-302.

[38] B. Maurey, Type, cotype and K-convexity. Handbook of the geometry of Banach spaces, Vol. 2, 1299-1332, North-Holland, Amsterdam, 2003.

[39] S. Mazurkiewicz, W. Sierpiński, Contribution à la topologie des ensembles dénombrables. Fund. Math. 1 (1920), 17-27. 
[40] L. Meziani, On the dual space $C_{0}^{*}(S, X)$. Acta Math. Univ. Comenian. (N.S.) 78 (2009), 153160.

[41] Oja, È. F. Complemented spaces that are isomorphic to $l_{p}$ spaces in tensor products and operator spaces. (Russian) Sibirsk. Mat. Zh. 33 (1992), 115-120; traduzido para o inglês em Siberian Math. J. 33 (1992), 850-855.

[42] A. Pełczyński, Linear extensions, linear averagings, and their applications to linear topological classification of spaces of continuous functions Dissertationes Math.(Rozprawy Mat.) 58, 1968.

[43] A. Pełczyński, Z. Semadeni, Spaces of continuous functions (III). Studia Math. 18 (1959), $211-222$.

[44] H. P. Rosenthal, The Banach space $C(K)$. Handbook of the geometry of Banach spaces, Vol. 2, 1547-1602, North-Holland, Amsterdam, 2003.

[45] W. Rudin, Real and Complex Analysis. Third Edition, McGraw-Hill International Editions, 1987.

[46] C. Samuel, Sur la reproductibilite des espaces $l_{p}$. Math. Scand. 45 (1979), 103-117.

[47] Z. Semadeni, Banach Spaces of Continuous Functions Vol. I. Monografie Matematyczne, Tom 55. Warsaw, PWN-Polish Scientinfic Publishers, Warsaw, 1971.

[48] I. Singer, Best approximation in Normed Linear Spaces by Elements of Linear Subspaces. Die Grundlehren der Mathematischen Wissenschaften in Einzeldarstellungen vol. 171, SpringerVerlag, Berlin Heidelberg New-York, 1970.

[49] I. Singer, Linear functionals on the space of continuous mappings of a compact Hausdorff space into a Banach space. (Russian) Rev. Roum. Math. Pures Appl. 2 (1957), 301-315.

[50] M. H. Stone, Applications of the theory of Boolean rings to general topology. Trans. Amer. Math. Soc. 41 (1937), 375-481. 